NBER WORKING PAPER SERIES

\title{
AUGMENTING MARKETS WITH MECHANISMS
}

\author{
Samuel Antill \\ Darrell Duffie \\ Working Paper 24146 \\ http://www.nber.org/papers/w24146 \\ NATIONAL BUREAU OF ECONOMIC RESEARCH \\ 1050 Massachusetts Avenue \\ Cambridge, MA 02138 \\ December 2017
}

We are grateful for expert research assistance from $\mathrm{Yu} \mathrm{Wu}$, for very helpful conversations with Bruno Biais, Piotr Dworczak, Romans Pancs, and Haoxiang Zhu, for useful feedback from Stanford faculty attending a preliminary presentation of this work on December 8, 2017, for discussions of this paper by Kerry Back at the NBER Asset Pricing Conference, Anton Tsoy at the 2018 Western Finance Association Meeting, and Yunzhi $\mathrm{Hu}$ at the NSF/CEME Decentralization Conference, as well as commenters at the Penn Market Design Conference, the Erasmus Liquidity Conference, the 2018 North American Summer Meeting of the Econometric Society and seminar presentations at ITAM, Stanford University, NYU, Harvard University, Johns Hopkins University, Goethe University, Cambridge University, the University of Chicago, and MIT. We are also grateful to anonymous referees for very helpful expositional suggestions. This material is based upon work supported by the National Science Foundation Graduate Research Fellowship under Grant No. DGE-114747. The views expressed herein are those of the authors and do not necessarily reflect the views of the National Bureau of Economic Research.

NBER working papers are circulated for discussion and comment purposes. They have not been peerreviewed or been subject to the review by the NBER Board of Directors that accompanies official NBER publications.

(C) 2017 by Samuel Antill and Darrell Duffie. All rights reserved. Short sections of text, not to exceed two paragraphs, may be quoted without explicit permission provided that full credit, including $\odot$ notice, is given to the source. 
Augmenting Markets with Mechanisms

Samuel Antill and Darrell Duffie

NBER Working Paper No. 24146

December 2017, Revised June 2020

JEL No. D47,D82,G14

\section{$\underline{\text { ABSTRACT }}$}

We explain how the common practice of size-discovery trade detracts from overall financial market efficiency. At each of a series of size-discovery sessions, traders report their desired trades, generating allocations of the asset and cash that rely on the most recent exchange price. Traders can thus mitigate exchange price impacts by waiting for size-discovery sessions. This waiting causes socially costly delays in the rebalancing of asset positions across traders. As the frequency of size-discovery sessions is increased, exchange market depth is further lowered by the traders' reduced incentive to bid aggressively on the exchange, further delaying the rebalancing of positions, and more than offsetting the gains from trade that occur at each of the size-discovery sessions.

Samuel Antill

Graduate School of Business

Stanford University

655 Knight Way

Stanford, CA 94305-7298

santill@stanford.edu

Darrell Duffie

Graduate School of Business

Stanford University

Stanford, CA 94305-7298

and NBER

duffie@stanford.edu 


\section{Introduction}

In financial markets, investors with large trading interests recognize that their trades can move the market-clearing price against themselves. These investors strategically avoid price-impact costs by executing large orders slowly. The result is that, in equilibrium, the asset is reallocated across traders more gradually than is socially optimal. This concern is exacerbated, under post-crisis regulations, by the higher costs for intermediary dealer banks of absorbing large customer orders onto their own balance sheets. Trade venue operators have introduced sizediscovery sessions that allow market participants to lower their price-impact costs by using trade protocols such as workups and dark pools that set the terms of trade based on the fixed price set on the exchange. We show that, at least in our model setting, overall allocative efficiency is reduced by augmenting price-discovery exchange markets with size discovery. This is true even for size-discovery mechanism designs that efficiently reallocate the asset at every session.

It is already well understood from the work of Vayanos (1999), Rostek and Weretka (2015), and Du and Zhu (2017) that traders bid less aggressively in a financial market in order to strategically lower their price impacts, causing socially costly delays in rebalancing positions across traders. ${ }^{1}$ In our model, price discovery occurs on an exchange that is modeled as a sequential-double-auction market, along the lines of Du and Zhu (2017). Each auction is a demand-function submission game, in the sense of Wilson (1979) and Klemperer and Meyer (1989).

We examine the welfare implications of augmenting exchange trade with size-discovery trade. At each of a sequence of size-discovery sessions, as in common practice, the terms of trade are based on the most recent exchange price. Because this price is frozen for the purpose of sizediscovery trade, price impacts are avoided. As an example, we show that size discovery can be based on a simple dark-pool mechanism that uses the most recent exchange price. We also provide a non-linear strategy-proof mechanism design for size discovery. ${ }^{2}$

At each size-discovery session, traders are induced by the mechanism design to truthfully report their excess inventories of the asset to the platform operator, which then allocates transfers of cash and the asset. In equilibrium, each session is ex-post individually rational and incentive compatible, budget balanced, and reallocates the asset perfectly efficiently among traders. This seeming contradiction of Myerson and Satterthwaite (1983) is possible because of the information available to the size-discovery platform operator through the prior equilibrium

\footnotetext{
${ }^{1}$ Sannikov and Skrzypacz (2016) study a similar setting with heterogeneous traders. They also consider mechanism design, but solely as an analytical device to solve for the equilibrium of a conditional double-auction model.

${ }^{2}$ Specifically, our results hold for a class of non-linear mechanisms that contains the Vickrey-Clarke-Groves mechanism associated with the equilibrium value functions.
} 
exchange price. As the mean frequency of size-discovery sessions is increased, traders reduce their exchange order submissions, relying more on upcoming size-discovery sessions because of their lower price-impact costs. Exchange market depth is therefore reduced, further increasing the incentive to shade exchange order submissions. Traders also shade their exchange order submissions in order to reduce the impact of their exchange trades on the expected price that will be used to set the terms of trade at the next size-discovery session. Overall, the significant expected gains from trade that occur at each size-discovery session are more than offset by the effect of reduced exchange trading. Indeed, every trader is made worse off, ex ante, by the presence of size discovery. If, however, a size-discovery venue is available, traders will participate whenever a size-discovery session is held.

Even if the size-discovery mechanism designer has enough information to avoid reliance on exchange prices to set the terms of trade, we show that welfare cannot be improved by adding size discovery, except for a size-discovery session that is run before the exchange market opens, as shown by Duffie and Zhu (2017), who analyzed workup, a form of size discovery that is heavily used in dealer-dominated markets, such as those for treasuries and swaps. Duffie and Zhu (2017) also showed that workup is not a fully efficient form of size discovery because traders under-report the sizes of their positions (or equivalently, under-submit trade requests), relative to socially optimal order submissions, due to a winner's-curse effect. As a mechanism design, the workup protocol places strong restrictions on the allowable forms of messages and transfers. Our modeled size-discovery trade protocols are based on mechanism designs that reallocate the asset with maximal efficiency. After each size-discovery session, however, traders' asset inventories are hit by new supply and demand shocks over time that cause a desire for further rebalancing.

In our model, augmenting the exchange market with size-discovery sessions has no social value, because the allocative benefits of size-discovery sessions are more than fully offset by a corresponding reduction in gains from trade on the exchange market. While one might imagine that this relatively discouraging result is caused by a size-discovery mechanism design that is "too efficient," we show that overall allocative efficiency is not helped by impairing the efficiency of the size-discovery protocol (at least within a given class of mechanisms) in order to better support exchange market depth and trade volumes.

For tractability, we assume that traders have symmetric quadratic inventory holding costs and that size-discovery sessions are held at Poisson arrival times. Despite this narrow model parameterization, the underlying intuition for our welfare result seems relatively general. It is natural that size discovery increases the incentive of individual traders to delay socially beneficial exchange trading. Traders wait for size-discovery sessions in order to mitigate their price-impact costs. But a price impact is merely a wealth transfer, not a welfare cost. Thus, 
size discovery represents a coordination failure. Venue operators offer size discovery because of its popularity. But this popularity is based on private gains of price-impact avoidance that do not contribute to social gains. For example, whatever a buyer loses through price impact is gained by sellers. At the same time, size discovery detracts from a public-good externality, exchange market depth.

Although our results could be useful to regulators who are considering whether to limit or even prohibit size-discovery trading, this paper does not take a normative approach to overall market design. We are examining the social efficiency of market designs that are popular in practice, namely price-discovery exchange markets augmented by size-discovery sessions that set terms of trade based on exchange prices. We do not rule out improvements in overall market design that might be achieved by replacing exchange markets with some alternative approach to trade. We take the presence of exchange markets as given. We also do not consider the augmentation of exchange markets with alternatives to size discovery, perhaps based on mechanism designs that make use of multi-period price and trade data. We merely characterize the costs associated with the common practice of point-in-time size-discovery sessions, at least insofar as it is possible for us to do this with a tractable theoretical model.

Section 2 offers some background on size-discovery practice and a summary of prior related literature. Section 3 contains our basic model and main results. Section 4 offers some additional equilibrium properties of the model related to welfare, the perfect Bayes property, robustness to some alternative preference specifications, and ex-post optimality.

Finally, Section 5 offers a discussion of some additional market-design and policy implications. Here, we consider the potential of a purposeful reduction in the allocative efficiency of size-discovery sessions, with the goal of improving exchange market depth and gains from trade. At least in the setting that we consider, this does not help overall market efficiency. We examine conditions under which eliminating the exchange market and relying only on size discovery alone can improve efficiency. We also consider the competing incentives of exchange operators and size-discovery operators, as well as the coordination failure associated with the lack of incentive of size-discovery operators to consider the impact of their platforms on the depth of price-discovery exchange markets. We raise the potential for policy intervention, including the implications of the recent European Union "double-cap" rules on dark-trade venues.

\section{Background}

The potential harm to the exchange price-formation process caused by size-discovery venues has been an issue of debate among practitioners and policy makers, and is also a point of contention 
in academic research. ${ }^{3}$ In January 2018, the European Union ${ }^{4}$ added rules associated with the Markets in Financial Instruments Directive II (MiFiD II) that place a cap on the volume of trade transacted in dark pools, in order to "not unduly harm price formation." This "double cap" effectively restricts aggregate dark-pool volume to $8 \%$ of total trade volume in affected instruments, and the fraction of trade on any dark pool to $4 \%$ of total volume. ${ }^{5}$ Similar caps or other restrictions on dark-pool trading have been implemented by regulations in Canada and Australia. However, regulators have expressed concern that the effectiveness of this rule may be reduced by exemptions for systematic internalizers (another form of size discovery) and for large trades. ${ }^{6}$ Indeed, Johann, Putnins, Sagade, and Westheide (2019) find that dark-pool bans have simply diverted trade from dark pools to "quasi-dark" trading mechanisms, and have caused a relatively low amount of trade volume to return to exchange markets. They find a negligible impact of dark-pool caps on market liquidity and short-term price efficiency. European Securities and Markets Authority (2020) found that the double volume cap (DVC) limits on dark trading in Europe have led to an increase in periodic-auction trading, which was intended to avoid the DVC limits. Similar findings were reported by French and German official studies. ${ }^{7}$

The most common forms of size discovery used in current market practice are workups, matching sessions, and block-crossing dark pools. As of late 2017, according to Rosenblatt Securities, dark pools account for about $15 \%$ of U.S. equity trading volume. ${ }^{8}$ In the market for U.S. Treasury securities, workup is heavily used on the two dominant inter-dealer electronic trade platforms, BrokerTec and eSpeed. Fleming and Nguyen (2015) estimate that workup accounts for $43 \%$ to $56 \%$ of total trading volume on the largest Treasuries trade platform, BrokerTec. Once a trade is executed on BrokerTec's limit-order book at some price, a workup session can be opened for potential additional trading at the same "frozen" price. The original buyer and seller and other platform participants may submit additional buy and sell orders

\footnotetext{
${ }^{3}$ See, for example, CFA Institute (2012) and the discussions of Zhu (2014) and Ye (2016).

${ }^{4}$ The exact implementation dates of each piece of MiFiD II vary, see https://www.fca.org.uk/markets/ mifid-ii.

${ }^{5}$ Article 5 restricts the waivers of Article 4 such that "the percentage of trading in a financial instrument carried out on a trading venue under those waivers shall be limited to $4 \%$ of the total volume of trading in that financial instrument on all trading venues across the Union over the previous 12 months," and "overall Union trading in a financial instrument carried out under those waivers shall be limited to $8 \%$ of the total volume of trading in that financial instrument on all trading venues across the Union over the previous 12 months." See http://eur-lex.europa.eu/legal-content/EN/TXT/?uri=uriserv:OJ.L_.2014.173.01. 0084.01.ENG for the text of Regulation (EU) No 600/2014.

${ }^{6}$ As of June 2018, systemic internalizers account for $30 \%$ to $40 \%$ of total market share based on the French market. https://www.ft.com/content/cca902f4-70a1-11e8-92d3-6c13e5c92914?

${ }^{7}$ See Association Française des Marchés Financiers (2019) and German Ministry of Finance (2019). These studies seem to view periodic auctions as failing to contribute to price formation.

${ }^{8}$ See "Let There be Light, Rosenblatt's Monthly Dark Liquidity Tracker," September 2017, at http:// rblt. com/letThereBeLight . aspx?year=2017.
} 
that are executed by time priority at this workup price. Trade on the central-limit-order book is meanwhile suspended. ${ }^{9}$

Matching sessions are a feature of some electronic platforms for trading corporate bonds ${ }^{10}$ and credit default swaps (CDS). The markets for corporate bonds and CDS are distinguished by much lower trade frequency than those for treasuries and equities. Matching sessions, correspondingly, are less frequent and of longer duration. A distinctive feature of matching sessions is that the fixed price is typically chosen by the platform operator. ${ }^{11}$ Collin-Dufresne, Junge, and Trolle (2020) find that matching sessions and workups account for $71.3 \%$ of trade volume for the most popular CDS index product, known as CDX.NA.IG.5yr, a composite of 5-year CDS referencing 125 investment-grade firms, and $73.5 \%$ of trade volume for the corresponding high-yield index product.

Trade platforms for interest-rate swaps also commonly incorporate workup or matchingsession mechanisms, as described by BGC (2015), GFI (2015), Tradeweb (2014), and Tradition (2015). The importance of workup for the interest-rate swap market is discussed by Wholesale Markets Brokers' Association (2012) and Giancarlo (2015).

Empirical evidence regarding the impact on exchange market performance of size-discovery trade is mixed, and limited to equity markets. Size discovery is used far more heavily in bond and swap markets. Degryse, De Jong, and van Kervel (2015) examine trading in Dutch equities across lit (exchange) and unlit trading venues, finding that a one-standard-deviation increase in dark trading activity for a particular stock reduces their metric of lit market depth in that stock by 5.5\%. Nimalendran and Ray (2014) also find that dark trading is associated with greater price impact in lit markets. Hatheway, Kwan, and Zheng (2017) add to the evidence that dark venues harm exchange market liquidity. Using a natural experiment induced by an SEC rule change, however, Farley, Kelley, and Puckett (2017) find no effect of dark trading on exchange market depth. In these studies, dark trading includes not only size-discovery trade, but also other forms of trade that do not have pre-trade price transparency, or that involve hidden trades such as "iceberg" orders. Buti, Rindi, and Werner (2011) estimate that dark pools can actually improve exchange inside-quote depth. ${ }^{12}$ The SEC's Division of Trading and

\footnotetext{
${ }^{9}$ For more details on BrokerTec's workup protocol, see Fleming and Nguyen (2015), Fleming, Schaumburg, and Yang (2015), and Schaumburg and Yang (2016). Liu, Wang, and Wu (2015) provide additional evidence on workups in the GovPX dataset, which focuses on off-the-run Treasury securities.

${ }^{10}$ According to SIFMA (2016), matching sessions are provided by Codestreet Dealer Pool (pending release), Electronifie, GFI, Latium (operated by GFI Group), ICAP ISAM (pending release), ITG Posit FI, Liquidity Finance, and Tru Mid.

${ }^{11}$ GFI, for example, chooses a matching-session price that is based, according to SIFMA (2016), on "GFI's own data (input from the internal feeds), TRACE data, and input from traders." On the CDS index trade platform operated by GFI, the matching price "shall be determined by the Company [GFI] in its discretion, but shall be between the best bid and best offer for such Swap that resides on the Order Book."

${ }^{12}$ The inside-quote depth is the number of shares available at the best bid and best offer on the limit order
} 
Markets (2013) provide a more detailed summary of empirical evidence regarding the impact of dark trade on exchange markets.

In prior work on mechanism design in dynamic settings, Bergemann and Välimäki (2010) show that a generalization of the Vickrey-Clarke-Groves pivot mechanism can implement efficient allocations in dynamic settings with independent private values. Similarly, Athey and Segal (2013) and Pavan, Segal, and Toikka (2014) study optimal mechanism designs in dynamic settings with independent types. As opposed to this prior research, we focus on a market setting in which agents cannot be obliged ${ }^{13}$ to participate in mechanism sessions or to abstain from trading on existing exchanges.

Dworczak (2020) precedes this paper in considering a mechanism design problem in which the designer cannot prevent agents from participating in a separate market. Beyond that likeness of perspective, the problems addressed by our respective models are quite different. Ollár, Rostek, and Yoon (2017) address a design problem associated with double-auction markets, but focus instead on information revelation within the market, rather than an augmentation of the double-auction market with mechanism-based sessions. Du and Zhu (2017) and Budish, Cramton, and Shim (2015) consider the optimal frequency of batch auctions as a market-design approach. Pancs (2014) analyzed the implications of workup for its ability to mitigate frontrunning. 14

\section{Augmenting price discovery with size discovery}

This section presents the main model and results. We consider a stochastic market game consisting of a continually operating "price-discovery" exchange market that is augmented with randomly timed size-discovery sessions. On the exchange, modeled as a sequential doubleauction market, investors strategically avoid price impact, causing a socially inefficient delay in the rebalancing of asset positions across traders. The social costs of the strategic avoidance of exchange price impact are well covered by the results of Vayanos (1999), Rostek and Weretka

\footnotetext{
book.

${ }^{13}$ Specifically, we always impose an ex-post participation condition that, at every mechanism session, all traders prefer participation to the outside option of not entering this mechanism and trading in a doubleauction market until the next mechanism. In contrast, Pavan, Segal, and Toikka (2014) force agents to commit at time zero to participate in all future mechanisms (or post an arbitrarily large bond to be forfeited in the event of exit), and Bergemann and Välimäki (2010) force agents to forgo all future mechanism participation in order to sit out one mechanism event. Athey and Segal (2013) provide conditions under which efficient allocations can be reached without participation constraints, but only if agents are arbitrarily patient relative to the most extreme (finite) realization of uncertainty.

${ }^{14}$ The seller in Panc's model has private information about the size of his or her desired trade. The buyer is either a "front-runner" or a dealer. If the seller cannot sell the entire large position in workup, he would need to liquidate the remainder by relying on an exogenously given outside demand curve.
} 
(2015), Du and Zhu (2017), and Duffie and Zhu (2017). Our main result is that the popular practice of augmenting exchange markets with size discovery does not mitigate these social costs, at least in our model setting, even though all traders wish to use size discovery if it is available and even though each size-discovery session achieves a socially efficient reallocation of the asset.

The continuous-time presentation of our results is chosen for its expositional simplicity. Appendix F offers a discrete-time analogue. Although the discrete-time setting generates messier looking results, it allows us to demonstrate a standard equilibrium robustness property, Perfect Bayes. The equilibrium behavior of the discrete-time model converges to that of the continuoustime model as the length of a time period shrinks to zero.

\subsection{Preliminaries}

We fix a probability space $(\Omega, \mathcal{F}, \mathbb{P})$, the time domain $[0, \infty)$, and an information filtration $\mathbb{F}=\left\{\mathcal{F}_{t}: t \geq 0\right\}$ of sub- $\sigma$-algebras of $\mathcal{F}$ satisfying the usual conditions. ${ }^{15}$ The market is populated by $n \geq 3$ risk-neutral traders exchanging a divisible asset. The asset payoff $\pi$ is a finite-variance random variable with mean $v$. The payoff $\pi$ is revealed publicly and paid to traders at a random time $\mathcal{T}$ that is exponentially distributed with parameter $r$. Thus $\mathbb{E}(\mathcal{T})=1 / r$. There is no further incentive to trade once the asset dividend is paid at time $\mathcal{T}$, which is therefore the end time of the model.

For purposes of submitting demands to the exchange, trader $i$ has information given by a sub-filtration $\mathbb{F}^{i}=\left\{\mathcal{F}_{t}^{i}: t \geq 0\right\}$ of $\mathbb{F}$. The traders have symmetric information about the asset payoff. Specifically, the conditional distribution of $\pi$ given $\mathcal{F}_{t}$ is constant until the payoff time $\mathcal{T}$, so that no trader ever learns anything about $\pi$ until the market ends. Traders may, however, have asymmetric information about their respective asset positions at each time. Price fluctuations are thus driven only by rebalancing demands, and not by learning about ultimate asset payoffs. This informational setting is more relevant for markets such as those for stock index products, major currencies, and fixed income products such as swaps and government bonds. For example, there is typically symmetric information about the payoff of a U.S. Treasury bill, but the price of a U.S. Treasury bill fluctuates randomly over time, partly caused by shocks to the allocation of the bills across market participants.

15 Given our probability space, the "usual conditions" on the filtration are precisely defined in, for example, Protter (2005). These conditions are that the filtration is complete, increasing, and right-continuous. 


\subsection{Trader excess inventories}

The "excess inventory" of the asset held by trader $i$ at time $t$ is denoted $z_{t}^{i}$. A trader's excess inventory in this model can be viewed as the trader's total asset position net of the trader's desired asset position, so that all traders would ideally wish to achieve an excess inventory of zero. The respective initial excess inventories of the asset for the $n$ traders are given by a list $z_{0}=\left(z_{0}^{1}, z_{0}^{2}, \ldots, z_{0}^{n}\right)$ of finite-variance random variables, with $z_{0}^{i}$ measurable with respect to $\mathcal{F}_{0}^{i}$. Each trader $i$ can use the exchange market and size-discovery platform to change their excess inventory $z_{t}^{i}$ by trading, as we now describe in detail.

\subsection{The exchange market}

In the continually operating exchange market, trader $i$ submits an $\mathbb{F}^{i}$-progressively measurable ${ }^{16}$ demand function $D^{i}: \Omega \times \mathbb{R}_{+} \times \mathbb{R} \rightarrow \mathbb{R}$. Thus, in state $\omega$ at time $t$, if the outcome of the auction price is $p$, trader $i$ would buy the asset at the quantity "flow" rate $D^{i}(\omega, t, p)$. Given an exchange market price process $\phi$, the total asset purchase of trader $i$ is thus $\int_{0}^{\mathcal{T}} D^{i}\left(t, \phi_{t}\right) d t$, assuming that this integral exists. (As is typical for notational simplification, we suppress the state $\omega$ from the expression.) A demand function $D^{i}$ for trader $i$ is said to be admissible if, for each squareintegrable $^{17}$ price process $\phi$, the resulting demand process $\left\{D^{i}\left(t, \phi_{t}\right): t \geq 0\right\}$ is also square integrable, thus implying that the total expected exchange purchase cost $\mathbb{E}\left[\int_{0}^{\mathcal{T}} D^{i}\left(t, \phi_{t}\right) \phi_{t} d t\right]$ is well defined.

We only consider equilibria in which demand functions are of the form

$$
D^{i}(\omega, t, p)=a+b p+c z_{t}^{i}(\omega)
$$

for constants $a, b<0$, and $c$ that do not depend on the trader $i$, state $\omega$, or time $t$, and where $z_{t}^{i}$ is the excess inventory of trader $i$ at time $t$, as explained in Section 3.2. To be clear, the traders are not restricted to demand functions that take the simple form (1), but we will show that in equilibrium each trader optimally chooses a demand function that is implemented by a function of this affine form (1) if he or she assumes that the other traders do so. We focus on equilibria that are symmetric, in the sense that the constants $a, b$, and $c$ are the same for each trader $i$. There may exist asymmetric linear equilibria, or nonlinear equilibria. For tractability we focus exclusively on "symmetric affine equilibria," those with demand functions of the form (1), with the same coefficients. Because the excess-inventory process $z^{i}$ and the price process $\phi$

\footnotetext{
${ }^{16}$ A function $X: \Omega \times \mathbb{R}_{+} \times \mathbb{R} \rightarrow \mathbb{R}$ is progressively measurable with respect to $\mathbb{F}^{i}$ if it is measurable on the product space $\left(\Omega \times \mathbb{R}_{+} \times \mathbb{R}, \mathcal{F} \otimes \mathcal{B}\left(\mathbb{R}_{+}\right) \otimes \mathcal{B}(\mathbb{R})\right)$, where $\mathcal{B}(\cdot)$ denotes the Borel $\sigma$-algebra, and if, for any $\mathbb{F}^{i}$-adapted measurable process $\phi$, the process $(\omega, t) \mapsto X(\omega, t, \phi(\omega, t))$ is adapted.

17 A process $u$ is square integrable if $u$ is progressively measurable and $\mathbb{E}\left(\int_{0}^{\mathcal{T}} u_{t}^{2} d t\right)<\infty$.
} 
are naturally assumed to be observed by trader $i$, the demand function (1) is $\mathbb{F}^{i}$-progressively measurable, as required. Furthermore, we will show that, in equilibrium, $z^{i}$ and $\phi$ are squareintegrable processes, so that any demand function $D^{i}$ of the form (1) is also square integrable, thus admissible.

The exchange trade protocol is a double auction. That is, at time $t$, trades are executed at a market-clearing price $\phi_{t}$ satisfying $\sum_{i} D^{i}\left(t, \phi_{t}\right)=0$, if such a price exists. For the special case (1) of symmetric affine demand functions, the dependence of the unique clearing price on any given trader's demand is characterized by the following lemma.

Lemma 1. Fix a trader $j$ and time $t$. Suppose the affine demand function (1) is submitted by every trader $i \neq j$. For any candidate demand $d \in \mathbb{R}$ by trader $j$, there is a unique price $p$ satisfying $d+\sum_{i \neq j}\left(a+b p+c z_{t}^{i}\right)=0$. This clearing price is

$$
p=\Phi_{(a, b, c)}\left(d ; Z_{t}^{-j}\right) \equiv \frac{-1}{b(n-1)}\left(d+(n-1) a+c Z_{t}^{-j}\right),
$$

where $Z_{t}^{-j} \equiv \sum_{i \neq j} z_{t}^{i}$

Thus, for any non-degenerate affine demand function used by $n-1$ of the traders, there is a unique market-clearing price corresponding to each quantity chosen by the remaining trader. The proof is a straightforward calculation. ${ }^{18}$ Under the conditions of Lemma 1, from the strategic viewpoint of trader $i$, it is therefore equivalent whether to submit a demand function $D^{i}$ which, at each state $\omega$ and time $t$, is a demand schedule $p \mapsto D^{i}(\omega, t, p)$, or alternatively to take the affine demand functions $\left\{D^{j}: j \neq i\right\}$ of the other traders as given and to submit a demand process $\hat{D}^{i}: \Omega \times \mathbb{R} \rightarrow \mathbb{R}$ that executes the quantity $\hat{D}_{t}^{i}$ at the price $\Phi_{(a, b, c)}\left(\hat{D}_{t}^{i} ; Z_{t}^{-i}\right)$. An equilibrium consistency condition is that these are outcome-equivalent, in that, for all $(\omega, t)$,

$$
\hat{D}^{i}(\omega, t)=D^{i}\left(\omega, t, \Phi_{(a, b, c)}\left(\hat{D}^{i}(\omega, t) ; Z^{-i}(\omega, t)\right)\right)=a+b \Phi_{(a, b, c)}\left(\hat{D}^{i}(\omega, t) ; Z^{-i}(\omega, t)\right)+c z^{i}(\omega, t) .
$$

Trader $i$ submits exchange demands strategically, bearing in mind the costly impact on the clearing price $\Phi_{(a, b, c)}\left(d ; Z_{t}^{-i}\right)$ of his or her demand $d$. But this price impact is merely a wealth transfer among traders that has no direct social cost. In particular, it is not socially efficient for traders to internalize their price-impact costs, as shown by Vayanos (1999), Rostek and Weretka (2015), and Du and Zhu (2017).

\footnotetext{
18 Because $b \neq 0$, the following statements are equivalent: (i) $d+\sum_{i \neq j}\left(a+b p+c z_{t}^{i}\right)=0$, (ii) $-b(n-1) p=$ $d+(n-1) a+c Z_{t}^{-j}$, and (iii) $p=-\left(d+(n-1) a+c Z_{t}^{-j}\right) /[b(n-1)]$.
} 


\subsection{Excess inventory costs}

As a motive for trade, the excess asset inventory of trader $i$ is randomly shocked over time with additional units of the asset. The cumulative shock to the excess inventory of trader $i$ by time $t$ is $H_{t}^{i}$, for some finite-variance $\mathbb{F}^{i}$-adapted Lévy process $H^{i}$ that is a martingale with respect to $\mathbb{F}$ and thus with respect to the information filtration $\mathbb{F}^{i}$ of trader $i$. A simple example is a Brownian motion with zero drift. The defining property of a Lévy process is that it has iid increments over any set of equally long disjoint time intervals. Without loss of generality, we take $H_{0}^{i}=0$. The excess inventory shock processes $H=\left(H^{1}, \ldots, H^{n}\right)$ need not be independent, nor identically distributed, across traders, but we assume that $H, \mathcal{T}, \pi$, and $z_{0}$ are mutually independent. We assume that $\sum_{i=1}^{n} H^{i}$ is also a Lévy process with respect to $\mathbb{F}$. We let $Z_{t} \equiv \sum_{i} z_{0}^{i}+H_{t}^{i}$ denote the aggregate excess inventory.

Traders suffer costs associated with unwanted levels of inventory, whether too large or too small. One may think in terms of a market maker that is attempting to run a matched book of positions, but which may accept customer positions over time that shock its inventory. The market maker may then trade so as to lay off excess inventories with other market makers.

The market practitioners Almgren and Chriss (2001) proposed a simple model of excess inventory costs for financial firms that is now popular among other practitioners and also in the related academic research literature, by which the rate of excess inventory cost to trader $i$ at time $t$ is $\gamma\left(z_{t}^{i}\right)^{2}$, for some fixed coefficient $\gamma>0$. With this cost model, trader $i$ bears an expected total cost of future undesired inventory of $\mathbb{E}\left[\int_{0}^{\mathcal{T}} \gamma\left(z_{t}^{i}\right)^{2} d t\right]$. The first-best (socially optimal) allocation of the asset is that minimizing the total of the traders' excess inventory costs, which is the equal allocation given by $z_{t}^{i}=\bar{Z}_{t}$, where $\bar{Z}_{t} \equiv Z_{t} / n$ is the average excess inventory. The equal allocation remains socially optimal if the quadratic excess inventory cost function $z \mapsto \gamma z^{2}$ is replaced with any convex even function, however we have been able to obtain clearly stated results only for the quadratic special case.

Although financial firms have no "psychic" aversion to risk, broker-dealers and asset-management firms do have extra costs for holding excess inventory in illiquid or risky assets. These costs can be related to regulatory capital requirements, collateral requirements, financing costs, agency costs associated with a lack of transparency of the quality of the asset to higher-level firm managers or clients, as well as the expected cost of being forced to suddenly raise liquidity by quickly disposing of remaining inventory into an illiquid market. Although it has not been given a structural micro-foundation, the quadratic holding-cost model that we use is common in dynamic market-design models, including those of Vives (2011), Rostek and Weretka (2012), Du and Zhu (2017), and Sannikov and Skrzypacz (2016). 


\subsection{Size discovery}

Size-discovery sessions are held at each of the event times $\tau_{1}, \tau_{2}, \ldots$ of a Poisson process $N$ with mean arrival rate $\lambda>0$. The session-timing process $N$ is independent of the other primitive random variables, $\left\{H, \mathcal{T}, \pi, z_{0}\right\}$. The $k$-th size-discovery session is announced at time $\tau_{k}$ after exchange demand submissions have been made and the exchange price $\phi\left(\tau_{k}\right)$ has been determined. That is, at time $\tau_{k}$, the traders' exchange demands are submitted and the exchange price is determined before the traders are aware ${ }^{19}$ that there will be a size-discovery session. Once the size-discovery session is announced, trader $i$ either declines to participate in the session or provides an excess-inventory report to the size-discovery platform. That is, the strategy of trader $i$ at the $k$-th session has an outcome in the space $\mathcal{M}=\mathbb{R} \cup\{\nu\}$, where the choice $\nu$ denotes non-participation and any choice in $\mathbb{R}$ is a participating excess-inventory report. The information filtration used by trader $i$ for making size-discovery decisions is that generated by both $^{20} \mathbb{F}^{i}$ and $N$. Traders are free to misreport their privately observed inventories. A reported excess inventory is restricted to having finite variance. A truthful excess-inventory report from trader $i$ is given by $\mu_{k}^{i}=z^{i}\left(\tau_{k}\right)$. If any trader $i$ declines to participate, in that $\mu_{k}^{i}=\nu$, the size-discovery platform does nothing - there are no transfers of cash or assets to any trader, and the exchange market continues operating until the next size-discovery session is announced, and so on. This formulation implies that our equilibrium condition of the optimality of truthful reporting for the size-discovery strategy $\mu^{i}$ of trader $i$ also includes the individual rationality (IR) condition that trader $i$ is actually willing to participate in excess-inventory reporting at all size-discovery sessions. The size-discovery allocations are based on mechanism designs that we describe in this section, and whose properties are developed in Appendix A.

In practice, the timing of size-discovery sessions varies significantly across markets. For example, workup sessions in BrokerTec's market for Treasury securities occur at an average frequency of about 600 times a day for the 2-year note, and about 1400 times a day for the 5year note, according to statistics provided by Fleming and Nguyen (2015). These size-discovery sessions account for approximately half of all trade volume in Treasury securities on BrokerTec, which is by far the largest trade platform for U.S. Treasurys, accounting for an average of over $\$ 30$ billion in daily transactions for each of the 2-year, 5-year, and 10-year on-the-run Treasury notes. Consistent with our model, BrokerTec workup sessions are held at randomly

\footnotetext{
${ }^{19}$ Specifically, for all $t, \mathcal{F}_{t}^{i}$ and $\left\{N_{u}-N_{t}: u \geq t\right\}$ are independent, but the left-continuous process $\hat{N}$, defined by $\hat{N}_{t}=\lim _{s \uparrow t} N_{s}$, is adapted to $\mathbb{F}^{i}$. This means that $\mathbb{F}^{i}$ does not satisfy the usual condition of right continuity, but this does not matter for our analysis.

${ }^{20}$ That is, the choice $\mu_{k}^{i}: \Omega \rightarrow \mathcal{M}$ of trader $i$ at the $k$-th session time is required to be $\overline{\mathcal{F}}_{\tau_{k}}^{i}$-measurable, where $\overline{\mathcal{F}}_{t}^{i}$ is the completion of the $\sigma$-algebra generated by $\mathcal{F}_{t}^{i}$ and $\left\{N_{s}: s \leq t\right\}$. Our stochastic integrals are defined with respect to the bigger filtration $\left\{\overline{\mathcal{F}}_{t}^{i}: t \geq 0\right\}$, which satisfies all of the usual conditions, including right-continuity.
} 
spaced times. As opposed to our model, however, the times of BrokerTec workup sessions are not exogenous - they are chosen by market participants. In the corporate bond market, "matching sessions," another form of size discovery, occur with much lower frequency, such as once per week for some bonds. The matching sessions on Electronifie, a corporate bond trade platform, are triggered automatically by an algorithm that depends on the current order book and the unfilled portion of the last order-book trade. Again, this differs from our simplifying assumption that size-discovery reallocation sessions occur at independent exogenously chosen times.

A key feature of size-discovery mechanisms such as dark pools, workups, and matching sessions, is that the size-discovery price terms are "frozen" when the size-discovery session is held. In this way, price impacts are mitigated. ${ }^{21}$

The role of the exchange price $\phi_{t}$ in our mechanism designs is analogous to that for conventional forms of size discovery used in practice, such as workups and dark pools. In a dark pool, as explained by Zhu (2014), the per-unit price is set by protocol to the immediately preceding mid-price in a designated limit-order-book market. In BrokerTec's Treasury-market workup sessions, as explained by Fleming and Nguyen (2015), the frozen price used for workup compensation is fixed at the last trade price in the immediately preceding order-book market operated by the same platform provider. In matching sessions, the frozen price is set based on an estimate of prevailing prices in recent trades. Thus, in dark pools, workup, and other forms of size discovery used in practice, and also in this setting for our model, there is an incentive for traders to bid strategically in the exchange market so as to avoid worsening their expected cash compensation terms in the next size-discovery session, through their impact on exchange prices. As we will show, this additional strategic incentive for shading exchange market bids delays the rebalancing of positions across traders, causing a strict reduction in welfare relative to a market with no size discovery.

A size-discovery mechanism design consists of an asset transfer $Y: \mathcal{M}^{n} \rightarrow \mathbb{R}^{n}$ and a cash transfer $T: \mathcal{M}^{n} \times \mathbb{R} \rightarrow \mathbb{R}^{n}$ that, given the already-determined exchange market price $p$, map a vector $\mu$ of agent choices to a vector $Y(\mu)$ of asset transfers and a vector $T(\mu, p)$ of cash transfers, respectively. In the event of the non-participation choice $\mu^{i}=\nu$ for at least some trader $i$, we take $T(\mu, p)=Y(\mu)=0$.

\footnotetext{
${ }^{21}$ Not all dark pools are designed primarily for the purpose of mitigating price impacts for large orders. Drawing from an industry report by Rosenblatt Securities, Ye (2016) notes that "In May 2015, among the 40 active dark pools operating in the US, there are 5 dark pools in which over $50 \%$ of their Average Daily Volumes are block volume (larger than 10k per trade). Those pools can be regarded as "Institutional dark pools," and they include Liquidnet Negotiated, Barclays Directx, Citi Liquifi, Liquidnet H20, Instinet VWAP Cross, and BIDS Trading." Other objectives of dark pool users include a reduction in the leakage of private information motivating trade, and the avoidance of bid-ask spread costs. Some broker-dealers use their own dark pools to internalize order executions among their clients.
} 
For purely technical reasons, we require $Y$ and $T$ to be measurable, and, when restricted to the domain $\mathbb{R}^{n} \times \mathbb{R}$ that is based on participating excess-inventory reports, $Y$ to be bounded by a function that is Lipschitz, and $T$ to be bounded by a second-order polynomial in $(\mu, p)$. All of the specific size-discovery mechanisms that we consider in this paper satisfy these technical requirements.

We next consider two different mechanism designs for size discovery that achieve truthful reporting and a socially efficient asset reallocation at each size-discovery session. A third sizediscovery mechanism, based on a common design for dark pools, is analyzed in Appendix C.4. All three of these mechanisms assign budget-balanced cash transfers and exactly balanced asset transfers given any possible vector of reports. In equilibrium, traders facing any of these mechanisms find it individually rational to participate and incentive compatible to truthfully report. There are likely to be other mechanism designs with these properties.

\subsubsection{A dark-pool mechanism}

The dark-pool mechanism design $\left(Y, T_{D}\right)$ for size discovery assigns trades that are compensated at a frozen price. The frozen price is given by the last exchange price. Formally, for any $(\mu, p) \in \mathbb{R}^{n} \times \mathbb{R}$, the dark-pool size-discovery mechanism assigns trades and cash payments, respectively, given by

$$
\begin{aligned}
Y^{i}(\mu) & =\frac{\sum_{j=1}^{n} \mu^{j}}{n}-\mu^{i} \\
T_{D}^{i}(\mu, p) & =-p Y^{i}(\mu) .
\end{aligned}
$$

For expositional simplicity, (4) specifies additive rationing. In practice, dark pools commonly specify proportional rationing, by which the heavy side of the market is rationed in proportion to the sizes of individual orders on the heavy side. For example, with proportional rationing, on the event that the total of buy orders exceeds the total quantity $Q$ of sell orders, buyer $i$ is assigned the product of $Q$ and the fraction of the total quantity of buy orders that were submitted by buyer $i$. Appendix C.4 shows that our results are unchanged with proportional rationing. Specifically, we consider a game in which size-discovery participants report a desired trade size, which is proportionally rationed and then cleared at the frozen price given by the last exchange price. We show that the resulting equilibria are allocatively identical to those for the two size-discovery mechanisms considered in this section, linear-rationing dark pools and linear-quadratic size discovery.

Given truthful reporting, the asset transfer (4) generates the efficient post-session excess inventory $\bar{Z}_{t}$ for every trader. Truthful reporting is incentive compatible in the equilibria 
that we consider. Intuitively, when agents take the previously determined exchange price as frozen, thus not internalizing their own price impacts, they trade to an efficient allocation. This idea is familiar from the allocative model of Walras (1877) and the implications of the First Welfare Theorem. The dark-pool mechanism is exactly budget balanced because the total asset reallocation $\sum_{i} Y^{i}(\mu)$ is zero and Walras' Law applies, in that the total cash transfer $\sum_{i} T_{D}^{i}(\mu, p)=0$ is always zero.

\subsubsection{A linear-quadratic size-discovery mechanism}

An alternative mechanism design $\left(Y, T_{Q}\right)$ for size discovery that also induces truthful reporting and a socially efficient allocation is given by the asset transfer, at any $(\mu, p) \in \mathbb{R}^{n} \times \mathbb{R}$, specified by equation (4) and, for any fixed strictly negative constant $\kappa_{0}$, the linear-quadratic cash transfer

$$
T_{Q}^{i}(\mu, p)=p \mu^{i}+\kappa_{0}\left(-n \beta(p)+\sum_{j=1}^{n} \mu^{j}\right)^{2}-p \beta(p)+\frac{p^{2}}{4 \kappa_{0} n^{2}}
$$

where

$$
\beta(p)=\frac{r v}{2 \gamma}+p\left(\frac{-r}{2 \gamma}+\frac{1}{2 \kappa_{0} n^{2}}\right) .
$$

The first term of the cash transfer $T_{Q}^{i}(\mu, p)$ is the product of the frozen exchange price $p$ and the excess-inventory report $\mu^{i}$. When combined with the quadratic second term, truth telling is induced. The constant final term ensures that this design is budget feasible, in that the total payment $\sum_{i} T_{Q}^{i}(\mu, p)$ to the traders is at most zero for any trader reports, and is equal to zero in equilibrium. Appendix A demonstrates these and other properties of this mechanism design, and states a particular choice for $\kappa_{0}$ for which the cash transfers coincide with the VickreyClarke-Groves (VCG) mechanism ${ }^{22}$ transfers, making the mechanism strategy proof. Thus, although more complicated than the dark-pool mechanism, the linear-quadratic mechanism is more robust to noise in trader reporting.

\subsection{Equilibrium}

Given a size-discovery mechanism design $(Y, T)$, a symmetric equilibrium for the associated stochastic game is defined by a collection $(a, b, c)$ of affine demand-function coefficients with the following properties:

1. For each $j$, suppose trader $j$ conjectures that all other traders use the strategy: (i)

\footnotetext{
${ }^{22}$ This well-studied mechanism is based on the work of Vickrey (1961), Clarke (1971), and Groves (1973).
} 
exchange demands specified by the affine demand function with coefficients $(a, b, c)$; (ii) participation in size discovery with truthful excess-inventory reporting. Then the same strategy is optimal for trader $j$.

2. If each trader submits exchange demands specified by the affine demand function with coefficients $(a, b, c)$, then the exchange market clears.

"Individual rationality" means the optimality for each agent of participating in size-discovery and exchange-market trading whenever these protocols are offered, based on all current information. We calculate and verify equilibria by solving each agent's equilibrium stochastic control problem for optimal exchange demands and size-discovery reporting, including participation decisions, as follows.

As we have noted, Lemma 1 implies that, given the affine demand functions of other traders, it is equivalent from the viewpoint of trader $i$ whether to choose a demand function $D^{i}$ : $\Omega \times \mathbb{R}_{+} \times \mathbb{R} \rightarrow \mathbb{R}$ or to choose a square-integrable demand process $D: \Omega \times \mathbb{R}_{+} \rightarrow \mathbb{R}$ that sets the clearing price $\Phi_{(a, b, c)}\left(D_{t} ; Z_{t}^{-i}\right)$. In a symmetric equilibrium with demand coefficients $(a, b, c)$, the problem faced by trader $i$ can thus be reduced to choosing an admissible demand process $D^{i}$ and an admissible sequence $\mu^{i}=\left\{\mu_{1}^{i}, \mu_{2}^{i}, \ldots\right\}$ of size-discovery actions solving the stochastic control problem

$$
\sup _{(D, \mu)} \mathbb{E}\left[J^{i}(D, \mu, 0) \mid \mathcal{F}_{0}^{i}\right]
$$

where, for any time $t_{0}$,

$$
\begin{aligned}
J^{i}\left(D, \mu, t_{0}\right)=z_{\mathcal{T}}^{D, \mu} \pi & -\int_{t_{0}}^{\mathcal{T}}\left[\gamma\left(z_{t}^{D, \mu}\right)^{2}+\Phi_{(a, b, c)}\left(D_{t} ; Z_{t}-z_{t}^{D, \mu}\right) D_{t}\right] d t \\
& +\sum_{\left\{k: t_{0}<\tau_{k}<\mathcal{T}\right\}} T^{i}\left(\left(\mu_{k}, \mu_{k}^{-i}\right), \Phi_{(a, b, c)}\left(D_{\tau_{k}} ; Z_{\tau_{k}}-z_{\tau_{k}}^{D, \mu}\right)\right),
\end{aligned}
$$

subject to

$$
\begin{aligned}
z_{t}^{j} & =z_{0}^{j}+\int_{0}^{t} \hat{D}_{s}^{j} d s+H_{t}^{j}+\sum_{\left\{k: t_{0}<\tau_{k}<t\right\}} Y^{j}\left(\left(\mu_{k}, \mu_{k}^{-i}\right)\right) \\
z_{t}^{D, \mu} & =z_{0}^{i}+\int_{0}^{t} D_{s} d s+H_{t}^{i}+\sum_{\left\{k: t_{0}<\tau_{k}<t\right\}} Y^{i}\left(\left(\mu_{k}, \mu_{k}^{-i}\right)\right),
\end{aligned}
$$

taking $\mu_{k}^{j}=z^{j}\left(\tau_{k}\right)$ and $\hat{D}_{t}^{j}=a+b \Phi_{(a, b, c)}\left(D_{t} ; Z_{t}-z_{t}^{D, \mu}\right)+c z_{t}^{j}$.

First, we consider equilibria for the linear-quadratic size-discovery mechanism $\left(Y, T_{Q}\right)$. An equilibrium with symmetric affine demand functions is characterized by demand-function co- 
efficients $(a, b, c)$ with the property that, for each trader $i$, the stochastic control problem (8) is solved by the demand function $D^{i}$ of (1) and the individual rationality of size-discovery participation with truthful excess-inventory reports $\mu_{k}^{i}=z^{i}\left(\tau_{k}\right)$. The individual rationality of exchange market participation is automatically satisfied because non-participation at any time is outcome-equivalent to the choice of zero demand.

In equilibrium, the continuation value of trader $i$ is shown in Appendix B to be

$$
\begin{aligned}
V^{i}\left(z_{t}^{i}, Z_{t}\right) & =\mathbb{E}\left[J^{i}\left(D^{i}, \mu^{i}, t\right) \mid \mathcal{F}_{t}^{i}\right] \\
& =\theta_{i}(\lambda, b)+v \bar{Z}_{t}-\frac{\gamma}{r} \bar{Z}_{t}^{2}+\left(v-\frac{2 \gamma}{r} \bar{Z}_{t}\right)\left(z_{t}^{i}-\bar{Z}_{t}\right)-K(\lambda, b)\left(z_{t}^{i}-\bar{Z}_{t}\right)^{2},
\end{aligned}
$$

where $\bar{Z}_{t}=Z_{t} / n$ and

$$
\begin{aligned}
K(\lambda, b) & =\frac{\gamma}{r(n-1)}-\frac{\lambda}{2 b(n-1)}, \\
\theta_{i}(\lambda, b) & =\frac{1}{r}\left(\frac{\gamma}{r} \frac{\sigma_{Z}^{2}}{n^{2}}-K(\lambda, b)\left(\frac{\sigma_{Z}^{2}}{n^{2}}+\sigma_{i}^{2}-2 \frac{\rho^{i}}{n}\right)-\frac{2 \gamma}{r} \frac{\rho^{i}}{n}\right),
\end{aligned}
$$

with $\sigma_{Z}^{2}=\operatorname{var}\left(\sum_{i} H_{1}^{i}\right), \sigma_{i}^{2}=\operatorname{var}\left(H_{1}^{i}\right)$, and $\rho^{i}=\operatorname{cov}\left(Z_{1}, H_{1}^{i}\right)$. An explicit solution for the equilibrium coefficient $b$ is provided below.

We will show that the symmetric affine equilibria for the dark-pool size-discovery design $\left(Y, T_{D}\right)$ coincide precisely with those for linear-quadratic size-discovery mechanism design $\left(Y, T_{Q}\right)$. We also find that the maximal mean frequency $\bar{\lambda}$ of size discovery (whether dark-pool or linear-quadratic) is the unique positive solution of the equation

$$
3 \bar{\lambda}+\sqrt{8 \bar{\lambda}(r+\bar{\lambda})}=(n-2) r .
$$

That is, with $\lambda>\bar{\lambda}$, the relatively quick prospect of a size-discovery session would cause so much bid shading on the exchange market that there is actually no market-clearing price. Of course, beyond the lack of existence of exchange market equilibrium, this would also imply that the size-discovery platform loses access to necessary price information.

For $0<\lambda \leq \bar{\lambda}$, we will show that there are exactly two symmetric affine equilibria. The demand function of one of these equilibria has a bigger slope $|b|$ than that of the other. This equilibrium therefore has higher order flow and greater market depth (lower price impact) than the other. The following proposition characterizes these equilibria and calculates the equilibrium associated with higher order flow, which is the more efficient of the two equilibria. Even for this more efficient equilibrium, we show that size discovery makes every trader worse off. 
Proofs of the following two propositions are provided in Appendices B and C, respectively.

Proposition 1. Fix any $\kappa_{0}<0$ and the associated linear-quadratic size-discovery mechanism design $\left(Y, T_{Q}\right)$. If $\lambda>\bar{\lambda}$, there is no equilibrium with demand functions of the symmetric affine form (1). If $0<\lambda \leq \bar{\lambda}$, there exist precisely two such equilibria. If $\lambda=0$, that is, with no size discovery, there is a unique such equilibrium. Each of these equilibria has the following properties.

1. At time $t$, the exchange price is given by

$$
\phi_{t}=P\left(\bar{Z}_{t}\right) \equiv v-\frac{2 \gamma}{r} \bar{Z}_{t}
$$

2. For $\lambda=0$, and for the more efficient of the two equilibrium in the case of $\lambda>0$ (that with larger $|b|$, and producing the higher continuation value for all traders), the traders' value functions are given by (9) and the demand-function coefficients $(a, b, c)$ are given by

$$
\begin{aligned}
a & =-v b \\
b & =\frac{-r^{2}}{8 \gamma}\left(-\frac{3 \lambda}{r}+(n-2)+\sqrt{\left(\frac{\lambda}{r}-(n-2)\right)^{2}-\frac{4 \lambda n}{r}}\right)<0 \\
c & =\frac{2 \gamma}{r} b .
\end{aligned}
$$

3. For $0<\lambda \leq \bar{\lambda}$ and the more efficient of the two equilibria, market depth $|b|$ and the value function $V^{i}$ of each trader $i$ are strictly decreasing in the mean frequency $\lambda$ of sizediscovery sessions. ${ }^{23}$

Proposition 2. For any mean frequency $\lambda$ of size-discovery, the symmetric equilibria in affine demand functions for the linear-rationing dark-pool size-discovery mechanism $\left(Y, T_{D}\right)$ coincide with those for the linear-quadratic size-discovery design $\left(Y, T_{Q}\right)$. That is, for any $\lambda>\bar{\lambda}$, there are no such equilibria, and otherwise the equilibria for $\left(Y, T_{D}\right)$ and $\left(Y, T_{Q}\right)$ have the same demand-function coefficients $(a, b, c)$, the same exchange market price process $\phi$ given by (13), and, for each trader $i$, the same value function $V^{i}$ specified by (9) and excess-inventory process $z^{i}$.

\footnotetext{
${ }^{23}$ That is, for the equilibrium with the larger absolute size of demand function coefficient $|b|$, the equilibrium demand function price coefficient $b_{\lambda}$ is strictly increasing in $\lambda$ on the domain $[0, \bar{\lambda}]$. The pointwise strict monotonicity of the value function $V^{i}$ in $\lambda$ for this equilibrium then follows from the fact that the constant $K\left(\lambda, b_{\lambda}\right)$ of $(10)$ is positive and strictly monotonically increasing in $\lambda$, which implies that $\theta_{i}\left(\lambda, b_{\lambda}\right)$ is also strictly monotonically decreasing in $\lambda$.
} 
Appendix C.4 provides an analogous result for the case of proportional-rationing dark pools, as commonly used in practice, for which the equilibria again have the same demand-function coefficients $(a, b, c)$, the same exchange market price process $\phi$, and for each trader $i$, the same value function $V^{i}$ and excess-inventory process $z^{i}$.

In equilibrium, whether with linear-quadratic size discovery or with dark-pool size discovery (whether based on linear or proportional rationing), traders are strategic about their influence on the expected terms of trade in subsequent size-discovery sessions through the impacts of their exchange trading on the exchange market price. For example, a trader with a negative excess inventory reduces exchange demands by even more than in the equilibrium without size discovery. Focusing on the particular equilibrium defined by (14)-(16), as $\lambda$ increases, the expected total volume of trade in the double-auction market declines, given the incentive to wait and achieve lower expected execution costs in the next size-discovery session, and also given the incentive to shade bids in order to improve the expected terms of trade in size discovery. All traders are made worse off. If size-discovery sessions are run "too frequently," in that $\lambda>\bar{\lambda}$, these strategic incentives to shade bids become so powerful that the exchange market breaks down (that is, no affine market-clearing optimal demand functions exist).

These same strategic incentives give rise to multiple equilibria in the presence of size discovery. Each trader is more willing to submit large orders if other traders are submitting large orders, since the resulting market depth reduces price impact. Size discovery heightens this strategic complementarity because there are now direct exchange price impacts and spillover effects into the terms of trade for size discovery. The combined strategic complementarity induces multiple equilibria: one in which traders coordinate to produce low market depth and reduced exchange trading and another with higher market depth and more aggressive exchange trading.

Proposition 2 states that the two size-discovery mechanisms that we consider generate the same equilibria. Intuitively, both mechanisms lead to the same efficient allocations by definition. Traders that already have the efficient allocation $z^{i}=\bar{Z}$ when a size-discovery session occurs must receive a cash transfer of zero in equilibrium, and by a straightforward calculation this observation implies that both mechanisms lead to the same equilibrium cash transfers. ${ }^{24}$ It is then intuitive that equilibrium exchange-trading strategies do not depend on which mechanism is used. We formalize this intuition in Appendix C.

We emphasize that traders optimally participate in size discovery and report truthfully in any equilibrium. If we had relaxed our definition of equilibrium so as to include mutual best

\footnotetext{
${ }^{24}$ By equation (6), if traders report truthfully, then the transfer to trader $i$ given an exchange price $p$ is the sum of $p z^{i}$ and a linear-quadratic function $f$ whose $\operatorname{arguments}$ are $Z$ and $p$. The transfer to trader $i$ is zero when $z^{i}=\bar{Z}$, in which case $f(Z)=-\bar{Z} p$.
} 
responses involving no participation by any trader in size discovery, then there would have been one additional equilibrium, with symmetric affine exchange trading and no size-discovery participation, for any $\lambda>0$. This equilibrium is of course equivalent to the symmetric affine equilibrium with no size-discovery venue $(\lambda=0)$.

Likewise, we do not consider equilibria with nonlinear exchange trading strategies. Such equilibria might exist even when $\lambda>\bar{\lambda}$. We only prove that for $\lambda>\bar{\lambda}$, there are no constants $(a, b, c)$ that characterize an equilibrium in affine trading strategies. With affine exchange trading strategies, zero exchange trading volume is inconsistent with the existence of a marketclearing price. Thus, there cannot be an equilibrium with zero exchange trading volume, given our definitions.

\subsection{Intuition}

In this section, we provide intuition for the key message of Propositions 1 and 2, that size discovery reduces welfare. Here, we use only informal arguments. The formal result, stated in Proposition 1, is proved in Appendix B.

In equilibrium, each size-discovery session efficiently reallocates the asset among the traders. However, in equilibrium, size discovery entails two welfare costs. First, traders are less willing to incur price-impact costs on the exchange because they will later have the chance to reduce inventory imbalances with no direct price-impact costs at the next size-discovery session. Traders therefore submit smaller orders than they would in the absence of size discovery, leading to slower reallocation of the asset on the exchange. This harms welfare through increased excess-inventory costs. Second, traders further reduce the magnitudes of their exchange trades in order to mitigate the impact of the exchange trades on the upcoming size-discovery terms of trade, which depend on prior exchange prices. This further reduces welfare by increasing excess-inventory holding costs. In this section, to explain the intuition for our result as simply as possible, we ignore the second of these two welfare-cost channels and merely show that the first cost is exactly offset by the efficiency gain associated with aggressive trade in size-discovery sessions. It follows that, when combined, the two sources of welfare cost associated with size discovery must outweigh the welfare gains.

In order to focus on the first welfare cost, we simplify our setting so as to cut the link between the terms of trade in size discovery and the exchange price-setting mechanism. To this end, for any demand-function coefficients $(a, b, c)$, we let

$$
\phi_{(a, b, c)}\left(Z_{t}\right) \equiv \frac{a+c \bar{Z}_{t}}{-b}
$$


denote the equilibrium exchange price, as a function of the mean excess inventory, $\bar{Z}_{t}$. Rather than the original control problem (8), trader $i$ now chooses exchange demands $D$ and sizediscovery reports $\mu$ to maximize the conditional expectation of

$$
\begin{aligned}
J^{i}\left(D, \mu, t_{0}\right)=z_{\mathcal{T}}^{D, \mu} \pi & -\int_{t_{0}}^{\mathcal{T}}\left[\gamma\left(z_{t}^{D, \mu}\right)^{2}+\Phi_{(a, b, c)}\left(D_{t} ; Z_{t}-z_{t}^{D, \mu}\right) D_{t}\right] d t \\
& +\sum_{\left\{k: t_{0}<\tau_{k}<\mathcal{T}\right\}} T^{i}\left(\left(\mu_{k}, \mu_{k}^{-i}\right), \phi_{(a, b, c)}\left(Z_{\tau_{k}}\right)\right) .
\end{aligned}
$$

In this simplified setting, traders know that if they deviate from equilibrium exchange orders and thus move the market-clearing price, their size-discovery transfers will be unaffected, because size discovery will be based on the equilibrium price (17) rather than on the resulting offequilibrium market-clearing price. Although artificial, this simplifies our analysis by removing the second welfare cost of size discovery mentioned above. That is, in this artificial setting, traders are no longer concerned that their exchange trades will affect future size-discovery transfers.

We now show informally that the first of the welfare costs of size discovery is precisely offset by its benefits. Specifically, we show that if there exists an equilibrium with demand coefficients $(a, b, c)$ for some fixed size-discovery frequency $\lambda$, then there exists an equilibrium with the same value functions for any frequency $\lambda^{\prime} \leq \bar{\lambda}$. To see this, fix some $\lambda$ and demandfunction coefficients $(a, b, c)$ that comprise an equilibrium. Truthful reporting is optimal in equilibrium, so under either of the size-discovery mechanisms considered in the previous section, the equilibrium size-discovery cash transfers are

$$
\phi_{(a, b, c)}\left(Z_{\tau_{k}}\right)\left(z_{\tau_{k}}^{i}-\bar{Z}_{\tau_{k}}\right)
$$

Size-discovery allocations are efficient. The Hamilton-Jacobi-Bellman (HJB) equation for optimal demand submission by trader $i$ is therefore

$$
\begin{aligned}
0 & =-\gamma\left(z^{i}\right)^{2}+r\left(v z^{i}-V\left(z^{i}, Z\right)\right)+\frac{\sigma_{i}^{2}}{2} V_{z z}\left(z^{i}, Z\right)+\frac{\sigma_{Z}^{2}}{2} V_{Z Z}\left(z^{i}, Z\right)+\rho^{i} V_{z Z}\left(z^{i}, Z\right) \\
& +\sup _{D}\left\{-\Phi_{(a, b, c)}\left(D ; Z-z^{i}\right) D+V_{z}\left(z^{i}, Z\right) D\right\}+\lambda\left(V(\bar{Z}, Z)-V\left(z^{i}, Z\right)+\phi_{(a, b, c)}(Z)\left(z^{i}-\bar{Z}\right)\right) .
\end{aligned}
$$

The distinction with our main model setting is that, here, artificially, the exchange demand $D$ has no effect on size-discovery terms of trade. The first-order condition in the HJB maximization problem is

$$
V_{z}\left(z^{i}, Z\right)=\Phi_{(a, b, c)}^{\prime}\left(D ; Z-z^{i}\right) D+\Phi_{(a, b, c)}\left(D ; Z-z^{i}\right) .
$$

Because $(a, b, c)$ constitutes an equilibrium, this first-order condition is solved by the solution 
$D^{i}$ to the fixed-point problem

$$
D^{i}=a+b \Phi_{(a, b, c)}\left(D^{i} ; Z-z^{i}\right)+c z^{i}
$$

A straightforward calculation shows that

$$
D^{i}=c\left(z^{i}-\bar{Z}\right)
$$

so the exchange clearing price is indeed

$$
\Phi_{(a, b, c)}\left(D^{i} ; Z-z^{i}\right)=\phi_{(a, b, c)}(Z) .
$$

Combining $(18),(19)$, and the definition of $\Phi_{(a, b, c)}^{\prime}\left(D ; Z-z^{i}\right)$, we have

$$
V_{z}\left(z^{i}, Z\right)=\phi_{(a, b, c)}(Z)-\frac{c}{b(n-1)}\left(z^{i}-\bar{Z}\right) .
$$

Integration with respect to $z^{i}$ leaves the indirect utility

$$
V\left(z^{i}, Z\right)=\phi_{(a, b, c)}(Z) z^{i}-\frac{c}{b(n-1)} \frac{\left(z^{i}\right)^{2}}{2}+\frac{c}{b(n-1)} z^{i} \bar{Z}+f(Z),
$$

for some $f(Z)$ that does not depend on $z^{i}$. Then,

$$
V(\bar{Z}, Z)-V\left(z^{i}, Z\right)+\phi_{(a, b, c)}(Z)\left(z^{i}-\bar{Z}\right)=\frac{c}{2 b(n-1)}\left(z^{i}-\bar{Z}\right)^{2}
$$

It follows from algebra that

$$
\sup _{D}\left\{-\Phi_{(a, b, c)}\left(D ; Z-z^{i}\right) D+V_{z}\left(z^{i}, Z\right) D\right\}=\frac{-c^{2}}{b(n-1)}\left(z^{i}-\bar{Z}\right)^{2} .
$$

So, for any equilibrium demand coefficients $(a, b, c)$, the HJB equation reduces to

$$
\begin{aligned}
0=- & \gamma\left(z^{i}\right)^{2}+r\left(v z^{i}-V\left(z^{i}, Z\right)\right)+\frac{\sigma_{i}^{2}}{2} V_{z z}\left(z^{i}, Z\right)+\frac{\sigma_{Z}^{2}}{2} V_{Z Z}\left(z^{i}, Z\right)+\rho^{i} V_{z Z}\left(z^{i}, Z\right) \\
& -\frac{c^{2}}{b(n-1)}\left(z^{i}-\bar{Z}\right)^{2}+\lambda \frac{c}{2 b(n-1)}\left(z^{i}-\bar{Z}\right)^{2} .
\end{aligned}
$$


Now, for any alternative size-discovery frequency $\lambda^{\prime}$, we let

$$
\begin{aligned}
c^{\prime} & =c+\frac{\lambda^{\prime}-\lambda}{2} \\
b^{\prime} & =c^{\prime} \frac{b}{c} \\
a^{\prime} & =c^{\prime} \frac{a}{c} .
\end{aligned}
$$

From simple algebra,

$$
\lambda^{\prime} \frac{c^{\prime}}{2 b^{\prime}(n-1)}-\frac{\left(c^{\prime}\right)^{2}}{b^{\prime}(n-1)}=\lambda \frac{c}{2 b(n-1)}-\frac{c^{2}}{b(n-1)} .
$$

It follows that for any $\lambda^{\prime} \in[0, \bar{\lambda}]$, the same value functions $V^{i}$ solve the reduced HJB equation (21) after substituting the new size-discovery frequency $\lambda^{\prime}$ and the new demand coefficients $\left(a^{\prime}, b^{\prime}, c^{\prime}\right)$. In this simplified setting, the optimality of truthful reporting depends on the value functions and the price function $\phi_{(a, b, c)}$, but it is easy to calculate that $\phi_{(a, b, c)}=\phi_{\left(a^{\prime}, b^{\prime}, c^{\prime}\right)}$, so truthful reporting remains optimal. Since $c^{\prime} / b^{\prime}=c / b$ by definition, the exchange demands defined by $\left(a^{\prime}, b^{\prime}, c^{\prime}\right)$ satisfy the first-order condition (18).

This informal argument implies that the equilibrium value functions in this simplified setting do not depend on the mean size-discovery frequency $\lambda .^{25}$ That is, changing $\lambda$ has no effect on welfare. This analysis ignores the second equilibrium channel by which size discovery harms welfare, the reduction of exchange order aggressiveness in order to improve the expected terms of trade in the upcoming size-discovery session. Any increase in $\lambda$ exacerbates this second welfare cost, simply because it increases the likelihood that the next size-discovery session will be based on the current exchange price. The net effect of an increase in $\lambda$ is therefore a reduction in welfare, which is shown rigorously in Appendix B.

\section{$4 \quad$ Further properties}

This section further interprets our model.

\footnotetext{
${ }^{25}$ We provide a formal proof of this result in Appendix D.
} 


\subsection{Welfare}

Because traders maximize their expected total payoffs, welfare in this setting can be captured as the sum of the traders' continuation values, which is

$$
\hat{W}\left(z_{t}\right) \equiv \sum_{i=1}^{n} V^{i}\left(z_{t}^{i}, Z_{t}\right)=\sum_{i=1}^{n} \theta_{i}(\lambda, b)+v Z_{t}-\frac{n \gamma}{r} \bar{Z}_{t}^{2}-K(\lambda, b) \sum_{i=1}^{n}\left(z_{t}^{i}-\bar{Z}_{t}\right)^{2}
$$

In the more efficient equilibrium defined by (14)-(16), welfare is strictly monotonically declining in the mean frequency $\lambda$ of size discovery. That is, for the more efficient equilibrium, welfare gets strictly lower as the frequency of size-discovery sessions is increased, until size-discovery sessions are so frequent that the exchange price-discovery market breaks down. In particular, welfare is strictly lower with size discovery than without size-discovery, the case of $\lambda=0$. Indeed, as stated by Proposition 1, for each $\lambda>0$, in the better equilibrium, each trader's value is strictly declining in the frequency $\lambda$ of size discovery. Although it is individually rational for traders to participate in size-discovery if it is available, all traders would strictly prefer to commit to a market design in which size discovery is not available.

Indeed, reducing size-discovery is better for every trader, regardless of the initial crosssectional distribution $z_{0}$ of the asset. That is, for any mean size-discovery frequencies $\lambda \leq \bar{\lambda}$ and $\lambda^{\prime}<\lambda$, the equilibrium (defined by (14)-(16)) associated with $\lambda^{\prime}$ Pareto dominates that associated with $\lambda$.

\subsection{Ex-post optimality}

Extending from the results of Du and Zhu (2017), our equilibrium strategies are ex-post optimal. That is, for each trader $i$, the equilibrium strategy $\left(D^{i}, \mu^{i}\right)$ also solves the complete-information version of problem (8), in which the information filtration of trader $i$ is artificially replaced with the complete-information filtration $\mathbb{F}$, thus revealing the inventories of all of the other agents. This property follows from the fact that even if equilibrium is redefined by relaxing the measurability restrictions on agent strategies to $\mathbb{F}$-measurability, the equilibrium optimal strategies are unaffected.

\subsection{Perfect Bayes}

Although we are working here for expositional simplicity in a continuous-time setting, each equilibrium that we propose may safely be considered to be a Perfect Bayesian Equilibrium. That is, in light of the ex-post optimality property, beliefs about other traders' inventories are irrelevant. This is tied down rigorously in a discrete-time analogue of our model found 
in Appendix F. In discrete time, the ex-post optimality property implies subgame perfection for the complete-information game. Moreover, the primitive parameters of the discrete-time model and the associated discrete-time equilibrium bidding behavior converge to those for the continuous-time model as the length of a time interval shrinks to zero. This convergence was shown by Duffie and Zhu (2017) for a simpler version of this model, and applies also in the current setting.

\subsection{Equivalent behavior for alternative preference models}

With respect to equilibrium behavior, our model is equivalent ${ }^{26}$ to one in which there is no shock $H_{t}^{i}$ to the level of excess inventory, but there is instead a Lévy process $\eta^{i}$ determining the net rate of benefit at time $t$ to trader $i$ for asset position $z_{t}^{i}$ of $\eta_{t}^{i} z_{t}^{i}-\gamma\left(z_{t}^{i}\right)^{2}$.

Our model is also behaviorally equivalent to an infinite-horizon model in which traders discount payoffs at the time preference rate $r$ and the asset pays dividends continuously at the exogenous rate $r v$, rather than a final lump-sum dividend with mean $v$. This equivalence follows from an inspection of the HJB equation used in Appendix B to prove the optimality ${ }^{27}$ of traders' candidate equilibrium trading and reporting strategies.

\section{Alternative market designs and further implications}

This section considers alternative market designs, and some implications of our findings.

\subsection{The benefit of an initializing size-discovery session}

If the initial aggregate excess inventory $Z_{0}$ is observable, an obvious strict improvement in welfare is obtained by an initializing size-discovery session. For example, Duffie and Zhu (2017)

\footnotetext{
${ }^{26}$ To see this equivalence, suppose that $\eta$ is an exogenous Lévy process, and consider a model with no exogenous excess-inventory shocks in which a trader with position process $y$, determined only by the trader's initial position and trades, benefits at time $t$ at the rate $\eta_{t} y_{t}-\gamma y_{t}^{2}$. This preference model induces the same behavior as that associated with the benefit rate

$$
\eta_{t} y_{t}-\gamma y_{t}^{2}-\frac{\eta_{t}^{2}}{4 \gamma}=-\gamma\left(y_{t}-\frac{\eta_{t}}{2 \gamma}\right)^{2}=-\gamma\left(y_{t}+H_{t}^{i}\right)^{2}
$$

where $H_{t}^{i}=-\eta_{t} /(2 \gamma)$, because the extra term $\eta_{t}^{2} /(4 \gamma)$ merely translates the total value by the constant $E\left(\int_{0}^{\mathcal{T}} \eta_{t}^{2} d t\right) /(4 \gamma)$. This preference model induces the same behavior as that for our basic model in which there is a cost $\gamma\left(z_{t}^{i}\right)^{2}$ for a position process $z_{t}^{i}=y_{t}+H_{t}^{i}$ that is determined by trade and by an exogenous Lévy excess-inventory shock process $H_{t}^{i}$. By similar arguments, our model is also behaviorally equivalent to a model that includes both an excess-inventory shock process and a preference shock process.

${ }^{27}$ Verification of optimality follows from the HJB equation and "transversality" arguments similar to those in Appendix B.
} 
showed an improvement in welfare through a workup session at time zero, before the market opens. Workup, however, does not efficiently reallocate the initial inventories. Appendix A shows that a size-discovery session at time zero can in principle achieve a perfect initial allocation, if the mechanism designer is given information about the initial aggregate excess inventory $Z_{0}$. We have shown, however, that welfare is not improved by running size discovery after time zero, even though the traders' inventories are perfectly reallocated at each size-discovery session. The benefits of size discovery are offset by the dampening of order flow on the exchange market caused by the prospect of future size-discovery sessions.

\subsection{Does reducing the efficiency of size discovery help?}

One might be drawn to conjecture that the mechanism designs for size discovery that we have analyzed are simply "too efficient." Indeed, the allocative efficiency and low effective price impacts of our size-discovery mechanism designs offer such an attractive alternative for executing trades, relative to submitting orders into the price-discovery market, that all of the benefits of adding size discovery are more than offset by lost gains from trade in the exchange market.

Given this tension, one might hope to impair the efficiency of the size-discovery design just enough to raise overall expected gains from trade. By this line of enquiry, one would look for a loss of size-discovery efficiency that is more than offset by a gain in price-discovery allocative efficiency through an improvement of market depth.

We have discovered that this approach does not work, at least among linear-quadratic schemes for size discovery. In Appendix $G$, we calculate a mechanism design in which the imbalance $z_{t-}^{i}-\bar{Z}_{t}$ in the excess inventory of trader $i$ is not completely eliminated in the size-discovery session. Instead, only a specified fraction $\xi$ of this imbalance is erased by size discovery. For this analysis, we take the simpler case in which the aggregate excess-inventory process $Z$ is observable to the size-discovery platform operator. ${ }^{28}$ In this setting, any sizediscovery efficiency parameter $\xi$ between 0 and 1 can be supported in equilibrium. As shown in Appendix G, all traders' value functions, and thus overall welfare, are invariant to the size-discovery efficiency parameter $\xi$. That is, welfare is the same whether one runs perfect reallocation mechanisms $(\xi=1)$, arbitrarily imperfect size-discovery mechanisms $(0<\xi<1)$, or no size-discovery mechanisms at all. For the case in which $Z_{t}$ is unobservable, our unreported numerical analysis shows that welfare is strictly lower with impaired size-discovery mechanisms than with no mechanisms at all.

\footnotetext{
${ }^{28}$ We also slightly modify our notion of budget balance. Given the equilibrium strategies, the mechanism is budget balanced with probability 1 , but this might not be the case for arbitrary off-equilibrium reports.
} 


\subsection{Eliminating the exchange market}

It is natural to ask whether simply getting rid of the price-discovery exchange market and running only size-discovery sessions could improve welfare, relative to a setting with only price discovery. Even if such a radical redesign of markets could be realistically contemplated, in all of the cases that we have studied, the size-discovery scheme must either violate individual rationality or rely unrealistically on information about the aggregate market supply $Z_{t}$.

In Appendix H, we take the setting of Section 3 except that (i) there is no exchange (pricediscovery) market and (ii), given the lack of price information for setting the terms of trade in size discovery, the aggregate excess inventory $Z_{t}$ is assumed to be observable to the sizediscovery platform operator. We show that there is a unique equilibrium for the associated dynamic game, and that the first-best allocation is achieved in the limit as the frequency of size-discovery sessions approaches infinity.

For the more realistic case of unobservable $Z_{t}$, we show in Appendix I that an altered version of our linear-quadratic size-discovery mechanism, run continuously (non-stop), can achieve the first-best allocation in equilibrium. However, in the more realistic case in which the platform operator cannot directly observe the aggregate supply $Z_{t}$, it is impossible to make participation in this mechanism individually rational. Other mechanisms might be able to do better. For example, in unreported results, we have found that the dynamic pivot mechanism of Bergemann and Välimäki (2010) achieves the efficient allocation in a discrete-time version of our primitive model setting. However, the notion of individual rationality associated with that mechanism is highly restrictive in practical market settings. Here, in order for participation to be individually rational, a trader who fails to participate in any mechanism session must be permanently banned from future mechanism sessions.

In summary, even if it were practically feasible to eliminate exchange markets, it seems difficult to replace exchange trading with efficient forms of size discovery without some form of forced participation.

\subsection{Size discovery can arise as a coordination failure}

Our results imply that there may be a tenuous relationship between the operators of sizediscovery and price-discovery platforms, respectively. Barring "omniscient" alternative information sources, size-discovery platform operators rely heavily on lit-exchange price discovery to set the terms of trade in size discovery. However, a size-discovery venue operator can draw volume away from price-discovery markets by holding frequent size-discovery sessions. The CFA Institute (2012) addresses general concerns in this area, summarizing with a comment that "The results of our analysis show that increases in dark pool activity and internalization 
are associated with improvements in market quality, but these improvements persist only up to a certain threshold. When a majority of trading occurs in undisplayed venues, the benefits of competition are eroded and market quality will likely deteriorate."

The conflicting incentives of independent lit-exchange and size-discovery venue operators could in some cases lead toward integration of the sponsors of price-discovery platforms and size-discovery platforms for trading the same asset, along the lines of BrokerTec, which operates both of these protocols for treasuries trading on the same screen-based platform. ${ }^{29}$ If, however, an operator of price-discovery and size-discovery platforms were to place tight volume restrictions on its size-discovery platform in order to maintain the depth of its price-discovery platform, a competing platform operator could enter and attract volume into its own size-discovery platform. For example, suppose an integrated operator were to allow traders to participate in size-discovery sessions only to the extent that they contribute to exchange market depth. A competing platform operator could then open an alternative size-discovery venue with no such restriction. The entering size-discovery platform operator could earn rents, for example in the form of fees or profits on cross-services, because it is often strictly beneficial for traders to participate in size-discovery sessions. (In our model, any trader $i$ whose current excess inventory $z_{t}^{i}$ is not equal to the average excess inventory $\bar{Z}_{t}$ has a strictly positive private benefit associated with participation in a size-discovery session held at time $t$.) The entering service provider does not internalize the costs to an incumbent exchange operator of lost volume-related fees, nor to market participants for reduced allocative efficiency. That is, competition among trade venue operators can lead to a coordination failure.

As we noted in the introduction, regulators have attempted to cure market-coordination failures that they associate with size discovery. In 2018, for example, the European Union placed strict caps on volumes of trade executed in dark pools. Johann, Putnins, Sagade, and Westheide (2019), however, found that these rules have been evaded with "quasi-dark" trading mechanisms. These include internal crossing, by which a broker-dealer matches its own customers' buy and sell orders internally at the exchange price, rather than sending these orders to the exchange. As another implicit form of size-discovery trade that is permitted by Section 17a-7 of the Investment Company Act of 1940, a mutual fund management firm is permitted to trade assets between the different funds that it manages, at the "independent current market price."

Zhu (2014) has shown that in a setting with asymmetric information about asset payoffs, there tends to be a selection bias by which relatively informed investors migrate toward price-

\footnotetext{
${ }^{29}$ Even in this case, however, Schaumburg and Yang (2016) point to some interference arising from price information arriving during size-discovery sessions from the simultaneous operation of Treasury futures trading on the Chicago Mercantile Exchange.
} 
discovery markets and relatively less informed investors migrate toward dark pools. This seems to suggest support for robust trade volumes on both types of venues. On the other hand, Zhu (2014) addresses the case of dark pools that promote this selection effect with delays in dark-pool order execution caused by rationing, because rationing discourages informed investors who want to act quickly on their information. As we have pointed out, dark-pool rationing is a relatively crude mechanism design for size-discovery. Although we have not analyzed the implications in our setting of adding asymmetric information about asset payoffs, one may anticipate from our results that more efficient mechanism designs than those currently used in dark pools would be less discouraging to informed investors. This could call into question the robustness of a market design that allows size-discovery venues to free-ride on the price information coming from lit exchanges, while also having a significant ability to draw volume away from lit exchanges.

\section{References}

Almgren, R., and N. Chriss. 2001. Optimal execution of portfolio transactions. Journal of Risk $3: 5-40$.

Arrow, K. 1979. The property rights doctrine and demand revelation under incomplete information. In M. Boskin, ed., Economics and Human Welfare. Academic Press, Cambridge, Massachusetts.

Association Française des Marchés Financiers. 2019. Impact of the mifir volume cap mechanism on the microstructure of european equity markets. AMAFI, Paris. https://www . google.com/url?sa=t\&rct=j\&q=\&esrc=s\&source=web\&cd=\&ved= 2ahUKEwjqpten8_fpAhVKFzQIHdtGCpAQFjAAegQIAxAB\&url=http $\% 3 A \% 2 F \% 2 F w w w$. amafi . fr\%2Fdownload\%2Fpages\%2Fd1UWBwfYFt17f4sypgw1Nu11BP82vhbbgaPWbHOV . pdf\&usg= AOvVaw1fgDR6rsH-s9rokQJe31Vd.

Athey, S., and I. Segal. 2013. An efficient dynamic mechanism. Econometrica 81:2463-85.

Bergemann, D., and J. Välimäki. 2010. The dynamic pivot mechanism. Econometrica 78:77189.

BGC. 2015. BGC Derivative Markets, L.P. Rules. https://www.cftc.gov/sites/default/ files/groups/public/@otherif/documents/ifdocs/orgsefbgcexhibitm160128.pdf.

Budish, E., P. Cramton, and J. Shim. 2015. The high-frequency trading arms race: Frequent batch auctions as a market design response. Quarterly Journal of Economics 130:1547-621. 
Buti, S., B. Rindi, and I. M. Werner. 2011. Diving into dark pools. Working Paper, Fisher College of Business, Ohio State University. https://papers.ssrn.com/sol3/papers.cfm? abstract_id=1630499.

CFA Institute. 2012. Dark pools, internalization, and equity market quality. White paper, CFA Institute. https://www.cfapubs.org/doi/pdf/10.2469/ccb.v2012.n5.1.

Clarke, E. 1971. Multipart pricing of public goods. Public Choice 2:19-33.

Collin-Dufresne, P., B. Junge, and A. B. Trolle. 2020. Market structure and transaction costs of index CDSs. Journal of Finance forthcoming https://doi.org/10.1111/jofi.12953.

d'Aspremont, C., and L.-A. Gérard-Varet. 1979. Incentives and incomplete information. Journal of Public Economics 11:25-45.

Degryse, H., F. De Jong, and V. van Kervel. 2015. The impact of dark trading and visible fragmentation on market quality. Review of Finance 19:1587-622.

Division of Trading and Markets. 2013. Equity market structure literature review, Part I: market fragmentation. U.S. Securities and Exchange Commission, https://www.sec.gov/ marketstructure/research/fragmentation-lit-review-100713.pdf.

Du, S., and H. Zhu. 2017. What is the optimal trading frequency in financial markets? Review of Economic Studies 84:1606-51.

Duffie, D., and H. Zhu. 2017. Size discovery. The Review of Financial Studies 30:1095-150.

Dworczak, P. 2020. Mechanism design with aftermarkets: Cutoff mechanisms. Econmetrica forthcoming.

European Securities and Markets Authority. 2020. Consultation paper mifid ii/ mifir review report on the transparency regime for equity and equity-like instruments, the double volume cap mechanism and the trading obligations for shares. ESMA, Paris.

Farley, R., E. Kelley, and A. Puckett. 2017. Dark trading volume and market quality: A natural experiment. Working paper, University of Tennessee, Knoxville. http://www1.villanova. edu/content/dam/villanova/VSB/assets/marc/marc2018/SSRN-id3088715.pdf.

Fleming, M., and G. Nguyen. 2015. Order flow segmentation and the role of dark trading in the price discovery of U.S. treasury securities. Working Paper, Federal Reserve Bank of New York. https://www. newyorkfed.org/medialibrary/media/research/ staff_reports/sr624.pdf. 
Fleming, M., E. Schaumburg, and R. Yang. 2015. The evolution of workups in the U.S. treasury securities market. Liberty Street Economics, Federal Reserve Bank of New York. http://libertystreeteconomics.newyorkfed.org/2015/08/ the-evolution-of-workups-in-the-us-treasury-securities-market.html.

Fudenberg, D., and J. Tirole. 1991. Game theory. Cambridge, Massachusetts: MIT press.

German Ministry of Finance. 2019. Necessary amendments and revisions to secondary market provisions in MiFID and MiFIR. Position paper, German Ministry of Finance. https://www. bundesfinanzministerium.de/Content/DE/Standardartikel/ Themen/Internationales_Finanzmarkt/Positionspapiere-Mifid-Mifir.html.

GFI. 2015. GFI Swaps Exchange LLC rulebook. GFI Technical Report. https://www.cftc.gov/sites/default/files/stellent/groups/public/@otherif/ documents/ifdocs/exhibitm1rulebookgfiswaps.pdf.

Giancarlo, J. C. 2015. Pro-reform reconsideration of the CFTC swaps trading rules: Return to Dodd-Frank. Commodity Futures Trading Commission Technical Report. https://www.cftc.gov/sites/default/files/idc/groups/public/@newsroom/ documents/file/sefwhitepaper012915.pdf.

Groves, T. 1973. Incentives in teams. Econometrica 41:617-31.

Hatheway, F., A. Kwan, and H. Zheng. 2017. An empirical analysis of market segmentation on U.S. equity markets. Journal of Financial and Quantitative Analysis 52:2399-427.

Johann, T., T. Putnins, S. Sagade, and C. Westheide. 2019. Quasi-dark trading: The effects of banning dark pools in a world of many alternatives. U.S. Securities and Exchange Commission, http://www.fmaconferences .org/Glasgow/Papers/working_paper.pdf.

Klemperer, P. D., and M. A. Meyer. 1989. Supply function equilibria in oligopoly under uncertainty. Econometrica 57:1243-77.

Liu, S., J. Wang, and C. Wu. 2015. Liquidity frictions, trading and volatility: Evidence from the us treasury market. Washington State University, Working paper. http://www . fmaconferences.org/Sydney/Papers/TradingandVolatility_LiuWangWu_FMAAsia.pdf.

Myerson, R. B., and M. A. Satterthwaite. 1983. Efficient mechanisms for bilateral trading. Journal of economic theory 29:265-81.

Nimalendran, M., and S. Ray. 2014. Informational linkages between dark and lit trading venues. Journal of Financial Markets 17:230-61. 
Ollár, M., M. Rostek, and J. H. Yoon. 2017. Privacy in markets. Working paper, Department of Economics, University of Wisconsin, Madison. https://papers.ssrn.com/sol3/papers. cfm?abstract_id=3071374.

Pancs, R. 2014. Workup. Review of Economic Design 18:37-71.

Pavan, A., I. Segal, and J. Toikka. 2014. Dynamic mechanism design: A Myersonian approach. Econometrica 82:601-53.

Protter, P. 2005. Stochastic integration and differential equations, second edition. Heidelberg: Springer.

Rostek, M., and M. Weretka. 2012. Price inference in small markets. Econometrica 80:687-711.

—. 2015. Dynamic thin markets. Review of Financial Studies 28:2946-92.

Sannikov, Y., and A. Skrzypacz. 2016. Dynamic trading: Price inertia and frontrunning. Working paper, Graduate School of Business, Stanford University. https://economics.uchicago.edu/sites/economics.uchicago.edu/files/uploads/ $\mathrm{PDF} /$ sannikov_dynamic_trading.pdf.

Schaumburg, E., and R. Yang. 2016. The workup, technology, and price discovery in the interdealer market for U.S. treasury securities. Liberty Street Economics, Federal Reserve Bank of New York. http://libertystreeteconomics.newyorkfed.org/2016/02/ the-workup-technology-and-price-discovery-in-the-interdealer-market-for-us-treasury-se html.

SIFMA. 2016. SIFMA electronic bond trading report: US corporate \& municipal securities. Technical Report, Securities Industry and Financial Markets Association. https://www.sifma.org/wp-content/uploads/2017/05/ sifma-electronic-bond-trading-report-us-corporate-and-municipal-securities. pdf.

Tradeweb. 2014. Market regulation advisory notice - work-up protocol. Tradeweb Technical Report. http://www.tradeweb.com/uploadedFiles/Tradeweb/Content/About_Us/ Regulation/DW\%20SEF\%20MRAN\%20-\%20Work-Up\%20Protocol\%20(12.29.14)v2.pdf.

Tradition. 2015. Tradition SEF platform supplement. Tradition Technical Report. http:// WWw.traditionsef . com/assets/regulatory/rulebooks/Rulebook-2015-05.pdf.

Vayanos, D. 1999. Strategic trading and welfare in a dynamic market. The Review of Economic Studies 66:219-54. 
Vickrey, W. 1961. Counterspeculation, auctions and competitive sealed tenders. Journal of Finance 16:8-37.

Vives, X. 2011. Strategic supply function competition with private information. Econometrica 79:1919-66.

Walras, L. 1877. Eléments d'economie politique pure, fourth edition. Lausanne: L. Corbas.

Wholesale Markets Brokers' Association. 2012. Comment for proposed rule 77 FR 38229. WMBA Technical Report. https://comments.cftc.gov/publiccomments/ViewComment. aspx?id=58343.

Wilson, R. 1979. Auctions of shares. Quarterly Journal of Economics 93:675-89.

Ye, L. 2016. Understanding the impacts of dark pools on price discovery. Working paper, The Chinese University of Hong Kong, Shenzhen. https://arxiv.org/pdf/1612.08486.pdf.

Zhu, H. 2014. Do dark pools harm price discovery? Review of Financial Studies 27:747-89. 


\section{Appendices}

The appendices provide auxiliary results and proofs.

\section{A A mechanism design for size discovery}

Our dynamic trading game involves continual exchange trading punctuated by occasional sizediscovery sessions. This appendix focuses on a static setting and on a linear-quadratic (LQ) class of mechanism designs for size-discovery sessions. This mechanism design is tractable when inserted into the dynamic setting of our main model. We show that this LQ class of mechanism designs contains a mechanism that is efficient, budget balanced, strategy proof, and ex-post individually rational.

Our mechanism designer, say a trade platform operator, elicits reports from each of the $n$ traders about their asset positions, and based on those reports makes cash and asset transfers. For the purposes of this appendix, we will initially assume that the platform operator can observe the current aggregate inventory ${ }^{30}$ or equivalent information. In our main application, this equivalent information is obtained from the immediately prior exchange price. Without loss of generality, we take $t=0$, and denote $Z_{0}$ simply as $Z$.

A report from trader $i$ is a random variable $\hat{z}^{i}$ that is measurable with respect to the information set $\mathcal{F}_{0}^{i}$ of trader $i$. Given a list $\hat{z}=\left(\hat{z}^{1}, \ldots, \hat{z}^{n}\right)$ of trader reports, a reallocation is a list $y=\left(y^{1}, \ldots, y^{n}\right)$ of finite-variance random variables that is measurable with respect to ${ }^{31}$ $\{Z, \hat{z}\}$ and satisfies $\sum_{i=1}^{n} y^{i}=0$.

Anticipating the form of post-mechanism indirect utility for the equilibrium of our eventual model of a dynamic market, we assume that the value to trader $i$ of a given reallocation $y$ is $\mathbb{E}\left[V^{i}\left(z_{0}^{i}+y^{i}, \bar{Z}\right) \mid \mathcal{F}_{0}^{i}\right]$, where $\bar{Z} \equiv Z / n$ and $V^{i}: \mathbb{R}^{2} \rightarrow \mathbb{R}$ is of the form

$$
V^{i}\left(z^{i}, \bar{Z}\right)=u^{i}(\bar{Z})+\left(\beta_{0}+\beta_{1} \bar{Z}\right)\left(z^{i}-\bar{Z}\right)-K\left(z^{i}-\bar{Z}\right)^{2},
$$

where $u^{i}: \mathbb{R} \rightarrow \mathbb{R}$ is a real-valued measurable function to be specified such that $u^{i}(\bar{Z})$ has a finite expectation and $\beta_{0}, \beta_{1}$, and $K$ are real numbers, with $K>0$, that do not depend on $i$.

In our application, the value $V^{i}\left(z^{i}, \bar{Z}\right)$ is measured in units of wealth, allowing us to use a simple additive welfare criterion. A reallocation is thus welfare maximizing given a list $\hat{z}$ of reports if it solves

$$
\sup _{y \in \mathcal{Y}(\hat{z}, Z)} \mathbb{E}\left[\sum_{i=1}^{n} V^{i}\left(z_{0}^{i}+y^{i}, \bar{Z}\right)\right],
$$

where $\mathcal{Y}(\hat{z}, Z)$ is the set of reallocations. A reallocation is said to be perfect if it is welfare maximizing for the case in which the reports are perfectly revealing, ${ }^{32}$ for example when $\hat{z}^{i}=z_{0}^{i}$. From the quadratic costs of asset dispersion across traders reflected in the last term of $V^{i}\left(z^{i}, \bar{Z}\right)$, it is immediate that a reallocation $y$ is perfect if and only if $z_{0}^{i}+y^{i}=\bar{Z}$ for all $i$.

\footnotetext{
${ }^{30}$ We use the terms "inventory" and "excess inventory" interchangeably.

${ }^{31}$ That is, $z$ is measurable with respect to the sub- $\sigma$-algebra of $\mathcal{F}$ generated by $\{\hat{z}, Z\}$.

${ }^{32} \mathrm{~A}$ report $\hat{z}^{i}$ from trader $i$ is perfectly revealing if $z_{0}^{i}$ is measurable with respect to $\left\{Z, \hat{z}^{i}\right\}$.
} 
We will now derive a mechanism that achieves a perfect reallocation. Specifically, a mechanism is a function that maps $Z$ and a list $\hat{z}$ of reports to a reallocation denoted $Y(\hat{z})=$ $\left(Y^{1}(\hat{z}), \ldots, Y^{n}(\hat{z})\right)$ and a list $T(\hat{z}, Z)=\left(T^{1}(\hat{z}, Z), T^{2}(\hat{z}, Z), \ldots, T^{n}(\hat{z}, Z)\right)$ of real-valued "cash" transfers with finite expectations. In the game induced by a mechanism $(Y, T), \hat{z}$ is an equilibrium if, for each trader $i$, the report $\hat{z}^{i}$ solves

$$
\sup _{\mu} U^{i}\left(\left(\mu, \hat{z}^{-i}\right)\right)
$$

where, for any list $\hat{z}$ of reports,

$$
U^{i}(\hat{z})=\mathbb{E}\left[V^{i}\left(z_{0}^{i}+Y^{i}(\hat{z}), \bar{Z}\right)+T^{i}(\hat{z}, Z) \mid \mathcal{F}_{0}^{i}\right]
$$

and where we adopt the standard notation by which for any $x \in \mathbb{R}^{n}$ and $w \in \mathbb{R}$,

$$
\left(w, x^{-i}\right) \equiv\left(x^{1}, x^{2}, \ldots, x^{i-1}, w, x^{i+1}, \ldots, x^{n}\right) .
$$

In words, each trader $i$ takes the strategies of the other traders as given and chooses a report $\hat{z}^{i}$ depending only on the information available to trader $i$ that maximizes the conditional expected sum of the reallocated asset valuation and the cash transfer.

For any constant $\kappa_{0}<0$ and any Lipschitz-continuous functions $\kappa_{1}: \mathbb{R} \rightarrow \mathbb{R}$ and $\kappa_{2}:$ $\mathbb{R} \rightarrow \mathbb{R}$ of the commonly observed aggregate inventory $Z$, we will consider the properties of the mechanism $\mathcal{M}^{\kappa}$ defined by the asset reallocation

$$
Y^{i}(\hat{z})=\frac{\sum_{j=1}^{n} \hat{z}^{j}}{n}-\hat{z}^{i}
$$

and the cash transfer

$$
T_{\kappa}^{i}(\hat{z}, Z)=\kappa_{1}(Z) \hat{z}^{i}+\kappa_{0}\left(n \kappa_{2}(Z)+\sum_{j=1}^{n} \hat{z}^{j}\right)^{2}+\kappa_{1}(Z) \kappa_{2}(Z)+\frac{\kappa_{1}^{2}(Z)}{4 \kappa_{0} n^{2}}
$$

The first term of (26) is analogous to compensation at a fixed marginal price of $\kappa_{1}(Z)$. In Section 3.6, where we embed our size-discovery mechanism into a dynamic market game, the "frozen price" $\kappa_{1}(Z)$ is, in equilibrium, almost surely equal to the immediately preceding exchange market price $P(\bar{Z})$.

Departing from forms of size discovery that are used in practice, we include the non-linear second term of (26) in order to force trader $i$ to internalize some of the quadratic cost of an uneven cross-sectional distribution of the asset. The sum of the final two terms in (26) comprise a fixed participation fee, which ensures that the platform operator does not lose money. That is, for any list $\hat{z}$ of reports, the mechanism $\mathcal{M}^{\kappa}$ always leaves a weakly positive profit for the platform operator because $\sum_{i=1}^{n} T_{\kappa}^{i}(\hat{z}, Z) \leq 0$. In Section 3.6, we show that the dark-pool mechanism, which simply posts a fixed price of $\kappa_{1}(Z)$, has equivalent equilibrium allocative properties. Unlike the simpler dark-pool mechanism, however, the linear-quadratic mechanism $\mathcal{M}^{\kappa}$ is strategy proof, as we will now demonstrate.

The following proposition characterizes equilibrium for the mechanism reporting game. The 
proposition also shows that for a carefully chosen $\kappa_{0}$, each trader can actually ignore the reporting strategies of other traders.

\section{A.1 Equilibrium of the mechanism design}

Proposition 3. Consider a mechanism of the form $\mathcal{M}^{\kappa}$, defined by any $\kappa_{0}<0$, and any Lipschitz-continuous $\kappa_{1}(\cdot)$ and $\kappa_{2}(\cdot)$.

1. Suppose trader $i$ anticipates that, for each $j \neq i$, trader $j$ will submit the report $\hat{z}^{j}=$ $z_{0}^{j}$. There is a unique solution to the optimal report problem for trader $i$ induced by the mechanism $\mathcal{M}^{\kappa}$. This solution is $\hat{z}^{i}=z_{0}^{i}$ almost surely, if and only if

$$
\kappa_{2}(Z)=-\bar{Z}+\frac{-\kappa_{1}(Z)+\left(\frac{n-1}{n}\right)\left(\beta_{0}+\beta_{1} \bar{Z}\right)}{2 \kappa_{0} n} .
$$

That is, $\mathcal{M}^{\kappa}$ is a direct revelation mechanism if and only if $\kappa_{2}(Z)$ is given by (27).

2. Suppose $\kappa_{2}(Z)$ is given by (27). If trader $i$ anticipates the report $\hat{z}^{j}=z_{0}^{j}$ for each $j \neq i$, then the truthful report $z^{* i}=z_{0}^{i}$ is ex-post optimal, that is, optimal whether or not we take the special case in which trader $i$ observes ${ }^{33} z_{0}^{-i}$.

3. For the list $z^{*}=\left(z^{* 1}, \ldots, z^{* n}\right)$ of such truthful reports, the reallocation $Y\left(z^{*}\right)$ of (25) is perfect. That is, $z_{0}^{i}+Y^{i}\left(z^{*}\right)=\bar{Z}$ for all $i$.

4. For any $\kappa_{1}(\cdot)$, for $\kappa_{2}(Z)$ given by (27), and for $\kappa_{0}=-K(n-1) / n^{2}$, the mechanism $\mathcal{M}^{\kappa}$ is strategy proof. That is, the truthful report $z^{* i}=z_{0}^{i}$ is a dominant strategy, being an optimal report for trader $i$ regardless of the conjecture by trader $i$ of the reports $\hat{z}^{-i}$ of the other traders.

Proof: Fix a continuation value function $V^{i}$ for trader $i$, given by (23). In equilibrium, trader $i$ achieves the value

$$
\sup _{\hat{z}^{i}} \mathbb{E}\left[V^{i}\left(z_{0}^{i}+Y^{i}(\hat{z}), \bar{Z}\right)+T_{\kappa}^{i}(\hat{z}, Z) \mid \mathcal{F}_{0}^{i}\right]
$$

Fix reports $\hat{z}^{j}=z_{0}^{j}$ for $j \neq i$. Substituting (23) into (28), the quantity inside the expectation of $(28)$ is

$$
\begin{aligned}
& u^{i}(\bar{Z})+\left(\beta_{0}+\beta_{1} \bar{Z}\right)\left(z_{0}^{i}+Y^{i}(\hat{z})-\bar{Z}\right)-K\left(z_{0}^{i}+Y^{i}(\hat{z})-\bar{Z}\right)^{2} \\
& \quad+\kappa_{0}\left(n \kappa_{2}(n \bar{Z})+\sum_{j=1}^{n} \hat{z}^{j}\right)^{2}+\kappa_{1}(n \bar{Z})\left(\hat{z}^{i}+\kappa_{2}(n \bar{Z})\right)+\frac{\kappa_{1}^{2}(n \bar{Z})}{4 \kappa_{0} n^{2}} .
\end{aligned}
$$

We can write

$$
Y^{i}(\hat{z})=\frac{\sum_{j=1}^{n} \hat{z}^{j}}{n}-\hat{z}^{i}=\frac{Z-z_{0}^{i}}{n}-\frac{n-1}{n} \hat{z}^{i} .
$$

The terms in (29) that depend on $\hat{z}^{i}$ sum to

\footnotetext{
${ }^{33}$ To be able to observe $z_{0}^{-i}$ means that $z_{0}^{-i}$ is measurable with respect to $\mathcal{F}_{0}^{i}$.
} 


$$
\left(\beta_{0}+\beta_{1} \bar{Z}\right)\left(-\frac{n-1}{n} \hat{z}^{i}\right)-K\left(\frac{n-1}{n}\right)^{2}\left(z_{0}^{i}-\hat{z}^{i}\right)^{2}+\kappa_{0}\left(n \kappa_{2}(Z)+Z-z_{0}^{i}+\hat{z}^{i}\right)^{2}+\kappa_{1}(Z) \hat{z}^{i} .
$$

The first derivative of this expression with respect to $\hat{z}^{i}$ is

$$
\left(\beta_{0}+\beta_{1} \bar{Z}\right)\left(-\frac{n-1}{n}\right)+2 K\left(\frac{n-1}{n}\right)^{2}\left(z_{0}^{i}-\hat{z}^{i}\right)+2 \kappa_{0}\left(n \kappa_{2}(Z)+Z-z_{0}^{i}+\hat{z}^{i}\right)+\kappa_{1}(Z) .
$$

The second derivative of (29) with respect to $\hat{z}^{i}$ is negative because $K>0$ and $\kappa_{0}<0$. It follows that the unique solution of this first-order condition is the unique optimal report. Substituting $\hat{z}^{i}$ with $\hat{z}^{i}=z_{0}^{i}$ in the first derivative and then equating the result to 0 implies that

$$
0=\left(\beta_{0}+\beta_{1} \bar{Z}\right)\left(-\frac{n-1}{n}\right)+2 \kappa_{0}\left(n \kappa_{2}(Z)+Z\right)+\kappa_{1}(Z)
$$

Thus, for any fixed $\kappa_{1}(\cdot)$ and $\kappa_{0}$, we find that

$$
\kappa_{2}(Z)=-\bar{Z}+\frac{-\kappa_{1}(Z)+\left(\frac{n-1}{n}\right)\left(\beta_{0}+\beta_{1} \bar{Z}\right)}{2 \kappa_{0} n}
$$

is the unique choice for $\kappa_{2}(Z)$ with the property that trader $i$ optimally reports $\hat{z}^{i}=z_{0}^{i}$. Since this report maximizes the quantity inside the expectation of (28), it maximizes the objective function, state by state. This reporting strategy therefore constitutes an ex-post equilibrium of the mechanism game. At the equilibrium reports, we have

$$
\frac{\sum_{j=1}^{n} \hat{z}^{j}}{n}-\hat{z}^{i}=-\left(z_{0}^{i}-\bar{Z}\right)
$$

Thus, $z_{0}^{i}+Y^{i}(\hat{z})=\bar{Z}$, as desired.

For the special case in which

$$
\kappa_{0}=\frac{-K(n-1)}{n^{2}}
$$

we can define $Q \equiv \sum_{j \neq i} \hat{z}^{j} / n$ and calculate that

$$
\begin{aligned}
\kappa_{0}\left(\sum_{j=1}^{n} \hat{z}^{j}\right)^{2}-K\left(z_{0}^{i}+Y^{i}\left(\left(\hat{z}^{i}, \hat{z}^{-i}\right)\right)-\bar{Z}\right)^{2} \\
=\kappa_{0}(n Q)^{2}+\kappa_{0}\left(\hat{z}^{i}\right)^{2}+2 \kappa_{0} n Q \hat{z}^{i}-K\left(z_{0}^{i}+Q-\bar{Z}\right)^{2}-K\left(\frac{n-1}{n}\right)^{2}\left(\hat{z}^{i}\right)^{2} \\
\quad+2 K \frac{n-1}{n} \hat{z}^{i}\left(z_{0}^{i}+Q-\bar{Z}\right) \\
=\kappa_{0}(n Q)^{2}+\kappa_{0}\left(\hat{z}^{i}\right)^{2}-K\left(z_{0}^{i}+Q-\bar{Z}\right)^{2}-K\left(\frac{n-1}{n}\right)^{2}\left(\hat{z}^{i}\right)^{2}+2 K \frac{n-1}{n} \hat{z}^{i}\left(z_{0}^{i}-\bar{Z}\right) .
\end{aligned}
$$


It is thus clear from equation (29) that the optimal report does not depend on $Q$. In this case, $\hat{z}^{i}=z_{0}^{i}$ is therefore a dominant strategy.

By the ex-post optimality property stated in Part 2 of the proposition, it is a Nash equilibrium $^{34}$ of the complete-information game (in which all traders know $z_{0}$ ) for traders to submit the list $z^{*}$ of reports. For the special case $\kappa_{0}=-K(n-1) / n^{2}$, this is the unique Nash equilibrium because, for any trader $i$, the report $z^{* i}$ is a dominant strategy and because of the strict concavity of $U^{i}\left(\left(\mu, \hat{z}^{-i}\right)\right)$ with respect to $\mu$.

\section{A.2 Individual rationality}

We now consider whether trader $i$ could do better by not entering the mechanism at all. From this point, we always fix $\kappa_{2}(\cdot)$ as specified by (27). For arbitrary $\kappa_{0}$ and $\kappa_{1}(\cdot)$, the mechanism $\mathcal{M}^{\kappa}$ need not be individually rational. That is, there could be realizations of $\left(z_{0}^{i}, Z\right)$ at which trader $i$ would strictly prefer $V^{i}\left(z_{0}^{i}, \bar{Z}\right)$ over the expected equilibrium value to trader $i$. However, because the platform operator observes $Z$, he or she can choose $\kappa_{1}(Z)$ so as to ensure that all traders strictly prefer to participate in the mechanism, except in the trivial case in which the initial allocation is already perfect. That our equilibrium is budget balanced, efficient, incentive compatible, and individually rational might at first seem surprising given the results of Myerson and Satterthwaite (1983). Our equilibrium properties are possible because of the reliance of the mechanism on $Z$ for additional information.

Proposition 4. Fix $\kappa_{2}(\cdot)$ as in $(27)$, let $\kappa_{1}(Z)=\beta_{0}+\beta_{1} \bar{Z}$, and let $\kappa_{0}$ be arbitrary. For the equilibrium reports $z^{*}$ of the mechanism $\mathcal{M}^{\kappa}$, we have

$$
U^{i}\left(z^{*}\right)=V^{i}\left(z_{0}^{i}, \bar{Z}\right)+K\left(z_{0}^{i}-\bar{Z}\right)^{2} .
$$

With probability one, trader $i$ weakly prefers this equilibrium value to the value $V\left(z_{0}^{i}, \bar{Z}\right)$ of the initial inventory $z_{0}^{i}$. That is,

$$
U^{i}\left(z^{*}\right)=V^{i}\left(z_{0}^{i}+Y^{i}\left(z^{*}\right), \bar{Z}\right)+T_{\kappa}^{i}\left(z^{*}, Z\right) \geq V^{i}\left(z_{0}^{i}, \bar{Z}\right) .
$$

The inequality is strict unless $z_{0}^{i}=\bar{Z}$. Provided that the probability distribution of $z_{0}$ has full support, this inequality holds with probability one if and only if $\kappa_{1}(Z)=\beta_{0}+\beta_{1} \bar{Z}$.

Proof: Fix a continuation value as above, and let $\kappa_{1}(Z)=\beta_{0}+\beta_{1} \bar{Z}$. We see that

$$
\kappa_{2}(Z)=-\bar{Z}-\frac{\kappa_{1}(Z)}{2 \kappa_{0} n^{2}}
$$

\footnotetext{
${ }^{34}$ Likewise, this is also a Bayesian Nash equilibrium of the incomplete-information game, after specifying beliefs about other traders' inventories.
} 
and thus the transfer to trader $i$ is

$$
\begin{aligned}
& \kappa_{0}\left(n \kappa_{2}(Z)+\sum_{j=1}^{n} \hat{z}^{j}\right)^{2}+\kappa_{1}(Z)\left(\hat{z}^{i}+\kappa_{2}(Z)\right)+\frac{\kappa_{1}^{2}(Z)}{4 \kappa_{0} n^{2}} \\
& =\kappa_{0}\left(-Z-\frac{\kappa_{1}(Z)}{2 \kappa_{0} n}+Z\right)^{2}+\kappa_{1}(Z)\left(z_{0}^{i}-\bar{Z}-\frac{\kappa_{1}(Z)}{2 \kappa_{0} n^{2}}\right)+\frac{\kappa_{1}^{2}(Z)}{4 \kappa_{0} n^{2}} \\
& =\frac{\kappa_{1}^{2}(Z)}{4 \kappa_{0} n^{2}}+\kappa_{1}(Z)\left(z_{0}^{i}-\bar{Z}\right)-\frac{\kappa_{1}^{2}(Z)}{2 \kappa_{0} n^{2}}+\frac{\kappa_{1}^{2}(Z)}{4 \kappa_{0} n^{2}} \\
& =\kappa_{1}(Z)\left(z_{0}^{i}-\bar{Z}\right) \\
& =\left(\beta_{0}+\beta_{1} \bar{Z}\right)\left(z_{0}^{i}-\bar{Z}\right) .
\end{aligned}
$$

From Proposition 3, trader $i$ has the equilibrium post-reallocation inventory $\bar{Z}$. The equilibrium utility of trader $i$ is then simply

$$
u^{i}(\bar{Z})+\kappa_{1}(Z)\left(z_{0}^{i}-\bar{Z}\right)=u^{i}(\bar{Z})+\left(\beta_{0}+\beta_{1} \bar{Z}\right)\left(z_{0}^{i}-\bar{Z}\right) .
$$

Comparing this with $V^{i}\left(z_{0}^{i}, Z\right)$, the result follows from the fact that $K>0$.

For the uniqueness of $\kappa_{1}(\cdot)$, we note that for the IR condition to hold with probability 1 , by continuity, it must hold in the event that $z_{0}^{i}=\bar{Z}$ for all $i$. In this case, the change in utility for any trader is just the transfer received by that trader. By the definition of the transfers, straightforward algebra shows that for any vector $\hat{z}$ of reports,

$$
\begin{aligned}
\sum_{i=1}^{n} T_{\kappa}^{i}(\hat{z}, Z) & =\sum_{i=1}^{n}\left(\kappa_{0}\left(n \kappa_{2}(Z)+\sum_{j=1}^{n} \hat{z}^{j}\right)^{2}+\kappa_{1}(Z)\left(\hat{z}^{i}+\kappa_{2}(Z)\right)+\frac{\kappa_{1}^{2}(Z)}{4 \kappa_{0} n^{2}}\right) \\
& =-n\left(\sqrt{-\kappa_{0}}\left(n \kappa_{2}(Z)+\sum_{j=1}^{n} \hat{z}^{j}\right)-\frac{\kappa_{1}(Z)}{2 \sqrt{-\kappa_{0}} n}\right)^{2} .
\end{aligned}
$$

Plugging in the choice of $\kappa_{2}(\cdot)$ suggested in Proposition 3 and using the equilibrium truthtelling property that $\hat{z}^{i}=z_{0}^{i}$, we have

$$
\sum_{i=1}^{n} T_{\kappa}^{i}(\hat{z}, Z)=-n\left(\sqrt{-\kappa_{0}} \frac{-\kappa_{1}(Z)+\left(\frac{n-1}{n}\right)\left(\beta_{0}+\beta_{1} \bar{Z}\right)}{2 \kappa_{0}}-\frac{\kappa_{1}(Z)}{2 \sqrt{-\kappa_{0}} n}\right)^{2}
$$

which is nonnegative if and only if $\kappa_{1}(Z)=\beta_{0}+\beta_{1} \bar{Z}$, completing the proof.

In summary, if the aggregate inventory $Z$ is known to all traders and to the size-discovery platform operator, then the budget-balanced mechanism $\mathcal{M}^{\kappa}$ can implement a perfect reallocation in an ex-post individually rational equilibrium. ${ }^{35}$ Proposition 4 also implies that the

\footnotetext{
${ }^{35}$ As remarked to one of us by Romans Pancs, a Vickrey-Clarke-Groves (VCG) pivot mechanism can also implement a perfect reallocation in an ex-post equilibrium in this setting. We focus on these transfers because they are tractable in our dynamic game, and as demonstrated by the results of this appendix, there is no sense in which VCG or the AGV mechanism of Arrow (1979) and d'Aspremont and Gérard-Varet (1979) could improve
} 
equilibrium payoffs do not depend upon the choice of $\kappa_{0}$. For $\kappa_{1}(\cdot)$ and $\kappa_{2}(\cdot)$ as specified in Proposition 4, some algebra shows that the equilibrium cash transfer to trader $i$ is

$$
\kappa_{1}(Z)\left(z_{0}^{i}-\bar{Z}\right)=\left(\beta_{0}+\beta_{1} \bar{Z}\right)\left(z_{0}^{i}-\bar{Z}\right) .
$$

The mechanism designer is thus free to choose any $\kappa_{0}<0$, because the choice of $\kappa_{0}$ has no impact on equilibrium transfers or allocations. Result 4 of Proposition 3 nevertheless indicates the strategy-proofness advantage of the particular choice $\kappa_{0}=-K(n-1) / n^{2}$.

\section{A.3 Relationship with the Vickrey-Clarke-Groves mechanism}

We now show how our mechanism relates to the VCG mechanism. ${ }^{36}$ Since the VCG mechanism is defined in terms of value functions, we use the equilibrium value functions from Proposition 1. We adopt the notation of Fudenberg and Tirole (1991), Chapter 7. In this notation, the type $\theta^{i}$ of trader $i$ is her excess inventory $z_{0}^{i}$. For the purpose of this exercise, we assume the platform designer has inferred the average inventory $\bar{Z}$ from the market-clearing price. Let $J$ denote this inference, and suppose the platform designer has simply replaced $\bar{Z}$ with $J$ in the equilibrium value functions, ignoring the relationship between $J$ and the vector $\theta$ of excess inventories. Then for any decision $x$, the equilibrium value for trader $i$ is

$$
V^{i}(x, \theta)=a_{i}+v J-\frac{\gamma}{r} J^{2}+\left(v-\frac{2 \gamma}{r} J\right)\left(\theta^{i}+x^{i}-J\right)-K\left(\theta^{i}+x^{i}-J\right)^{2} .
$$

The efficient allocation $x^{*}(\theta)$ still gives everyone an allocation of $\theta^{i}+x^{* i}(\theta)=\sum_{i} \theta^{i} / n$, although this is not necessarily equal to $J$. In this case, fixing a trader $j$,

$$
V^{j}\left(x^{*}(\hat{\theta}), \hat{\theta}\right)=a_{j}+v J-\frac{\gamma}{r} J^{2}+\left(v-\frac{2 \gamma}{r} J\right)\left(\frac{\sum_{k} \hat{\theta}^{k}}{n}-J\right)-K\left(\frac{\sum_{k} \hat{\theta}^{k}}{n}-J\right)^{2} .
$$

upon the mechanism outcome.

${ }^{36}$ We thank an anonymous referee for suggesting this analysis. 
Now, fix some $i \neq j$ and rewrite this expression as

$$
\begin{aligned}
& a_{j}+v J-\frac{\gamma}{r} J^{2}+\left(v-\frac{2 \gamma}{r} J\right)\left(\frac{\sum_{k} \hat{\theta}^{k}}{n}-J\right)-K\left(\frac{\sum_{k} \hat{\theta}^{k}}{n}-J\right)^{2} \\
& =\left(v-\frac{2 \gamma}{r} J\right) \frac{\hat{\theta}^{i}}{n}-\frac{K}{n^{2}}\left(\sum_{k} \hat{\theta}^{k}-n J\right)^{2}+f\left(\hat{\theta}^{-i}\right) \\
& =\left(v-\frac{2 \gamma}{r} J\right) \frac{\hat{\theta}^{i}}{n}-\frac{K}{n^{2}}\left(\sum_{k} \hat{\theta}^{k}+n \beta(p)-n \beta(p)-n J\right)^{2}+f\left(\hat{\theta}^{-i}\right) \\
& =\left(v-\frac{2 \gamma}{r} J\right) \frac{\hat{\theta}^{i}}{n}-\frac{K}{n^{2}}\left(\sum_{k} \hat{\theta}^{k}-n \beta(p)\right)^{2}-\frac{2 K}{n^{2}}\left(\sum_{k} \hat{\theta}^{k}-n \beta(p)\right)(n \beta(p)-n J)+f\left(\hat{\theta}^{-i}\right) \\
& =\left(v-\frac{2 \gamma}{r} J\right) \frac{\hat{\theta}^{i}}{n}-\frac{K}{n^{2}}\left(\sum_{k} \hat{\theta}^{k}-n \beta(p)\right)^{2}-\frac{2 K}{n^{2}} \hat{\theta}^{i}(n \beta(p)-n J)+f\left(\hat{\theta}^{-i}\right),
\end{aligned}
$$

where $f\left(\hat{\theta}^{-i}\right)$ collects terms that do not depend on $\hat{\theta}^{i}$ and $\beta(p)$ is any constant that does not depend on $\hat{\theta}^{i}$. It follows that the VCG transfer for trader $i$ is

$$
\sum_{j \neq i} V^{j}\left(x^{*}(\hat{\theta}), \hat{\theta}\right)=-\frac{K(n-1)}{n^{2}}\left(\sum_{k} \hat{\theta}^{k}-n \beta(p)\right)^{2}+\hat{\theta}^{i} \Theta+f\left(\hat{\theta}^{-i}\right),
$$

where

$$
\Theta=\frac{n-1}{n}\left(v-\frac{2 \gamma}{r} J\right)-\frac{2 K(n-1)}{n^{2}}(n \beta(p)-n J) .
$$

Now, in the equilibrium of Proposition 1, we know that $p=v-(2 \gamma / r) J$, so plugging this in,

$$
\Theta=\frac{n-1}{n} p-\frac{2 K(n-1)}{n^{2}}\left(n \beta(p)-n \frac{r(v-p)}{2 \gamma}\right) .
$$

Plug in $\kappa_{0}=-K(n-1) /\left(n^{2}\right)$ and

$$
\beta(p)=\frac{r v}{2 \gamma}+p\left(\frac{-r}{2 \gamma}+\frac{1}{2 \kappa_{0} n^{2}}\right)
$$

to see that $\Theta=p$, and thus the VCG mechanism coincides with our mechanism at the strategyproof choice. Our mechanism simply sets $f\left(\hat{\theta}^{-i}\right)$ to satisfy ex-post individual rationality and budget balance. Again, this is possible because the platform designer has already inferred the aggregate inventory. This shows that our linear-quadratic mechanism, which works for any negative constant $\kappa_{0}$, coincides with the VCG mechanism associated with the equilibrium value functions for a particular $\kappa_{0}$. When we say our mechanism is strategy proof, we mean it in the well-known sense that this VCG mechanism is strategy proof, given the platform designer has 
the correct inference $J$.

\section{B Proof of Proposition 1}

The proof of the main result of the paper, Proposition 1, proceeds in steps.

First, we characterize all of the possible equilibrium value functions. We prove the exchange demand process implied by demand coefficients $(a, b, c)$ is admissible if and only if $r+\lambda-2 c>$ 0 . We will show that $c<0$ in all of our equilibria, implying admissibility is satisfied. We then calculate closed-form solutions for the value functions implied by any candidate demand coefficients $(a, b, c)$ satisfying the admissibility condition.

Next, we use the HJB equation for the stochastic control problem faced by trader $i$ to narrow the set of possible equilibria. The candidate value functions are all linear quadratic and thus twice continuously differentiable, so this HJB is a necessary condition for the candidate strategies to be optimal. Subsection B.3 of this appendix is a lengthy derivation of the explicit coefficients of the candidate value functions $V^{i}$ and the equilibrium demand coefficients that are consistent with the HJB equation. We show there are two candidate equilibria which satisfy the HJB equation, one of which is the candidate value function $V^{i}$ of (9) with the claimed equilibrium demand coefficients $(a, b, c)$ of Proposition 1. Subsection B.3 also proves the monotonicity properties related to $\lambda$ and other properties stated by Proposition 1 .

Finally, we perform a standard martingale-based verification argument of optimality of the candidate optimal strategy for trader $i$, assuming all other traders adopt their candidate optimal strategies. For this, we show that the candidate value $V^{i}\left(z_{0}^{i}, Z_{0}\right)$ is equal to the expected total payoff of the candidate optimal strategy, and is greater than or equal to the expected payoff of any admissible strategy. This implies that the candidate optimal strategy is in fact optimal, and completes the proof of equilibrium.

\section{B.1 Equilibrium value functions}

In this section, we calculate closed-form solutions for the value functions that result from all traders truthfully reporting and using an affine demand process with coefficients $(a, b, c)$, as they must in any equilibrium by definition. We ignore until the next section whether such strategies are optimal.

\section{B.1.1 A technical lemma}

In this section we prove a technical lemma that will be useful in all subsequent proofs.

Lemma 2. Let $c \neq 0$ be an arbitrary constant, and let $\bar{Z}_{t}$ and $\sigma_{Z}^{2}$ be defined as in the text. Then, for any $t$,

$$
\mathbb{E}\left[\int_{0}^{t} e^{-c s} \bar{Z}_{s} d s\right]=\bar{Z}_{0} \frac{1-e^{-c t}}{c}
$$

and

$$
\mathbb{E}\left[\left(\int_{0}^{t} e^{-c s} \bar{Z}_{s} d s\right)^{2}\right]=\frac{\left(1-e^{-c t}\right)^{2}}{c^{2}} \bar{Z}_{0}^{2}+\frac{\sigma_{Z}^{2}}{n^{2}} \frac{e^{-2 c t}\left(2 c t-4 e^{c t}+e^{2 c t}+3\right)}{2 c^{3}}
$$


As $c \rightarrow 0$, these expectations converge to the expectations of the limiting integrands, and in particular

$$
\mathbb{E}\left[\left(\int_{0}^{t} \bar{Z}_{s} d s\right)^{2}\right]=\bar{Z}_{0}^{2} t^{2}+\frac{\sigma_{Z}^{2}}{n^{2}} \frac{t^{3}}{3} .
$$

Proof: Fixing $s$, because $\mathbb{E}\left[\left(\bar{Z}_{s}\right)^{2}\right]=\bar{Z}_{0}^{2}+\left(\sigma_{Z}^{2} / n^{2}\right) s$ by assumption, we can apply Hölder's inequality to find that

$$
\mathbb{E}\left[\left|e^{-c s} \bar{Z}_{s}\right|\right] \leq e^{-c s} \sqrt{\mathbb{E}\left[\left(\bar{Z}_{s}\right)^{2}\right]}=e^{-c s} \sqrt{\bar{Z}_{0}^{2}+\frac{\sigma_{Z}^{2}}{n^{2}} s} .
$$

It follows that, for any $t$,

$$
\int_{0}^{t} \mathbb{E}\left[\left|e^{-c s} \bar{Z}_{s}\right|\right] d s \leq \int_{0}^{t} e^{-c s} \sqrt{\bar{Z}_{0}^{2}+\frac{\sigma_{Z}^{2}}{n^{2}}} s d s<\infty .
$$

We may thus apply the Fubini-Tonelli theorem to write that

$$
\mathbb{E}\left[\int_{0}^{t} e^{-c s} \bar{Z}_{s} d s\right]=\int_{0}^{t} \mathbb{E}\left[e^{-c s} \bar{Z}_{s}\right] d s=\bar{Z}_{0} \int_{0}^{t} e^{-c s} d s=\bar{Z}_{0} \frac{1-e^{-c t}}{c}
$$

where we have used the fact that, from the definition of $H_{t}$, we have $\mathbb{E}\left[\bar{Z}_{s}\right]=\bar{Z}_{0}$. Henceforth, for brevity we refer to this as the "Hölder's inequality and Fubini-Tonelli theorem argument."

Now, define $W_{t}=\int_{0}^{t} e^{-c s} \bar{Z}_{s} d s$. By Ito's lemma,

$$
W_{t}^{2}=2 \int_{0}^{t} W_{s} e^{-c s} \bar{Z}_{s} d s=2 \int_{0}^{t} \int_{0}^{s} e^{-c s} \bar{Z}_{s} e^{-c u} \bar{Z}_{u} d u d s .
$$

By the Lévy property, $\mathbb{E}\left[\bar{Z}_{u}\left(\bar{Z}_{s}-\bar{Z}_{u}\right)\right]=0$. An application of the "Hölder's inequality and Fubini-Tonelli theorem argument" implies that

$$
\begin{aligned}
\mathbb{E}\left[\int_{0}^{t} \int_{0}^{s} e^{-c s} \bar{Z}_{s} e^{-c u} \bar{Z}_{u} d u d s\right] & =\int_{0}^{t} \int_{0}^{s} \mathbb{E}\left[e^{-c s} \bar{Z}_{s} e^{-c u} \bar{Z}_{u}\right] d u d s \\
& =\int_{0}^{t} \int_{0}^{s} \mathbb{E}\left[e^{-c s} e^{-c u}\left(\bar{Z}_{s}-\bar{Z}_{u}+\bar{Z}_{u}\right) \bar{Z}_{u}\right] d u d s \\
& =\int_{0}^{t} \int_{0}^{s} \mathbb{E}\left[e^{-c s} e^{-c u} \bar{Z}_{u}^{2}\right] d u d s \\
& =\int_{0}^{t} \int_{0}^{s} e^{-c s} e^{-c u}\left(\bar{Z}_{0}^{2}+\frac{\sigma_{Z}^{2}}{n^{2}} u\right) d u d s \\
& =\frac{\left(1-e^{-c t}\right)^{2}}{2 c^{2}} \bar{Z}_{0}^{2}+\frac{\sigma_{Z}^{2}}{n^{2}} \frac{e^{-2 c t}\left(2 c t-4 e^{c t}+e^{2 c t}+3\right)}{4 c^{3}} .
\end{aligned}
$$

Finally, starting at the penultimate line of the above system and plugging in $c=0$, we arrive at

$$
\mathbb{E}\left[\left(\int_{0}^{t} \bar{Z}_{s} d s\right)^{2}\right]=\bar{Z}_{0}^{2} t^{2}+\frac{\sigma_{Z}^{2}}{n^{2}} \frac{t^{3}}{3} .
$$


This proves the technical lemma. We now address admissibility.

\section{B.1.2 Admissibility}

In this section, we show that if there were a symmetric affine equilibrium with $2 c \geq r+\lambda$, then one trader would be using an inadmissible strategy, meaning that the value achieved in the stochastic control problem (8) would be negative infinity or undefined. In order to see this, fix candidate demand coefficients $(a, b, c)$. Then each trader demands the asset at the flow rate $D_{t}=a+b \phi_{t}+c z_{t}^{i}$, so the market-clearing price must be

$$
\phi_{t}=\frac{a+c \bar{Z}_{t}}{-b}
$$

Plugging this price back into trader demands, we can write

$$
D_{t}=c\left(z-\bar{Z}_{t}\right)
$$

Let $T_{1}$ denote the minimum of $\mathcal{T}$ and the first jump time of $N$. It follows that if all traders follow this strategy, the inventory of trader $i$ at any time $t<T_{1}$ is

$$
z_{t}^{i}=z_{0}^{i}+c \int_{0}^{t}\left(z_{s}^{i}-\bar{Z}_{s}\right) d s+H_{t}^{i}
$$

Applying Ito's lemma for semimartingales to $e^{-c t} z_{t}^{i}$, and multiplying both sides by $e^{c t}$, one can show ${ }^{37}$ that

$$
z_{t}^{i}=e^{c t} z_{0}^{i}-e^{c t} c \int_{0}^{t} e^{-c s} \bar{Z}_{s} d s+e^{c t} \int_{0}^{t} e^{-c s} d H_{s}^{i}
$$

We now show that $\mathbb{E}\left[\int_{0}^{T_{1}}\left(z_{s}^{i}\right)^{2} d s\right]$ is finite if and only if $2 c<r+\lambda$. We first must compute a few quantities. Fix a time $t<T_{1}$. Because $e^{-c s}$ is square integrable, the last term in the expression for $z_{t}^{i}$ is a martingale, so by Lemma 2 ,

$$
\mathbb{E}\left(z_{t}^{i}\right)=e^{c t} z_{0}^{i}+\bar{Z}_{0}\left(1-e^{c t}\right) .
$$

Next, we evaluate

$$
\mathbb{E}\left[\int_{0}^{t} e^{-c s} \bar{Z}_{s} d s \int_{0}^{t} e^{-c s} d H_{s}^{i}\right]
$$

Let $A_{t} \equiv \int_{0}^{t} e^{-c s} \bar{Z}_{s} d s$ and $B_{t} \equiv \int_{0}^{t} e^{-c s} d H_{s}^{i}$. Note that $[A, B]_{t}=0$ since $A$ is a continuous finite-variation process, so by Ito's lemma for semimartingales,

$$
d\left(A_{t} B_{t}\right)=A_{t} d B_{t}+B_{t} d A_{t}=A_{t} e^{-c t} d H_{t}^{i}+B_{t} e^{-c t} \bar{Z}_{t} d t
$$

or

$$
\int_{0}^{t} e^{-c s} \bar{Z}_{s} d s \int_{0}^{t} e^{-c s} d H_{s}^{i}=\int_{0}^{t} e^{-c s} \int_{0}^{s} e^{-c u} \bar{Z}_{u} d u d H_{s}^{i}+\int_{0}^{t} e^{-c s} \bar{Z}_{s} \int_{0}^{s} e^{-c u} d H_{u}^{i} d s .
$$

\footnotetext{
${ }^{37}$ This is exactly the derivation of the solution of the Ornstein-Uhlenbeck process.
} 
Since $H_{t}^{i}$ is a martingale and $\int_{0}^{s} e^{-c u} \bar{Z}_{u} d u$ is square integrable by Lemma 2, we have

$$
\mathbb{E}\left[\int_{0}^{t} e^{-c s} \bar{Z}_{s} d s \int_{0}^{t} e^{-c s} d H_{s}^{i}\right]=\mathbb{E}\left[\int_{0}^{t} e^{-c s} \bar{Z}_{s} \int_{0}^{s} e^{-c u} d H_{u}^{i} d s\right] .
$$

Applying the "Hölder's inequality and Fubini-Tonelli theorem argument" this expectation is

$$
\int_{0}^{t} e^{-c s} \mathbb{E}\left[\bar{Z}_{s} \int_{0}^{s} e^{-c u} d H_{u}^{i}\right] d s
$$

where, by another application of Itô's formula for semimartingales and a well known result on the quadratic covariation of semimartingales,

$$
\bar{Z}_{t} \int_{0}^{t} e^{-c u} d H_{u}^{i}=\int_{0}^{t}\left(\int_{0}^{s} e^{-c u} d H_{u}^{i}\right) d \bar{Z}_{s}+\int_{0}^{t} \bar{Z}_{s} e^{-c s} d H_{s}^{i}+\int_{0}^{t} e^{-c u} d\left[H^{i}, \bar{Z}\right]_{u} .
$$

Since the integrands $\int_{0}^{s} e^{-c u} d H_{u}^{i}$ and $\bar{Z}_{s} e^{-c s}$ are square integrable, we have

$$
\mathbb{E}\left[\bar{Z}_{t} \int_{0}^{t} e^{-c u} d H_{u}^{i}\right]=\mathbb{E}\left[\int_{0}^{t} e^{-c u} d\left[H^{i}, \bar{Z}\right]_{u}\right]=\int_{0}^{t} e^{-c u} \frac{\rho^{i}}{n} d u=\frac{\rho^{i}}{n c}\left(1-e^{-c t}\right) .
$$

Putting this together,

$$
\mathbb{E}\left[\int_{0}^{t} e^{-c s} \bar{Z}_{s} d s \int_{0}^{t} e^{-c s} d H_{s}^{i}\right]=\int_{0}^{t} e^{-c s} \frac{\rho^{i}}{n c}\left(1-e^{-c s}\right) d s=\frac{\rho^{i}}{2 n c^{2}}\left(1-e^{-c t}\right)^{2} .
$$

Next, applying Itô isometry for martingales, and recalling that $\left[H^{i}, H^{i}\right]_{t}=\sigma_{i}^{2} t$ because $H^{i}$ is square integrable, we have

$$
\mathbb{E}\left[\left(\int_{0}^{t} e^{-c s} d H_{s}^{i}\right)^{2}\right]=\int_{0}^{t} e^{-2 c s} \sigma_{i}^{2} d s=\frac{-\sigma_{i}^{2}}{2 c}\left(e^{-2 c t}-1\right) .
$$

Combining these pieces,

$$
\begin{aligned}
\mathbb{E}\left[\left(z_{t}^{i}\right)^{2}\right]= & e^{2 c t}\left(\mathbb{E}\left[\left(z_{0}^{i}-c \int_{0}^{t} e^{-c s} \bar{Z}_{s} d s\right)^{2}\right]+\mathbb{E}\left[\left(\int_{0}^{t} e^{-c s} d H_{s}^{i}\right)^{2}\right]\right) \\
& +e^{2 c t} \mathbb{E}\left[\left(z_{0}^{i}-c \int_{0}^{t} e^{-c s} \bar{Z}_{s} d s\right)\left(\int_{0}^{t} e^{-c s} d H_{s}^{i}\right)\right] \\
= & e^{2 c t}\left(z_{0}^{i}\right)^{2}+2 e^{c t} z_{0}^{i} \bar{Z}_{0}\left(1-e^{c t}\right)+\left(1-e^{c t}\right)^{2} \bar{Z}_{0}^{2}+\frac{\sigma_{Z}^{2}}{n^{2}} \frac{\left(2 c t-4 e^{c t}+e^{2 c t}+3\right)}{2 c} \\
& +e^{2 c t}\left(\frac{-\sigma_{i}^{2}}{2 c}\left(e^{-2 c t}-1\right)\right)+e^{2 c t}\left(\frac{\rho^{i}}{2 n c^{2}}\left(1-e^{-c t}\right)^{2}\right) .
\end{aligned}
$$


Applying the independence of $\mathcal{T}, N_{t}$ and $H_{t}^{i}$, as well as Tonelli's theorem, we have

$$
\begin{aligned}
\mathbb{E}\left[\int_{0}^{T_{1}}\left(z_{s}^{i}\right)^{2} d s\right] & =\int_{0}^{\infty}(r+\lambda) e^{-(r+\lambda) t} \int_{0}^{t} \mathbb{E}\left[\left(z_{s}^{i}\right)^{2}\right] d s d t \\
& \leq \int_{0}^{\infty} \int_{0}^{t} \mathbb{E}\left[(r+\lambda) e^{-(r+\lambda) s}\left(z_{s}^{i}\right)^{2}\right] d s d t
\end{aligned}
$$

where we have used the fact that $T_{1}$ is distributed exponentially with parameter $r+\lambda$. From (41), we see that this quantity is finite if and only if $2 c<r+\lambda$. This is true regardless of $z_{0}^{i}$. By a straightforward application of monotone convergence, as long as $2 c<r+\lambda$, this implies that

$$
\mathbb{E}\left[\int_{0}^{\mathcal{T}}\left(z_{s}^{i}\right)^{2} d s\right]=\mathbb{E}\left[\lim _{n \rightarrow \infty} \int_{0}^{T_{n}}\left(z_{s}^{i}\right)^{2} d s\right]=\lim _{n \rightarrow \infty} \mathbb{E}\left[\int_{0}^{T_{n}}\left(z_{s}^{i}\right)^{2} d s\right]<\infty .
$$

We have thus shown that $2 c<r+\lambda$ is a necessary condition for admissibility, and it is a sufficient condition for the expected holding costs to be finite. It turns out this is sufficient for the value function to be well defined, as we prove in the next section, implying it is a sufficient condition for admissibility.

\section{B.1.3 Linear-quadratic form of the value function}

We are now ready to characterize the set of value functions that could possibly be consistent with an equilibrium. Fix symmetric affine equilibrium demand coefficients $(a, b, c)$. As above, the associated equilibrium market-clearing price $\phi_{t}$ is

$$
\phi_{t}=\frac{a+c \bar{Z}_{t}}{-b}
$$

and thus $a+b \phi_{t}+c z_{t}^{i}=c\left(z_{t}^{i}-\bar{Z}_{t}\right)$. Thus, the trading costs paid by trader $i$ in an equilibrium with demand coefficients $(a, b, c)$ is

$$
\int_{0}^{\mathcal{T}} c\left(z_{s}^{i}-\bar{Z}_{s}\right)\left(\frac{a+c \bar{Z}_{s}}{-b}\right) d s .
$$

Recall that the cash transfer of trader $i$ at a given $(\mu, p) \in \mathbb{R}^{n} \times \mathbb{R}$ is

$$
T_{Q}^{i}(\mu, p)=p \mu^{i}+\kappa_{0}\left(-n \beta(p)+\sum_{j=1}^{n} \mu^{j}\right)^{2}-p \beta(p)+\frac{p^{2}}{4 \kappa_{0} n^{2}},
$$

where

$$
\beta(p)=\frac{r v}{2 \gamma}+p\left(\frac{-r}{2 \gamma}+\frac{1}{2 \kappa_{0} n^{2}}\right) .
$$

Plugging in $\mu^{j}=z_{t}^{j}$ for all $j$ and $p=\left(a+c \bar{Z}_{t}\right)(-b)$, we see that in equilibrium the transfer takes the form

$$
R_{0}+R_{1} Z_{t}+R_{2} Z_{t}^{2}+R_{3} Z_{t} z_{t}^{i}+R_{4} z_{t}^{i}
$$


for constants $R_{0}$ through $R_{4}$ that depend on $\kappa_{0}, a, b, c$. These calculations motivate the following lemma, which we use in the proof of Proposition 1 as well as our extensions, that gives the closed-form solution for the value function in any candidate equilibrium.

Lemma 3. Fix any exchange demand-function coefficients $(a, b, c)$ with $2 c<r+\lambda$ and transfer coefficients $R_{0}-R_{4}$. Let

$$
z_{t}^{i}=z_{0}^{i}+\int_{0}^{t} c\left(z_{s}^{i}-\bar{Z}_{s}\right) d s+H_{t}^{i}+\int_{0}^{\mathcal{T}}\left(\bar{Z}_{s}-z_{s}^{i}\right) d N_{s}
$$

denote the corresponding equilibrium inventory process for trader $i$, and let

$$
T^{R}(z, Z)=R_{0}+R_{1} Z+R_{2} Z^{2}+R_{3} Z z^{i}+R_{4} z^{i}
$$

denote the corresponding equilibrium cash transfers. Then

$$
\mathbb{E}\left[\pi z_{\mathcal{T}}^{i}+\int_{0}^{\mathcal{T}}\left(-\gamma\left(z_{s}^{i}\right)^{2}-c\left(z_{s}^{i}-\bar{Z}_{s}\right)\left(\frac{a+c \bar{Z}_{s}}{-b}\right) d s\right)+\int_{0}^{\mathcal{T}} T^{R}\left(z_{s}^{i}, Z_{s}\right) d N_{s}\right]=V\left(z_{0}^{i}, Z_{0}\right),
$$

where

$$
V(z, Z)=\alpha_{0}^{i}+\alpha_{1} z+\alpha_{2} \bar{Z}+\alpha_{3} z^{2}+\alpha_{4} \bar{Z}^{2}+\alpha_{5} z \bar{Z}
$$

and

$$
\begin{aligned}
& \alpha_{3}=\frac{-\gamma}{r+\lambda-2 c} \\
& \alpha_{5}=\frac{1}{r+\lambda-c}\left(\frac{c^{2}}{b}-2 \alpha_{3} c+\lambda n R_{3}\right) \\
& \alpha_{4}=\frac{1}{r}\left(\frac{c^{2}}{-b}+(\lambda-c) \alpha_{5}+\lambda \alpha_{3}+\lambda n^{2} R_{2}\right) \\
& \alpha_{1}=\frac{1}{r+\lambda-c}\left(r v+\frac{a c}{b}+\lambda R_{4}\right) \\
& \alpha_{2}=\frac{1}{r}\left(\frac{c a}{-b}+(\lambda-c) \alpha_{1}+\lambda n R_{1}\right) \\
& \alpha_{0}^{i}=\frac{1}{r}\left(\alpha_{3} \sigma_{i}^{2}+\alpha_{4} \frac{\sigma_{Z}^{2}}{n^{2}}+\alpha_{5} \frac{\rho^{i}}{n}+\lambda R_{0}\right) .
\end{aligned}
$$

Proof: Given the $\alpha$ coefficients, we have

$$
\begin{aligned}
& (r+\lambda)\left(\alpha_{0}^{i}+\alpha_{1} z+\alpha_{2} \bar{Z}+\alpha_{3} z^{2}+\alpha_{4} \bar{Z}^{2}+\alpha_{5} z \bar{Z}\right) \\
& =r v z-\gamma z^{2}+\alpha_{4} \frac{\sigma_{Z}^{2}}{n^{2}}+\alpha_{3} \sigma_{i}^{2}+\alpha_{5} \frac{\rho^{i}}{n}-c(z-\bar{Z}) \frac{a+c \bar{Z}}{-b} \\
& \quad+c(z-\bar{Z})\left(\alpha_{1}+2 \alpha_{3} z+\alpha_{5} \bar{Z}\right)+\lambda\left(\alpha_{0}^{i}+\alpha_{1} \bar{Z}+\alpha_{2} \bar{Z}+\alpha_{3} \bar{Z}^{2}\right. \\
& \left.\quad+\alpha_{4} \bar{Z}^{2}+\alpha_{5} \bar{Z}^{2}+R_{0}+R_{1} Z+R_{2} Z^{2}+R_{3} Z z+R_{4} z\right) .
\end{aligned}
$$


Let $Y_{t}=1_{\{\mathcal{T} \leq t\}}$ and $V(z, Z)$ be defined as above. Let

$$
X=\left[\begin{array}{c}
z_{t}^{i} \\
Z_{t} \\
Y_{t}
\end{array}\right]
$$

and $U(X)=U(z, Z, Y)=(1-Y) V(z, Z)+Y v z$. Then, by Ito's lemma for semimartingales, for any $t$, we have

$$
\begin{aligned}
& U\left(X_{t}\right)-U\left(X_{0}\right)=\int_{0+}^{t}\left(1-Y_{s-}\right) V_{z}\left(z_{s-}^{i}, Z_{s-}\right)+Y_{s-} v d z_{s}^{i}+\int_{0+}^{t}\left(1-Y_{s-}\right) V_{Z}\left(z_{s-}^{i}, Z_{s-}\right) d Z_{s} \\
&+ \frac{1}{2} \int_{0+}^{t}\left(1-Y_{s-}\right) V_{z z}\left(z_{s-}^{i}, Z_{s-}\right) d\left[z^{i}, z^{i}\right]_{s}^{c}+\frac{1}{2} \int_{0+}^{t}\left(1-Y_{s-}\right) V_{Z Z}\left(z_{s-}^{i}, Z_{s-}\right) d[Z, Z]_{s}^{c} \\
&+\int_{0+}^{t}\left(1-Y_{s-}\right) V_{z Z}\left(z_{s-}^{i}, Z_{s-}\right) d\left[z^{i}, Z\right]_{s}^{c} \\
&+\sum_{0 \leq s \leq t} U\left(X_{s}\right)-U\left(X_{s-}\right)-\left[\left(1-Y_{s-}\right) V_{z}\left(z_{s-}^{i}, Z_{s}\right)+Y_{s-} v\right] \Delta z_{s}^{i} \\
&-\sum_{0 \leq s \leq t}\left(1-Y_{s-}\right) V_{Z}\left(z_{s-}^{i}, Z_{s}\right) \Delta Z_{s}
\end{aligned}
$$

where we have used the fact that

$$
\int_{0+}^{t} \frac{\partial}{\partial Y} U\left(z_{s-}^{i}, Y_{s-}\right) d Y_{s}=\sum_{0 \leq s \leq t} \frac{\partial}{\partial Y} U\left(z_{s-}^{i}, Y_{s-}\right) \Delta Y_{s}
$$

and the fact that $\left[z^{i}, Y\right]^{c}=[Z, Y]^{c}=[Y, Y]^{c}=0$.

Now, we note that

$$
\begin{gathered}
V\left(z_{s}^{i}, Z_{s}\right)-V\left(z_{s-}^{i}, Z_{s-}\right)=\alpha_{1} \Delta z_{s}^{i}+\alpha_{2} \frac{\Delta Z_{s}}{n}+\alpha_{4}\left(\frac{\Delta Z_{s}}{n}\right)^{2}+2 \alpha_{4} \frac{Z_{s-} \Delta Z_{s}}{n^{2}} \\
+\alpha_{3}\left(\Delta z_{s}^{i}\right)^{2}+2 \alpha_{3} z_{s-}^{i} \Delta z_{s}^{i}+\alpha_{5} z_{s-}^{i} \frac{\Delta Z_{s}}{n} \\
+\alpha_{5} \bar{Z}_{s-} \Delta z_{s}^{i}+\alpha_{5} \frac{\Delta Z_{s}}{n} \Delta z_{s}^{i}
\end{gathered}
$$

while

$$
\begin{aligned}
V_{Z}\left(z_{s-}^{i}, Z_{s-}\right) \Delta Z_{s} & =\frac{\Delta Z_{s}}{n}\left(\alpha_{2}+\alpha_{5} z_{s-}^{i}+2 \alpha_{4} \bar{Z}_{s-}\right) \\
V_{z}\left(z_{s-}^{i}, Z_{s-}\right) \Delta z_{s}^{i} & =\Delta z_{s}^{i}\left(\alpha_{1}+\alpha_{5} \bar{Z}_{s-}+2 \alpha_{3} z_{s-}^{i}\right) .
\end{aligned}
$$

Thus, the total contribution to the sum in (48) from jumps in $z_{s}^{i}$ or $Z_{s}$ is given by

$$
\left(1-Y_{s-}\right)\left(\alpha_{4}\left(\frac{\Delta Z_{s}}{n}\right)^{2}+\alpha_{3}\left(\Delta z_{s}^{i}\right)^{2}+\alpha_{5} \frac{\Delta Z_{s}}{n} \Delta z_{s}^{i}\right)
$$


because the term $-Y_{s-} v \Delta z_{s}^{i}$ is cancelled by the same term in $U\left(X_{s}\right)-U\left(X_{s-}\right)$.

We note that jumps in $z^{i}$ arise from jumps in both $H^{i}$ and $N$. By independence, $\Delta N \Delta H^{i}=$ $\Delta N \Delta Z=\Delta Y \Delta Z=\Delta Y \Delta z^{i}=0$ with probability 1 . In summary, we can write the sum as

$$
\begin{aligned}
& \sum_{0 \leq s \leq t} \Delta Y_{s}\left(v z_{s-}^{i}-V\left(z_{s-}^{i}, Z_{s-}\right)\right) \\
& \quad+\left(1-Y_{s-}\right)\left(\alpha_{4}\left(\frac{\Delta Z_{s}}{n}\right)^{2}+\alpha_{3}\left(\Delta H_{s}^{i}\right)^{2}+\alpha_{3} \Delta N_{s}\left(z_{s-}^{i}-\bar{Z}_{s-}\right)^{2}+\alpha_{5} \frac{\Delta Z_{s}}{n} \Delta H_{s}^{i}\right) .
\end{aligned}
$$

It will be convenient to write

$$
\begin{aligned}
\sum_{0 \leq s \leq t}\left(1-Y_{s-}\right)\left(\alpha_{3} \Delta N_{s}\left(z_{s-}^{i}-\bar{Z}_{s-}\right)^{2}\right)= & \int_{0}^{t}\left(1-Y_{s-}\right) \alpha_{3}\left(z_{s-}^{i}-\bar{Z}_{s-}\right)^{2} d N_{s} \\
= & \int_{0}^{t}\left(1-Y_{s-}\right) \alpha_{3}\left(z_{s-}^{i}-\bar{Z}_{s-}\right)^{2}\left(d N_{s}-\lambda d s\right) \\
& +\int_{0}^{t}\left(1-Y_{s-}\right) \lambda \alpha_{3}\left(z_{s-}^{i}-\bar{Z}_{s-}\right)^{2} d s
\end{aligned}
$$

Finally, we note that

$$
\begin{aligned}
\int_{0+}^{t} V_{z}\left(z_{s-}^{i}, Z_{s-}\right) d z_{s}^{i}= & \int_{0+}^{t}\left(\alpha_{1}+\alpha_{5} \bar{Z}_{s-}+2 \alpha_{3} z_{s-}^{i}\right) d z_{s}^{i} \\
= & \int_{0+}^{t}\left(\alpha_{1}+\alpha_{5} \bar{Z}_{s-}+2 \alpha_{3} z_{s-}^{i}\right)\left((c-\lambda)\left(z_{s}^{i}-\bar{Z}_{s}\right)\right) d s \\
& +\int_{0+}^{t}\left(\alpha_{1}+\alpha_{5} \bar{Z}_{s-}+2 \alpha_{3} z_{s-}^{i}\right) d H_{s}^{i} \\
& +\int_{0+}^{t}\left(\alpha_{1}+\alpha_{5} \bar{Z}_{s-}+2 \alpha_{3} z_{s-}^{i}\right)\left(\bar{Z}_{s}-z_{s-}^{i}\right) d\left(N_{s}-\lambda d s\right) .
\end{aligned}
$$

We let

$$
\begin{aligned}
\chi_{s}=c & \left(z_{s}^{i}-\bar{Z}_{s}\right)\left(\alpha_{1}+\alpha_{5} \bar{Z}_{s-}+2 \alpha_{3} z_{s-}^{i}\right)+\alpha_{4} \frac{\sigma_{Z}^{2}}{n^{2}}+\alpha_{3} \sigma_{i}^{2}+\alpha_{5} \frac{\rho^{i}}{n} \\
& -\lambda\left(z_{s}^{i}-\bar{Z}_{s}\right)\left(\alpha_{1}+\alpha_{5} \bar{Z}_{s-}+\alpha_{3}\left(z_{s-}^{i}+\bar{Z}_{s-}\right)\right)+r\left(v z_{s}^{i}-V\left(z_{s}^{i}, Z_{s}\right)\right) .
\end{aligned}
$$


Plugging in $V_{Z Z}=2 \alpha_{4} / n^{2}, V_{z z}=2 \alpha_{3}, V_{z Z}=\alpha_{5} / n$, and evaluating (48) at $t=\mathcal{T}$, we can write

$$
\begin{aligned}
U\left(X_{\mathcal{T}}\right)-U\left(X_{0}\right)=\int_{0+}^{\mathcal{T}} \chi_{s} d s+\int_{0+}^{\mathcal{T}}\left(\alpha_{1}+\alpha_{5} \bar{Z}_{s-}+2 \alpha_{3} z_{s-}^{i}\right) d H_{s}^{i} \\
+\int_{0+}^{\mathcal{T}}\left(\alpha_{1}+\alpha_{5} \bar{Z}_{s-}+2 \alpha_{3} z_{s-}^{i}\right)\left(\bar{Z}_{s}-z_{s}^{i}\right) d\left(N_{s}-\lambda d s\right) \\
+\int_{0}^{\mathcal{T}} \alpha_{3}\left(z_{s-}^{i}-\bar{Z}_{s-}\right)^{2}\left(d N_{s}-\lambda d s\right)+\int_{0+}^{\mathcal{T}} \frac{1}{n}\left(\alpha_{2}+\alpha_{5} z_{s-}^{i}+2 \alpha_{4} \bar{Z}_{s-}\right) d Z_{s} \\
+\alpha_{3}\left(-\sigma_{i}^{2} \mathcal{T}+\int_{0+}^{\mathcal{T}} d\left[H^{i}, H^{i}\right]_{s}^{c}+\sum_{0 \leq s \leq \mathcal{T}}\left(\Delta H_{s}^{i}\right)^{2}\right) \\
+\frac{\alpha_{4}}{n^{2}}\left(-\sigma_{Z}^{2} \mathcal{T}+\int_{0+}^{\mathcal{T}} d[Z, Z]_{s}^{c}+\sum_{0 \leq s \leq \mathcal{T}}\left(\Delta Z_{s}\right)^{2}\right) \\
+\frac{\alpha_{5}}{n}\left(-\rho^{i} \mathcal{T}+\int_{0+}^{\mathcal{T}} d\left[Z, H^{i}\right]_{s}^{c}+\sum_{0 \leq s \leq \mathcal{T}}\left(\Delta Z_{s} \Delta H_{s}^{i}\right)\right) \\
+\int_{0}^{\mathcal{T}}\left(v z_{s-}^{i}-V\left(z_{s-}^{i}, Z_{s-}\right)\right)\left(d Y_{s}-r d s\right)
\end{aligned}
$$

where we have replaced $Y_{s-}=0$ for $s \leq \mathcal{T}$, by definition. Also, we have used the fact that $\left[z^{i}, z^{i}\right]^{c}=\left[H^{i}, H^{i}\right]^{c}$ and $\left[z^{i}, Z\right]^{c}=\left[H^{i}, Z\right]^{c}$, since $z^{i}$ is the sum of $H_{t}^{i}$ and a finite-variation process that is a quadratic pure-jump semimartingale. (See (Protter, 2005).)

For any deterministic $\mathcal{T}$, it is well known from the theory of Lévy processes that

$$
\begin{aligned}
\mathbb{E}\left[\left(-\sigma_{i}^{2} \mathcal{T}+\left[H^{i}, H^{i}\right]_{\mathcal{T}}^{c}+\sum_{0 \leq s \leq \mathcal{T}}\left(\Delta H_{s}^{i}\right)^{2}\right)\right] & =\mathbb{E}\left[\left(-\sigma_{Z}^{2} \mathcal{T}+[Z, Z]_{\mathcal{T}}^{c}+\sum_{0 \leq s \leq \mathcal{T}}\left(\Delta Z_{s}\right)^{2}\right)\right] \\
& =\mathbb{E}\left[\left(-\rho^{i} \mathcal{T}+\int_{0+}^{\mathcal{T}} d\left[Z, H^{i}\right]_{s}^{c}+\sum_{0 \leq s \leq \mathcal{T}}\left(\Delta Z_{s} \Delta H_{s}^{i}\right)\right)\right] \\
& =0 .
\end{aligned}
$$

For the case of an exponentially distributed $\mathcal{T}$ that is independent of $\left\{Z, H^{i}\right\}$, we may apply law of iterated expectations (conditioning on $\mathcal{T}$ ) to show that these expectations are still zero.

Now, we let $\mathcal{G}_{\infty}^{i}$ be the $\sigma$-algebra generated by the path of $\left\{H_{t}^{i}, Z_{t}\right\}_{t=0}^{\infty}$, which is independent 
of $\mathcal{T}$ by assumption. Then

$$
\begin{aligned}
\mathbb{E}\left[\int_{0}^{\mathcal{T}}\left[v z_{s-}^{i}-V\left(z_{s-}^{i}, Z_{s-}^{i}\right)\right]\right. & \left.\left(d Y_{s}-r d s\right)\right] \\
= & \mathbb{E}\left[\mathbb{E}\left[\int_{0}^{\mathcal{T}}\left[v z_{s-}^{i}-V\left(z_{s-}^{i}, Z_{s-}^{i}\right)\right]\left(d Y_{s}-r d s\right) \mid \mathcal{G}_{\infty}^{i}\right]\right] \\
= & \mathbb{E}\left[\mathbb{E}\left[-r \int_{0}^{\mathcal{T}}\left[v z_{s-}^{i}-V\left(z_{s-}^{i}, Z_{s-}^{i}\right)\right] d s+v z_{\mathcal{T}}^{i}-V\left(z_{\mathcal{T}}^{i}, Z_{\mathcal{T}}^{i}\right) \mid \mathcal{G}_{\infty}^{i}\right]\right] \\
= & \mathbb{E}\left[-r \int_{0}^{\infty} r e^{-r t}\left(\int_{0}^{t}\left[v z_{s-}^{i}-V\left(z_{s-}^{i}, Z_{s-}^{i}\right)\right] d s\right) d t\right] \\
= & \mathbb{E}\left[-r \int_{0}^{\infty}\left(v z_{s-}^{i}-V\left(z_{s-}^{i}, Z_{s-}^{i}\right)\right) \int_{s}^{\infty} r e^{-r t} d t d s\right] \\
& +\mathbb{E}\left[\int_{0}^{\infty} r e^{-r t}\left(v z_{t}^{i}-V\left(z_{t}^{i}, Z_{t}^{i}\right)\right) d t\right] \\
& \mathbb{E}\left[-\int_{0}^{\infty}\left(v z_{s-}^{i}-V\left(z_{s-}^{i}, Z_{s-}^{i}\right)\right) r e^{-r s} d s\right] \\
+ & \mathbb{E}\left[\int_{0}^{\infty} r e^{-r t}\left(v z_{t}^{i}-V\left(z_{t}^{i}, Z_{t}^{i}\right)\right) d t\right]=0
\end{aligned}
$$

where the fourth equality follows from a change of order of integration from $\int_{0}^{\infty} \int_{0}^{t} d s d t$ to $\int_{0}^{\infty} \int_{s}^{\infty} d t d s$. Finally, we have already shown that $\mathbb{E}\left[\left(z_{s}^{i}\right)^{2}\right], \mathbb{E}\left[\left(z_{s}^{i}\right)\right], \mathbb{E}\left[\left(\bar{Z}_{s}\right)^{2}\right]$, and $\mathbb{E}\left[\left(\bar{Z}_{s}\right)\right]$ are all integrable. It then follows from Hölder's inequality that $\mathbb{E}\left[z_{s}^{i} \bar{Z}_{s}\right]$ is also integrable. Then $\left(\alpha_{1}+\alpha_{5} \bar{Z}_{s}+2 \alpha_{3} z_{s}^{i}\right)$ and $\left(\alpha_{2}+\alpha_{5} z_{s}^{i}+2 \alpha_{4} \bar{Z}_{s}\right)$ are square-integrable processes. So, for any $t$,

$$
\mathbb{E}\left[\int_{0+}^{t}\left(\alpha_{1}+\alpha_{5} \bar{Z}_{s-}+2 \alpha_{3} z_{s-}^{i}\right) d H_{s}^{i}\right]=\mathbb{E}\left[\int_{0+}^{t} \frac{1}{n}\left(\alpha_{2}+\alpha_{5} z_{s-}^{i}+2 \alpha_{4} \bar{Z}_{s-}\right) d Z_{s}\right]=0,
$$

since $H^{i}$ and $Z$ are martingales. The same result holds after replacing $t$ by $\mathcal{T}$, by applying the law of iterated expectations after conditioning on the independent exponentially distributed time $\mathcal{T}$. We have thus shown that taking an expectation in equation (49) gives

$$
\mathbb{E}\left[U\left(X_{\mathcal{T}}\right)-U\left(X_{0}\right)\right]=\mathbb{E}\left(\int_{0+}^{\mathcal{T}} \chi_{s} d s\right)
$$

Because $\alpha_{0}$ through $\alpha_{5}$ satisfy the system of equations specified at the beginning of this proof, we have

$$
\mathbb{E}\left[U\left(X_{\mathcal{T}}\right)-U\left(X_{0}\right)\right]=\mathbb{E}\left(\int_{0+}^{\mathcal{T}} \bar{\chi}_{s} d s\right)
$$


where

$$
\bar{\chi}_{s}=c\left(z_{s}^{i}-\bar{Z}_{s}\right) \frac{a+c \bar{Z}_{s}}{-b}+\gamma\left(z_{s}^{i}\right)^{2}-\lambda\left(R_{0}+R_{1} Z_{s}+R_{2} Z_{s}^{2}+R_{3} Z_{s} z_{s}^{i}+R_{4} z_{s}^{i}\right)
$$

Using the definitions of $U, \mathcal{T}$, and $R_{0}$ through $R_{4}$, as well as the fact that $\mathbb{E}\left[v z_{\mathcal{T}}^{i}\right]=\mathbb{E}\left[\pi z_{\mathcal{T}}^{i}\right]$, we can rearrange to find that

$$
\begin{aligned}
V\left(z_{0}^{i}, Z_{0}\right) & =\mathbb{E}\left[\pi z_{\mathcal{T}}^{i}+\int_{0+}^{\mathcal{T}} \bar{\chi}_{s} d s\right] \\
& =\mathbb{E}\left[\pi z_{\mathcal{T}}^{i}+\int_{0+}^{\mathcal{T}}-c\left(z_{s}^{i}-\bar{Z}_{s}\right) \frac{a+c \bar{Z}_{s}}{-b}-\gamma\left(z_{s}^{i}\right)^{2}+\lambda T^{R}\left(z_{s}^{i}, Z_{s}\right) d s\right] \\
& =\mathbb{E}\left[\pi z_{\mathcal{T}}^{i}+\int_{0}^{\mathcal{T}}-c\left(z_{s}^{i}-\bar{Z}_{s}\right) \frac{a+c \bar{Z}_{s}}{-b}-\gamma\left(z_{s}^{i}\right)^{2} d s+\int_{0}^{\mathcal{T}} T^{R}\left(z_{s}^{i}, Z_{s}\right) d N_{s}\right],
\end{aligned}
$$

which completes the proof.

\section{B.2 HJB conditions for optimality and individual rationality}

Here, we state the Hamilton-Jacobi-Bellman (HJB) equation, as a conjectured necessary condition on the candidate value function $V^{i}$ associated with the stochastic control problem of trader $i$. This conjectured condition is simply an aid to our later verification proof of the optimality of the candidate optimal demand policy, of the individual rationality condition for participation in size-discovery sessions, and of truthful inventory reporting in those sessions. We do not need to show that the HJB condition is appropriate or necessary for optimality, although both of these are in fact verified in the final verification step, which follows later in this sub-section. We also do not claim, yet, that the function $V^{i}$ specified by (9) is actually the continuation value function of trader $i$, although this also will also be shown later in the verification step.

For the purposes of this proof, we suppose that trader $i$ can observe the aggregate inventory $Z_{t}$. We show the corresponding optimal strategy depends only on the information actually available to trader $i$ (which does not include $Z_{t}$ ). The resulting strategy is therefore optimal even when restricted to the information actually available to trader $i$.

First, at any given state $\left(z^{i}, Z\right) \in \mathbb{R}^{2}$, consider the optimization problem faced by trader $i$ if a size-discovery session has just been declared. At that point, we suppose that the exchange price has already been fixed at some arbitrary level $p$, which for now is left as a free variable. Given the candidate continuation value function $V^{i}$, the candidate value for entering the size-discovery session is

$$
\mathcal{V}^{i}\left(z^{i}, Z, p\right) \equiv \sup _{\mu \in \mathcal{M}} V\left(z^{i}+Y^{i}\left(\left(\mu, z^{-i}\right)\right), Z\right)+T_{Q}^{i}\left(\left(\mu, z^{-i}\right), p\right) .
$$

The HJB equation for optimal exchange demand $d \in \mathbb{R}$ at any state $\left(z^{i}, Z\right)$ is

$$
0=r\left(z^{i} v-V\left(z^{i}, Z\right)\right)-\gamma\left(z^{i}\right)^{2}+\frac{\sigma_{i}^{2}}{2} V_{z z}\left(z^{i}, Z\right)+\frac{\sigma_{Z}^{2}}{2} V_{Z Z}\left(z^{i}, Z\right)+\rho^{i} V_{z Z}\left(z^{i}, Z\right)+\sup _{d} G(d),
$$


where

$$
G(d)=-\Phi_{(a, b, c)}\left(d ; Z-z^{i}\right) d+V_{z}^{i}\left(z^{i}, Z\right) d+\lambda\left(\mathcal{V}^{i}\left(z^{i}, Z, \Phi_{(a, b, c)}\left(d, Z-z^{i}\right)\right)-V^{i}\left(z^{i}, Z\right)\right) .
$$

The HJB equation reflects the fact that exchange demands are submitted before observing whether a size-discovery session will immediately follow, and reflect the influence of the demand $d$ on the exchange price $\Phi_{(a, b, c)}\left(d, Z-z^{i}\right)$ that would be used in size discovery, if it did immediately follow. Size-discovery sessions arrive at mean rate $\lambda$. Final asset payoffs arrive at mean rate $r$.

Proposition 5. Fix $\kappa_{0}<0$. For any demand coefficients $(a, b, c)$ with $2 c<r+\lambda$, let $V^{(a, b, c)}$ denote the candidate equilibrium value function defined by equation (47) in Lemma 3, for the $R_{0}-R_{4}$ that correspond to $\kappa_{0}, a, b, c$. The candidate value function $V^{(a, b, c)}$ solves the HJB equation (51) if and only if the following conditions hold:

1. The coefficient $b$ is real and given by one of the following two values:

$$
b=\frac{r^{2}}{8 \gamma}\left(-(n-2)+\frac{3 \lambda}{r} \pm \sqrt{\left(-(n-2)+\frac{3 \lambda}{r}\right)^{2}-\frac{8 \lambda(r+\lambda)}{r^{2}}}\right)
$$

2. The coefficients a, $c$ are given by equations $(14,16)$.

If these conditions hold, the candidate value function $V^{i}: \mathbb{R}^{2} \rightarrow \mathbb{R}$ specified by (9) solves the HJB equation (51). At any given state $\left(z^{i}, Z\right)$, and at the price $p=P(\bar{Z})$, the solution to the associated size-discovery problem (50) is $\mu^{i}=z^{i}$, that is, to participate and to report truthfully. The supremum for the exchange demand optimization problem (51) is attained at $a+b P(\bar{Z})+c z^{i}$, consistent with the given demand function coefficients $(a, b, c)$.

Finally, there exist two real negative solutions to equation (52) if $\lambda \leq \bar{\lambda}$, and the corresponding c coefficients given by ((16)) satisfy $2 c<r+\lambda$. There are no real solutions to (52) if $\lambda>\bar{\lambda}$.

A proof of this proposition is left to subsection B.3. Exploiting Proposition 5, we will then use a standard verification argument in subsection B.4 to show that, under the conditions of Proposition $5, V^{i}\left(z_{0}^{i}, Z_{0}\right)$ is equal to the value of the candidate equilibrium strategy $\left(D^{i}, \mu^{i}\right)$, and dominates the value of any other strategy, completing the proof of equilibrium.

\section{B.3 Proof of Proposition 5}

We turn to a proof of Proposition 5. This involves a lengthy calculation of the coefficients $(a, b, c)$ of the demand function and the coefficients $(\alpha, R)$ of the linear-quadratic candidate form of the value function $V^{i}$ satisfying the properties stated by Proposition 5 .

Based on the given form (47) of the candidate value function $V^{i}$, we note that for any real number $y, V^{i}\left(z^{i}+y, Z\right)$ differs by only a constant from

$$
\left(\alpha_{1}+\alpha_{5} \bar{Z}\right) y+\alpha_{3} y^{2}+2 \alpha_{3} z^{i} y
$$


In the HJB equation (51), from the fact that the total of the other traders' candidate equilibrium reports is $\sum_{j \neq i} z^{j}=Z-z^{i}$, trader $i$ gets a cash transfer at given demand $d \in \mathbb{R}$ of

$$
\kappa_{0}\left(-n \beta\left(p_{d}\right)+Z-z^{i}+\mu^{i}\right)^{2}+p_{d}\left(\mu^{i}-\beta\left(p_{d}\right)\right)+\frac{p_{d}^{2}}{4 \kappa_{0} n^{2}},
$$

where $p_{d}=\Phi_{(a, b, c)}\left(d ; Z-z^{i}\right)$, and an asset transfer given a report $\mu^{i}$ of

$$
Y^{i}\left(\left(\mu^{i}, z^{-i}\right)\right)=\frac{Z-z^{i}}{n}-\frac{n-1}{n} \mu^{i} .
$$

Assuming temporarily the IR condition that $\mu^{i} \neq \nu$, the optimization problem faced by trader $i$ is equivalent to maximizing the sum of (i) the quantity $-\Phi_{(a, b, c)}\left(d ; Z-z^{i}\right) d+V_{z}\left(z^{i}, Z\right) d$ and (ii) the product of $\lambda$ with

$$
\begin{aligned}
\mathcal{E}\left(p_{d}, Z, z^{i}, \mu^{i}\right) & \equiv\left(\alpha_{1}+\alpha_{5} \bar{Z}\right)\left(\frac{Z-z^{i}}{n}-\frac{n-1}{n} \mu^{i}\right)+\alpha_{3}\left(\frac{Z-z^{i}}{n}-\frac{n-1}{n} \mu^{i}\right)^{2} \\
& +2 \alpha_{3} z^{i}\left(\frac{Z-z^{i}}{n}-\frac{n-1}{n} \mu^{i}\right)+\kappa_{0}\left(-n \beta\left(p_{d}\right)+Z-z^{i}+\mu^{i}\right)^{2} \\
& +p_{d}\left(\mu^{i}-\beta\left(p_{d}\right)\right)+\frac{p_{d}^{2}}{4 \kappa_{0} n^{2}} .
\end{aligned}
$$

The first-order condition for optimality of $\mu^{i}$ is

$$
\begin{aligned}
\frac{\partial \mathcal{E}\left(p_{d}, Z, z^{i}, \mu^{i}\right)}{\partial \mu^{i}}=- & \frac{n-1}{n}\left(\alpha_{1}+\alpha_{5} \bar{Z}\right)+\frac{2(n-1)^{2}}{n^{2}} \alpha_{3} \mu^{i}-2 \frac{n-1}{n} \alpha_{3} \frac{Z-z^{i}}{n} \\
& -\frac{n-1}{n} 2 \alpha_{3} z^{i}+2 \kappa_{0}\left(-n \beta\left(p_{d}\right)+\mu^{i}+Z-z^{i}\right)+p_{d}=0 .
\end{aligned}
$$

The second-order condition is satisfied if $\alpha_{3}<0$ and $\kappa_{0}<0$. For the candidate equilibrium strategy $\mu^{i}=z^{i}$, we have

$$
\frac{\partial \mathcal{E}\left(p_{d}, Z, z^{i}, \mu^{i}\right)}{\partial \mu^{i}}=-\frac{n-1}{n}\left(\alpha_{1}+\alpha_{5} \bar{Z}\right)+\frac{2(n-1) \alpha_{3}}{n}(-\bar{Z})+2 \kappa_{0}\left(-n \beta\left(p_{d}\right)+Z\right)+p_{d} .
$$

For notational simplicity, from this point we write simply $p$ for the price $p_{d}$ at the given demand $d$. Plugging in

$$
Z=n \frac{-b p-a}{c},
$$

which must hold in a symmetric equilibrium, and writing $\beta(p)=-\hat{a}-\hat{b} p$, we have

$$
\begin{aligned}
\frac{\partial \mathcal{E}\left(p, Z, z^{i}, \mu^{i}\right)}{\partial \mu^{i}}=- & \frac{n-1}{n}\left(\alpha_{1}+\alpha_{5} \frac{-b p-a}{c}\right)+\frac{2(n-1) \alpha_{3}}{n} \frac{b p+a}{c} \\
& +2 \kappa_{0}\left(n \hat{a}+n \hat{b} p+n \frac{-b p-a}{c}\right)+p .
\end{aligned}
$$


The candidate equilibrium strategy $\mu^{i}=z^{i}$ is therefore optimal provided that

$$
\begin{aligned}
& 0=-\frac{n-1}{n}\left(\alpha_{1}-\frac{\alpha_{5} a}{c}\right)+\frac{2(n-1) \alpha_{3} a}{n c}+2 \kappa_{0} n \hat{a}-\frac{2 n a \kappa_{0}}{c} \\
& 0=\frac{n-1}{n}\left(\frac{\alpha_{5} b}{c}\right)+\frac{2(n-1) \alpha_{3} b}{n c}+2 \kappa_{0} n\left(\hat{b}-\frac{b}{c}\right)+1,
\end{aligned}
$$

or equivalently,

$$
\begin{aligned}
& \hat{a}=\frac{a}{c}-\frac{1}{2 n \kappa_{0}}\left(-\frac{n-1}{n}\left(\alpha_{1}-\frac{\alpha_{5} a}{c}\right)+\frac{2(n-1) \alpha_{3} a}{n c}\right) \\
& \hat{b}=\frac{b}{c}-\frac{1}{2 n \kappa_{0}}\left(\frac{n-1}{n}\left(\frac{\alpha_{5} b}{c}\right)+\frac{2(n-1) \alpha_{3} b}{n c}+1\right) .
\end{aligned}
$$

These equations imply that

$$
\begin{aligned}
\zeta \equiv n \hat{a}+n \hat{b} \frac{a+c \bar{Z}}{-b}+Z \\
=-\frac{1}{2 \kappa_{0}}\left(-\frac{n-1}{n}\left(\alpha_{1}-\frac{\alpha_{5} a}{c}\right)+\frac{2(n-1) \alpha_{3} a}{n c}\right) \\
\quad-\frac{1}{2 \kappa_{0}}\left(\frac{a+c \bar{Z}}{-b}\right)\left(\frac{n-1}{n}\left(\frac{\alpha_{5} b}{c}\right)+\frac{2(n-1) \alpha_{3} b}{n c}+1\right) .
\end{aligned}
$$

Evaluating this expression for $\zeta$ at $p=-(a+c \bar{Z}) / b$, we have

$$
\zeta=\frac{-1}{2 \kappa_{0}}\left(p-\frac{n-1}{n} \alpha_{1}+\frac{n-1}{n} \alpha_{5} \frac{a+b p}{c}+\frac{2(n-1) \alpha_{3}}{n} \frac{a+b p}{c}\right) .
$$

Now, consider the IR condition associated with the HJB equation (51), that the optimal choice for $\mu^{i}$ is an actual inventory report, as we have considered so far, rather than the nonparticipation choice $\nu$. From (51), this IR condition is that the sum of the cash transfer at the optimal inventory report and the change in utility, $V(\bar{Z}, Z)-V\left(z^{i}, Z\right)$, must be weakly positive. This must hold at all possible $z \in \mathbb{R}^{n}$, even if all traders have the average inventory $\bar{Z}$ when entering the size-discovery session. In particular, the sum of the cash transfers must be weakly positive in this case, but is always weakly negative by budget balance, so the cash transfers must sum to 0 . In general, the sum of the cash transfers is

$$
-n\left(\sqrt{-\kappa_{0}}\left(-n \beta(p)+\sum_{j=1}^{n} \mu^{j}\right)-\frac{p}{2 n \sqrt{-\kappa_{0}}}\right)^{2} .
$$


So, if the transfers are to sum to 0 , it must be that

$$
\sqrt{-\kappa_{0}}\left(-n \beta(p)+\sum_{j=1}^{n} \mu^{j}\right)-\frac{p}{2 n \sqrt{-\kappa_{0}}}=0
$$

and

$$
\left|\kappa_{0}\right|\left(-n \beta(p)+\sum_{j=1}^{n} \mu^{j}\right)-\frac{p}{2 n}=-\kappa_{0}\left(-n \beta(p)+\sum_{j=1}^{n} \mu^{j}\right)-\frac{p}{2 n}=0 .
$$

Recall from equation (55) that at the equilibrium strategies and the choice for $\beta(p)$ that is consistent with optimality, we have

$$
-n \beta(p)+\sum_{j=1}^{n} \mu^{j}=\frac{-1}{2 \kappa_{0}}\left(p-\frac{n-1}{n} \alpha_{1}+\frac{n-1}{n} \alpha_{5} \frac{a+b p}{c}+\frac{2(n-1) \alpha_{3}}{n} \frac{a+b p}{c}\right) .
$$

Thus, for the IR condition $\mu^{i} \neq \nu$ to hold, combined with (56), it must be that

$$
\begin{aligned}
& \frac{1}{2}\left(\frac{n-1}{n} p-\frac{n-1}{n} \alpha_{1}+\frac{n-1}{n} \alpha_{5} \frac{a+b p}{c}+\frac{2(n-1) \alpha_{3}}{n} \frac{a+b p}{c}\right) \\
& \quad=\frac{1}{2}\left(\frac{n-1}{n} p-\frac{n-1}{n} \alpha_{1}-\frac{n-1}{n} \alpha_{5} \bar{Z}-\frac{2(n-1) \alpha_{3}}{n} \bar{Z}\right) \\
& \quad=0 .
\end{aligned}
$$

Put differently, for the equilibrium strategies to satisfy the IR condition, we need the condition

$$
p=\alpha_{1}+\left(\alpha_{5}+2 \alpha_{3}\right) \bar{Z}
$$

We conjecture and later verify that (57) holds in equilibrium. Given this, we see that, in equilibrium,

$$
-n \beta(p)+\sum_{j=1}^{n} \mu^{j}=\frac{-p}{2 \kappa_{0} n}
$$

Likewise, we see that

$$
\begin{aligned}
& -\beta(p)+\mu^{i}=\hat{a}+\hat{b} \frac{a+c \bar{Z}}{-b}+z^{i} \\
& \quad=z^{i}-\bar{Z}-\frac{1}{2 \kappa_{0} n}\left(p-\frac{n-1}{n} \alpha_{1}+\frac{n-1}{n} \alpha_{5} \frac{a+b p}{c}+\frac{2(n-1) \alpha_{3}}{n} \frac{a+b p}{c}\right) \\
& \quad=z^{i}-\bar{Z}-\frac{p}{2 \kappa_{0} n^{2}} .
\end{aligned}
$$


Now, if we plug $\beta(p)=-\hat{a}-\hat{b} p$ into the definition of $\mathcal{E}\left(p, Z, z^{i}, \mu^{i}\right)$, we arrive at

$$
\begin{aligned}
\mathcal{E}\left(p, Z, z^{i}, \mu^{i}\right)=( & \left.\alpha_{1}+\alpha_{5} \bar{Z}\right)\left(\frac{Z-z^{i}}{n}-\frac{n-1}{n} \mu^{i}\right) \\
& +\alpha_{3}\left(\frac{Z-z^{i}}{n}-\frac{n-1}{n} \mu^{i}\right)^{2}+2 \alpha_{3} z^{i}\left(\frac{Z-z^{i}}{n}-\frac{n-1}{n} \mu^{i}\right) \\
& +\kappa_{0}\left(n(\hat{a}+\hat{b} p)+Z-z^{i}+\mu^{i}\right)^{2}+p\left(\mu^{i}+(\hat{a}+\hat{b} p)\right)+\frac{p^{2}}{4 \kappa_{0} n^{2}} .
\end{aligned}
$$

The partial derivative of $\mathcal{E}\left(p, Z, z^{i}, \mu^{i}\right)$ with respect to $p$ is then

$$
\mathcal{E}_{p}\left(p, Z, z^{i}, \mu^{i}\right)=2 \kappa_{0} n \hat{b}\left(n(\hat{a}+\hat{b} p)+Z-z^{i}+\mu^{i}\right)+\left(\mu^{i}+(\hat{a}+2 \hat{b} p)\right)+\frac{p}{2 \kappa_{0} n^{2}} .
$$

Plugging in the candidate $\mu^{i}=z^{i}$ and the fact from above that $\hat{a}+\hat{b} p=-\bar{Z}-p /\left(2 \kappa_{0} n^{2}\right)$, we have

$$
\mathcal{E}_{p}\left(p, Z, z^{i}, \mu^{i}\right)=2 \kappa_{0} n \hat{b} \frac{-p}{2 \kappa_{0} n}+\hat{b} p+\left(z^{i}-\bar{Z}-\frac{p}{2 \kappa_{0} n^{2}}\right)+\frac{p}{2 \kappa_{0} n^{2}}=z^{i}-\bar{Z} .
$$

Finally, for the candidate equilibrium reports and exchange demands, the associated cash transfers are

$$
\begin{aligned}
\kappa_{0}\left(-n \beta(p)+\sum_{j=1}^{n} \mu^{j}\right)^{2}+p\left(\mu^{i}-\beta(p)\right)+\frac{p^{2}}{4 \kappa_{0} n^{2}} & =\frac{p^{2}}{4 \kappa_{0} n^{2}}+p\left(z^{i}-\bar{Z}-\frac{p}{2 \kappa_{0} n^{2}}\right)+\frac{p^{2}}{4 \kappa_{0} n^{2}} \\
& =p\left(z^{i}-\bar{Z}\right) \\
& =\frac{a+c \bar{Z}}{-b}\left(z^{i}-\bar{Z}\right)
\end{aligned}
$$

which implies that

$$
\begin{aligned}
R_{0} & =0 \\
R_{1} & =\frac{a}{n b} \\
R_{2} & =\frac{c}{n^{2} b} \\
R_{3} & =\frac{c}{-n b} \\
R_{4} & =\frac{a}{-b} .
\end{aligned}
$$

The HJB optimization problem (51) to solve is equivalent to

$$
\sup _{d, \mu^{i}}-\Phi_{(a, b, c)}\left(d ; Z-z^{i}\right) d+V_{z}\left(z^{i}, Z\right) d+\lambda \mathcal{E}\left(\Phi_{(a, b, c)}\left(d ; Z-z^{i}\right), Z, z^{i}, \mu^{i}\right) .
$$


Taking derivatives with respect to $d$ and $\mu^{i}$, respectively, we need the first-order conditions

$$
\begin{aligned}
0= & -\Phi_{(a, b, c)}\left(d ; Z-z^{i}\right)-d \frac{\partial \Phi_{(a, b, c)}\left(d ; Z-z^{i}\right)}{\partial d}+V_{z}\left(z^{i}, Z\right) \\
& +\lambda \frac{\partial \Phi_{(a, b, c)}\left(d ; Z-z^{i}\right)}{\partial d} \mathcal{E}_{p}\left(\Phi_{(a, b, c)}\left(d ; Z-z^{i}\right), Z, z^{i}, \mu^{i}\right)
\end{aligned}
$$

and

$$
0=\mathcal{E}_{\mu^{i}}\left(\Phi_{(a, b, c)}\left(d ; Z-z^{i}\right), Z, z^{i}, \mu^{i}\right)
$$

We conjecture and later verify that the solution to these first-order conditions is a maximum. To prove the result, these equalities must hold at $d=a+b p+c z^{i}$, implying that

$$
\Phi_{(a, b, c)}\left(d ; Z-z^{i}\right)=\frac{a+c \bar{Z}}{-b}
$$

and at $\mu^{i}=z^{i}$. We recall that

$$
\frac{\partial \Phi_{(a, b, c)}\left(d ; Z-z^{i}\right)}{\partial d}=\frac{-1}{b(n-1)}
$$

From the above, the second equation is satisfied at $p=-(a+c \bar{Z}) / b$ and at the conjectured $\mu^{i}$ as long as

$$
\begin{aligned}
& 0=-\frac{n-1}{n}\left(\alpha_{1}-\frac{\alpha_{5} a}{c}\right)+\frac{2(n-1) \alpha_{3} a}{n c}+2 \kappa_{0} n \hat{a}-\frac{2 n a \kappa_{0}}{c} \\
& 0=\frac{n-1}{n}\left(\frac{\alpha_{5} b}{c}\right)+\frac{2(n-1) \alpha_{3} b}{n c}+2 \kappa_{0} n\left(\hat{b}-\frac{b}{c}\right)+1
\end{aligned}
$$

where we have written $\beta(p)$ as $\beta(p)=-\hat{a}-\hat{b} p$. For the first-order condition on the demand $d$, we need

$$
-p+\frac{1}{b(n-1)}\left(a+b p+c z^{i}\right)+\left(\alpha_{1}+2 \alpha_{3} z^{i}+\alpha_{5} \bar{Z}\right)-\frac{\lambda}{b(n-1)} \mathcal{E}_{p}\left(p, Z, z^{i}, \mu^{i}\right)=0 .
$$

We showed that, at equilibrium, $\mathcal{E}_{p}=z^{i}-\bar{Z}$. Plugging this in, and using $\bar{Z}=(-b p-a) / c$, we see that

$$
-p+\frac{1}{b(n-1)}\left(a+b p+c z^{i}\right)+\left(\alpha_{1}+2 \alpha_{3} z^{i}+\alpha_{5} \frac{-b p-a}{c}\right)-\frac{\lambda}{b(n-1)}\left(z^{i}-\frac{-b p-a}{c}\right)=0 .
$$


Gathering terms,

$$
\begin{aligned}
& 0=-1+\frac{1}{(n-1)}-\alpha_{5} \frac{b}{c}-\frac{\lambda}{c(n-1)} \\
& 0=\frac{1}{b(n-1)} c+2 \alpha_{3}-\frac{\lambda}{b(n-1)} \\
& 0=\frac{1}{b(n-1)} a+\left(\alpha_{1}+\alpha_{5} \frac{-a}{c}\right)-\frac{\lambda}{b(n-1)} \frac{a}{c} .
\end{aligned}
$$

Rearranging,

$$
\begin{aligned}
& 0=-(n-2) c-\alpha_{5}(n-1) b-\lambda \\
& c=-2 \alpha_{3} b(n-1)+\lambda,
\end{aligned}
$$

while from the derivation of the linear-quadratic value function, we have

$$
\begin{aligned}
\alpha_{3} & =\frac{-\gamma}{r+\lambda-2 c} \\
\alpha_{5} & =\frac{1}{r+\lambda-c}\left(\frac{c^{2}}{b}-2 \alpha_{3} c+n \lambda R_{3}\right),
\end{aligned}
$$

where $R_{3}$ is the coefficient on $Z z$ in the cash transfer. From the last section, in equilibrium we have $R_{3}=c /(-n b)$ and thus the relevant system of equations is

$$
\begin{aligned}
\alpha_{3} & =\frac{-\gamma}{r+\lambda-2 c} \\
\alpha_{5} & =\frac{1}{r+\lambda-c}\left(\frac{c^{2}}{b}-2 \alpha_{3} c-\frac{\lambda c}{b}\right) .
\end{aligned}
$$

Multiplying both sides of the equation for $\alpha_{5}$ by $b(n-1)$, we have

$$
\alpha_{5} b(n-1)=\frac{1}{r+\lambda-c}\left(c^{2}(n-1)-2 \alpha_{3} b(n-1) c-\lambda c(n-1)\right),
$$

and plugging in the above, we have

$$
\alpha_{5} b(n-1)=\frac{n c}{r+\lambda-c}(c-\lambda),
$$




$$
\begin{aligned}
& 0=-(n-2) c-\left(\frac{n c}{r+\lambda-c}(c-\lambda)\right)-\lambda \\
& 0=-(n-2) c(r+\lambda-c)-n c(c-\lambda)-\lambda(r+\lambda-c) \\
& 0=-2 c^{2}+c(-(n-2)(r+\lambda)+n \lambda+\lambda)-\lambda(r+\lambda) \\
& 0=-2 c^{2}+c(-(n-2) r+3 \lambda)-\lambda(r+\lambda) \\
& c=\frac{(-(n-2) r+3 \lambda) \pm \sqrt{(-(n-2) r+3 \lambda)^{2}-8 \lambda(r+\lambda)}}{4} .
\end{aligned}
$$

It is clear that either both of the roots or neither of the roots are real. By the Déscartes rule of signs, if both are real, they are either both positive, or neither are positive. In particular, assuming that $(-(n-2) r+3 \lambda)^{2}-8 \lambda(r+\lambda)>0$ so that both roots exist, if we can show one is negative then they both are negative. If $-(n-2) r+3 \lambda<0$, then the smaller root must be negative and we are done. If $-(n-2) r+3 \lambda \geq 0$, then the larger root is positive so both roots are positive. Thus we see we need that $-(n-2) r+3 \lambda<0$ and $(-(n-2) r+3 \lambda)^{2}-8 \lambda(r+\lambda) \geq 0$, which can be concisely written as

$$
-(n-2) r+3 \lambda \leq-\sqrt{8 \lambda(r+\lambda)}
$$

Define

$$
F(c, \lambda)=-2 c^{2}+c(-(n-2) r+3 \lambda)-\lambda(r+\lambda) .
$$

For each fixed $\lambda$, an equilibrium is determined by any $c<0$ satisfying $F(c, \lambda)=0$. The condition that $c<0$ is equivalent to $b<0$, which ensures that the second-order condition above holds.

We have that $F_{c c}=-4<0$ and $\lim _{c \rightarrow-\infty} F=\lim _{c \rightarrow \infty} F=-\infty$. Thus, as $c$ increases from negative infinity to infinity, $F_{c}$ crosses from positive to negative exactly once, at

$$
c_{0}=\frac{-(n-2) r+3 \lambda}{4} \text {. }
$$

Since there are two roots, we see the derivative $F_{c}$ must be positive at the smaller root $\underline{c}(\lambda)$ and negative at the larger root $\bar{c}(\lambda)$, so $\underline{c}(\lambda)<c_{0}<\bar{c}(\lambda)$. Fix a $\lambda \in(0, \bar{\lambda})$ and consider small, disjoint neighborhoods around $(\lambda, \bar{c}(\lambda))$ and $(\lambda, \underline{c}(\lambda))$. Applying the implicit function theorem to each of these functions,

$$
\frac{\partial c}{\partial \lambda}=-\frac{F_{\lambda}}{F_{c}}=-\frac{-r-2 \lambda+3 c}{F_{c}}
$$

Since $c<0$ in either equilibrium, the numerator is always negative. We just showed that $F_{c}$ is positive at the smaller root and thus that $\frac{\partial \underline{c}(\lambda)}{\partial \lambda}>0$, so that $c$ increases monotonically in $\lambda$. Also, since $c<0$, it is clear that $2 c<r+\lambda$ so the corresponding demand process is admissible.

Now, recall that

$$
(r+\lambda-2 c) \alpha_{3}=-\gamma
$$


which, combined with equation (63), implies that

$$
\begin{aligned}
& c(r+\lambda-2 c)=-2 \alpha_{3} b(n-1)(r+\lambda-2 c)+\lambda(r+\lambda-2 c) \\
& c(r+\lambda-2 c)=2 \gamma b(n-1)+\lambda(r+\lambda-2 c) .
\end{aligned}
$$

Using the above quadratic equation for $c$, this can be rewritten

$$
\begin{aligned}
c(r+\lambda)-(c(-(n-2) r+3 \lambda)-\lambda(r+\lambda)) & =2 \gamma b(n-1)+\lambda(r+\lambda-2 c) \\
c(r+\lambda)-(c(-(n-2) r+3 \lambda)) & =2 \gamma b(n-1)-2 \lambda c \\
c r(n-1) & =2 \gamma b(n-1) \\
c & =\frac{2 \gamma}{r} b,
\end{aligned}
$$

which implies that

$$
b=\frac{r^{2}}{8 \gamma}\left(-(n-2)+\frac{3 \lambda}{r} \pm \sqrt{\left(-(n-2)+\frac{3 \lambda}{r}\right)^{2}-\frac{8 \lambda(r+\lambda)}{r^{2}}}\right)
$$

We note that

$$
\begin{aligned}
{\left[\frac{3 \lambda}{r}-(n-2)\right]^{2}-\frac{8 \lambda(r+\lambda)}{r^{2}} } & =\frac{\lambda^{2}}{r^{2}}-\frac{6 \lambda(n-2)}{r}+(n-2)^{2}-\frac{8 \lambda}{r} \\
& =\left(\frac{\lambda}{r}-(n-2)\right)^{2}-\frac{4 \lambda n}{r}
\end{aligned}
$$

Thus, we have shown that

$$
b=\frac{-r^{2}}{8 \gamma}\left((n-2)-\frac{3 \lambda}{r} \pm \sqrt{\left(\frac{\lambda}{r}-(n-2)\right)^{2}-\frac{4 \lambda n}{r}}\right) .
$$

Further, since $c<0$ and $c=2 \gamma b / r$, we have $b<0$, and since $c$ increases monotonically in $\lambda$ so does $b$. Using the relation that $c=2 \gamma b / r$ and equation (63), we have

$$
\alpha_{3}=\frac{c-\lambda}{-2 b(n-1)}=-\frac{\gamma}{r(n-1)}+\frac{\lambda}{2 b(n-1)} \text {. }
$$


Using (62), we now have

$$
\begin{aligned}
0 & =-(n-2) c-\alpha_{5}(n-1) b-\lambda \\
\alpha_{5} & =\frac{-(n-2) c-\lambda}{b(n-1)} \\
& =-\frac{n-2}{n-1} \frac{2 \gamma}{r}-\frac{\lambda}{b(n-1)} \\
& =\frac{-2 \gamma}{r}-2 \alpha_{3} .
\end{aligned}
$$

Recall that

$$
\alpha_{1}=\frac{1}{r+\lambda-c}\left(r v+\frac{a c}{b}+\lambda R_{4}\right),
$$

where, based on the transfers, $R_{4}=-a / b$, so

$$
\alpha_{1}=\frac{1}{r+\lambda-c}\left(r v+\frac{a c}{b}-\frac{a \lambda}{b}\right) .
$$

From the first-order condition for auction demand,

$$
0=\frac{1}{b(n-1)} a+\left(\alpha_{1}+\alpha_{5} \frac{-a}{c}\right)-\frac{\lambda}{b(n-1)} \frac{a}{c} .
$$

Plugging in

$$
\alpha_{5}=\frac{-2 \gamma}{r}-2\left(\frac{c-\lambda}{-2 b(n-1)}\right)
$$

we have

$$
0=\alpha_{1}+\frac{2 \gamma}{r} \frac{a}{c}
$$

implying that

$$
\alpha_{1}=-\frac{a}{b} .
$$

Now, plugging this into the above, we have

$$
\alpha_{1}=\frac{1}{r+\lambda-c}\left(r v+-c \alpha_{1}+\lambda \alpha_{1}\right)
$$

from which it is clear that $\alpha_{1}=v$ and $a=-b v$. Returning to the coefficients $\hat{a}, \hat{b} \operatorname{defining} \beta(p)$, since

$$
\frac{a}{c}=-v \frac{r}{2 \gamma}
$$

and

$$
\frac{b}{c}=\frac{r}{2 \gamma}
$$


we have

$$
\begin{aligned}
\hat{a} & =\frac{a}{c}-\frac{1}{2 n \kappa_{0}}\left(-\frac{n-1}{n}\left(\alpha_{1}-\frac{\alpha_{5} a}{c}\right)+\frac{2(n-1) \alpha_{3} a}{n c}\right) \\
& =\frac{-v r}{2 \gamma}-\frac{1}{2 n \kappa_{0}}\left(-\frac{n-1}{n}\left(v-v\left(\frac{2 \gamma}{r}\right)\left(\frac{r}{2 \gamma}\right)\right)\right) \\
& =\frac{-v r}{2 \gamma} \\
\hat{b} & =\frac{b}{c}-\frac{1}{2 n \kappa_{0}}\left(\frac{n-1}{n}\left(\frac{\alpha_{5} b}{c}\right)+\frac{2(n-1) \alpha_{3} b}{n c}+1\right) \\
& =\frac{r}{2 \gamma}-\frac{1}{2 n^{2} \kappa_{0}} .
\end{aligned}
$$

Returning to the system of value function coefficients, it remains to calculate

$$
\begin{aligned}
\alpha_{4} & =\frac{1}{r}\left(\frac{c^{2}}{-b}+(\lambda-c) \alpha_{5}+\lambda \alpha_{3}+\lambda n^{2} R_{2}\right) \\
\alpha_{2} & =\frac{1}{r}\left(\frac{c a}{-b}+(\lambda-c) \alpha_{1}+\lambda n R_{1}\right) \\
\alpha_{0}^{i} & =\frac{1}{r}\left(\alpha_{3} \sigma_{i}^{2}+\alpha_{4} \frac{\sigma_{Z}^{2}}{n^{2}}+\alpha_{5} \frac{\rho^{i}}{n}+\lambda R_{0}\right) .
\end{aligned}
$$

Plugging in the equilibrium formulas for $R_{2}, R_{1}$, and $R_{0}$, we have

$$
\begin{aligned}
\alpha_{4} & =\frac{1}{r}\left(\frac{c^{2}}{-b}+(\lambda-c) \alpha_{5}+\lambda \alpha_{3}+\frac{c \lambda}{b}\right) \\
\alpha_{2} & =\frac{1}{r}\left(\frac{c a}{-b}+(\lambda-c) v+\frac{a \lambda}{b}\right) \\
\alpha_{0}^{i} & =\frac{1}{r}\left(\alpha_{3} \sigma_{i}^{2}+\alpha_{4} \frac{\sigma_{Z}^{2}}{n^{2}}+\alpha_{5} \frac{\rho^{i}}{n}\right) .
\end{aligned}
$$

Using the definitions of $a, b, c$, we thus have

$$
\begin{aligned}
\alpha_{4} & =\frac{1}{r}\left(-\frac{2 \gamma}{r} c+(\lambda-c)\left(\frac{-2 \gamma}{r}-2 \alpha_{3}\right)+\lambda \alpha_{3}+\frac{c \lambda}{b}\right) \\
\alpha_{2} & =\frac{1}{r}(c v+(\lambda-c) v+-v \lambda),
\end{aligned}
$$


implying that $\alpha_{2}=0$ and that

$$
\begin{aligned}
\alpha_{4} & =\frac{1}{r}\left(2 c \alpha_{3}+\lambda\left(\frac{-2 \gamma}{r}-2 \alpha_{3}\right)+\lambda \alpha_{3}+\frac{2 \gamma \lambda}{r}\right) \\
& =\frac{1}{r}(2 c-\lambda) \alpha_{3}=\frac{\gamma}{r}+\alpha_{3} .
\end{aligned}
$$

Finally, this implies that

$$
\begin{aligned}
\alpha_{0}^{i} & =\frac{1}{r}\left(\frac{\gamma}{r} \frac{\sigma_{Z}^{2}}{n^{2}}+\alpha_{3}\left(\frac{\sigma_{Z}^{2}}{n^{2}}+\sigma_{i}^{2}\right)+\alpha_{5} \frac{\rho^{i}}{n}\right) \\
& =\frac{1}{r}\left(\frac{\gamma}{r} \frac{\sigma_{Z}^{2}}{n^{2}}+\alpha_{3}\left(\frac{\sigma_{Z}^{2}}{n^{2}}+\sigma_{i}^{2}-2 \frac{\rho^{i}}{n}\right)-\frac{2 \gamma}{r} \frac{\rho^{i}}{n}\right) \\
& =\frac{1}{r}\left(\frac{\gamma}{r} \frac{\sigma_{Z}^{2}}{n^{2}}+\left(-\frac{\gamma}{r(n-1)}+\frac{\lambda}{2 b(n-1)}\right)\left(\frac{\sigma_{Z}^{2}}{n^{2}}+\sigma_{i}^{2}-2 \frac{\rho^{i}}{n}\right)-\frac{2 \gamma}{r} \frac{\rho^{i}}{n}\right) .
\end{aligned}
$$

Note that

$$
\frac{\sigma_{Z}^{2}}{n^{2}}+\sigma_{i}^{2}-2 \frac{\rho^{i}}{n}
$$

is the variance of $Z_{1} / n-H_{1}^{i}$ conditional on $Z_{0}$, and is thus positive, so $\alpha_{0}^{i}$ declines in $\lambda$ because $b<0$ and because $b$ increases with $\lambda$.

\section{B.3.1 Confirming conjectures}

All that remains is to check that the conjectured conditions are true. First, we must verify the equilibrium condition

$$
p=\alpha_{1}+\left(\alpha_{5}+2 \alpha_{3}\right) \bar{Z} .
$$

We see from the definitions of $a, b$, and $c$ that

$$
p=\frac{a+c \bar{Z}}{-b}=v-\frac{2 \gamma}{r} \bar{Z},
$$

as hypothesized, while from the definition of $\alpha_{5}, \alpha_{3}$ we have

$$
2 \alpha_{3}+\alpha_{5}=\frac{-2 \gamma}{r}
$$

so (68) indeed holds.

Next, we must confirm that the equilibrium exchange process and reporting process that satisfy the first-order conditions for the optimization (59) correspond to a maximum. Recall 
that

$$
\begin{aligned}
\mathcal{E}\left(\phi, Z, z^{i}, \mu^{i}\right) & \equiv\left(\alpha_{1}+\alpha_{5} \bar{Z}\right)\left(\frac{Z-z^{i}}{n}-\frac{n-1}{n} \mu^{i}\right)+\alpha_{3}\left(\frac{Z-z^{i}}{n}-\frac{n-1}{n} \mu^{i}\right)^{2} \\
& +2 \alpha_{3} z^{i}\left(\frac{Z-z^{i}}{n}-\frac{n-1}{n} \mu^{i}\right)+\kappa_{0}\left(-n \beta(\phi)+Z-z^{i}+\mu^{i}\right)^{2}+\phi\left(\mu^{i}-\beta(\phi)\right)+\frac{\phi^{2}}{4 \kappa_{0} n^{2}} .
\end{aligned}
$$

Direct calculation shows

$$
\begin{gathered}
\frac{\partial \mathcal{E}\left(\phi, Z, z^{i}, \mu^{i}\right)}{\partial \mu^{i}}=-\frac{n-1}{n}\left(\alpha_{1}+\alpha_{5} \bar{Z}\right)+\frac{2(n-1)^{2}}{n^{2}} \alpha_{3} \mu^{i}-2 \frac{n-1}{n} \alpha_{3} \frac{Z-z^{i}}{n} \\
-\frac{n-1}{n} 2 \alpha_{3} z^{i}+2 \kappa_{0}\left(-n \beta(\phi)+\mu^{i}+Z-z^{i}\right)+\phi \\
\frac{\partial^{2} \mathcal{E}\left(\phi, Z, z^{i}, \mu^{i}\right)}{\partial^{2} \mu^{i}}=\frac{2(n-1)^{2}}{n^{2}} \alpha_{3}+2 \kappa_{0}, \\
\frac{\partial^{2} \mathcal{E}\left(\phi, Z, z^{i}, \mu^{i}\right)}{\partial \mu^{i} \partial \phi}=-2 \kappa_{0} n \beta^{\prime}(\phi)+1, \\
\frac{\partial^{2} \mathcal{E}\left(\phi, Z, z^{i}, \mu^{i}\right)}{\partial^{2} \phi}=2 \kappa_{0} n^{2}\left(\beta^{\prime}(\phi)\right)^{2}-2 \beta^{\prime}(\phi)+\frac{1}{2 \kappa_{0} n^{2}} \\
=\frac{1}{2 \kappa_{0} n^{2}}\left(1-2 n^{2} \kappa_{0} \beta^{\prime}(\phi)\right)^{2} .
\end{gathered}
$$

We have that $\kappa_{0}<0$ by assumption, and we see from equation (65) that $\alpha_{3} \leq-\gamma /(r(n-1))$. It follows that

$$
\frac{\partial^{2} \mathcal{E}\left(\phi, Z, z^{i}, \mu^{i}\right)}{\partial^{2} \mu^{i}}<0
$$

Now, define

$$
\Theta \equiv 2 \kappa_{0} n \beta^{\prime}(\phi)=\frac{-r \kappa_{0} n}{\gamma}+\frac{1}{n}>\frac{1}{n}
$$

Noting that

$$
\frac{\partial \Phi_{(a, b, c)}\left(D ; Z-z^{i}\right)}{\partial D}=\frac{-1}{b(n-1)}>0
$$

we see that

$$
\begin{aligned}
\frac{\partial^{2} \mathcal{E}\left(\Phi_{(a, b, c)}\left(D ; Z-z^{i}\right), Z, z^{i}, \mu^{i}\right)}{\partial^{2} D} & =\frac{\partial^{2} \mathcal{E}\left(\phi, Z, z^{i}, \mu^{i}\right)}{\partial^{2} \phi}\left(\frac{-1}{b(n-1)}\right)^{2} \\
& =\frac{1}{2 \kappa_{0} n^{2}}\left(1-2 n^{2} \kappa_{0} \beta^{\prime}(\phi)\right)^{2}\left(\frac{-1}{b(n-1)}\right)^{2}<0 .
\end{aligned}
$$


The full optimization is

$$
\sup _{d, \mu^{i}} Q\left(d, \mu^{i}, z, Z\right)
$$

where

$$
Q\left(d, \mu^{i}, z^{i}, Z\right)=-\Phi_{(a, b, c)}\left(d ; Z-z^{i}\right) d+V_{z}\left(z^{i}, Z\right) d+\lambda \mathcal{E}\left(\Phi_{(a, b, c)}\left(d ; Z-z^{i}\right), Z, z^{i}, \mu^{i}\right) .
$$

It is clear from the above that

$$
\frac{\partial^{2} Q\left(d, \mu^{i}, z^{i}, Z\right)}{\partial^{2} \mu^{i}}=\lambda \frac{\partial^{2} \mathcal{E}\left(\Phi_{(a, b, c)}\left(d ; Z-z^{i}\right), Z, z^{i}, \mu^{i}\right)}{\partial^{2} \mu^{i}}<0 .
$$

We also have that

$$
\begin{aligned}
\frac{\partial^{2} Q\left(d, \mu^{i}, z^{i}, Z\right)}{\partial^{2} d} & =-2 \Phi_{(a, b, c)}^{\prime}\left(d ; Z-z^{i}\right)+\lambda \frac{\partial^{2} \mathcal{E}\left(\Phi_{(a, b, c)}\left(d ; Z-z^{i}\right), Z, z^{i}, \mu^{i}\right)}{\partial^{2} d} \\
& =\frac{2}{b(n-1)}+\lambda\left(\frac{1}{-b(n-1)}\right)^{2} \frac{\partial^{2} \mathcal{E}\left(\phi, Z, z^{i}, \mu^{i}\right)}{\partial^{2} \phi} \\
& =\frac{2}{b(n-1)}+\lambda\left(\frac{1}{-b(n-1)}\right)^{2} \frac{1}{2 \kappa_{0} n^{2}}\left(1-2 n^{2} \kappa_{0} \beta^{\prime}(\phi)\right)^{2}<0 .
\end{aligned}
$$

We now prove that the second-order condition for a maximum holds at the optimum: the quantity

$$
\left(\frac{\partial^{2} Q\left(D, \mu^{i}, z^{i}, Z\right)}{\partial^{2} D}\right)\left(\frac{\partial^{2} Q\left(D, \mu^{i}, z^{i}, Z\right)}{\partial^{2} \mu^{2}}\right)-\left(\frac{\partial^{2} Q\left(D, \mu^{i}, z^{i}, Z\right)}{\partial D \partial \mu^{i}}\right)^{2}
$$

is positive. First, note that

$$
\begin{aligned}
\frac{\partial^{2} Q\left(D, \mu^{i}, z^{i}, Z\right)}{\partial D \partial \mu^{i}} & =\lambda \frac{\partial^{2} \mathcal{E}\left(\Phi_{(a, b, c)}\left(D ; Z-z^{i}\right), Z, z^{i}, \mu^{i}\right)}{\partial D \partial \mu^{i}} \\
& =\lambda \Phi_{(a, b, c)}^{\prime}\left(D ; Z-z^{i}\right) \frac{\partial^{2} \mathcal{E}\left(\phi, Z, z^{i}, \mu^{i}\right)}{\partial \phi \partial \mu^{i}} \\
& =\frac{\lambda}{-b(n-1)}(1-\Theta) .
\end{aligned}
$$

From the above,

$$
\begin{aligned}
\frac{\partial^{2} Q\left(D, \mu^{i}, z^{i}, Z\right)}{\partial^{2} \mu^{i}} & =\lambda \frac{\partial^{2} \mathcal{E}\left(\Phi_{(a, b, c)}\left(D ; Z-z^{i}\right), Z, z^{i}, \mu^{i}\right)}{\partial^{2} \mu^{i}} \\
& =\lambda\left(\frac{2(n-1)^{2}}{n^{2}} \alpha_{3}+2 \kappa_{0}\right),
\end{aligned}
$$


so this is equivalent to showing that

$$
\begin{aligned}
& \left(\frac{2}{b(n-1)}+\lambda\left(\frac{1}{-b(n-1)}\right)^{2} \frac{1}{2 \kappa_{0} n^{2}}\left(1-2 n^{2} \kappa_{0} \beta^{\prime}(\phi)\right)^{2}\right)\left(\lambda\left(\frac{2(n-1)^{2}}{n^{2}} \alpha_{3}+2 \kappa_{0}\right)\right) \\
& -\left(\frac{\lambda}{-b(n-1)}(1-\Theta)\right)^{2}>0 .
\end{aligned}
$$

Pulling out $\lambda^{2} /(b(n-1))^{2}$ which is positive, this has the same sign as

$$
\begin{aligned}
& \left(\frac{2 b(n-1)}{\lambda}+\frac{1}{2 \kappa_{0} n^{2}}\left(1-2 n^{2} \kappa_{0} \beta^{\prime}(\phi)\right)^{2}\right)\left(\left(\frac{2(n-1)^{2}}{n^{2}} \alpha_{3}+2 \kappa_{0}\right)\right)-(1-\Theta)^{2} \\
& =\left(\frac{2 b(n-1)}{\lambda}+\frac{1}{2 \kappa_{0} n^{2}}(1-n \Theta)^{2}\right)\left(\left(\frac{2(n-1)^{2}}{n^{2}} \alpha_{3}+2 \kappa_{0}\right)\right)-(1-\Theta)^{2} .
\end{aligned}
$$

Now, suppose that $\Theta \leq 1$. We know that $\Theta>1 / n$ by definition, implying that

$$
(1-\Theta)^{2}<\frac{(n-1)^{2}}{n^{2}}
$$

By definition of $\alpha_{3}$ in equation (65),

$$
\frac{2(n-1)^{2}}{n^{2}}\left(\alpha_{3} \frac{2 b(n-1)}{\lambda}\right)>\frac{2(n-1)^{2}}{n^{2}},
$$

and thus

$$
\frac{2(n-1)^{2}}{n^{2}}\left(\alpha_{3} \frac{2 b(n-1)}{\lambda}\right)-(1-\Theta)^{2}>0 .
$$

It follows that the expression (72) is positive since the remaining terms are all positive. Now, suppose that $\Theta>1$. In this case,

$$
\frac{1}{2 \kappa_{0} n^{2}}(1-n \Theta)^{2} 2 \kappa_{0}=\left(\frac{1}{n}-\Theta\right)^{2}>(1-\Theta)^{2}
$$

so that

$$
\frac{1}{2 \kappa_{0} n^{2}}(1-n \Theta)^{2} 2 \kappa_{0}-(1-\Theta)^{2}>0
$$

Again, this implies (72) is positive since all the remaining terms are positive. We have thus shown for all possible values of $\Theta$ that the expression (72) is positive, which means we have confirmed the conjecture that the solution to the first-order conditions maximizes (59).

\section{B.3.2 Individual rationality}

Finally, we must confirm the optimality of participation in the size-discovery sessions. Specifically, we must check that the equilibrium value achieved in the optimization (59) is higher than 
the highest value achievable in the optimization

$$
\sup _{d}-\Phi_{(a, b, c)}\left(d ; Z-z^{i}\right) d+V_{z}\left(z^{i}, Z\right) d
$$

that results from setting $\mu^{i}=\nu$ and not participating in size discovery. We first calculate a simplified expression for the equilibrium value achieved in (59). Recall that

$$
\begin{aligned}
D & =a+b \phi+c z^{i}=c\left(z^{i}-\bar{Z}\right) \\
\phi & =\alpha_{1}+\left(\alpha_{5}+2 \alpha_{3}\right) \bar{Z} \\
V_{z}\left(z^{i}, Z\right) & =\alpha_{1}+2 \alpha_{3} z^{i}+\alpha_{5} \bar{Z} .
\end{aligned}
$$

Thus, on the equilibrium path,

$$
-\Phi_{(a, b, c)}\left(D ; Z-z^{i}\right) D+V_{z}\left(z^{i}, Z\right) D=2 c \alpha_{3}\left(z^{i}-\bar{Z}\right)^{2} .
$$

Also, on the equilibrium path, at each size-discovery session trader $i$ achieves a post-size discovery inventory of $\bar{Z}$. From equation (58), the equilibrium cash transfer is given by

$$
\frac{a+c \bar{Z}}{-b}\left(z^{i}-\bar{Z}\right)=\phi\left(z^{i}-\bar{Z}\right)=\left(\alpha_{1}+\left(\alpha_{5}+2 \alpha_{3}\right) \bar{Z}\right)\left(z^{i}-\bar{Z}\right) .
$$

It follows that on the equilibrium path,

$$
\begin{aligned}
\mathcal{E}\left(\Phi_{(a, b, c)}\left(D ; Z-z^{i}\right), Z, z^{i}, \mu^{i}\right) & =V(\bar{Z}, \bar{Z})-V\left(z^{i}, \bar{Z}\right)+\left(\alpha_{1}+\left(\alpha_{5}+2 \alpha_{3}\right) \bar{Z}\right)\left(z^{i}-\bar{Z}\right) \\
& =\left(\alpha_{1}+\alpha_{5} \bar{Z}\right)\left(\bar{Z}-z^{i}\right)+\alpha_{3} \bar{Z}^{2}-\alpha_{3}\left(z^{i}\right)^{2}-\left(\bar{Z}-z^{i}\right)\left(\alpha_{1}+\left(\alpha_{5}+2 \alpha_{3}\right) \bar{Z}\right) \\
& =-\alpha_{3}\left(z^{i}-\bar{Z}\right)^{2} .
\end{aligned}
$$

Putting this together, the supremized quantity inside the HJB (59) equals

$$
\alpha_{3}(-\lambda+2 c)\left(z^{i}-\bar{Z}\right)^{2}
$$

We now show the optimized value of the HJB (73) corresponding to no size discovery participation is smaller for all $\left(z^{i}, \bar{Z}\right)$. The first-order condition for $(73)$ is

$$
-\Phi_{(a, b, c)}^{\prime}\left(D ; Z-z^{i}\right) D-\Phi_{(a, b, c)}\left(D ; Z-z^{i}\right)+V_{z}\left(z^{i}, Z\right)=0 .
$$

The second-order condition is satisfied since $b<0$ for the equilibrium $b$. Plugging in values, this is

$$
\frac{D}{b(n-1)}+\frac{1}{b(n-1)}\left(D+(n-1) a+c\left(Z-z^{i}\right)\right)+\alpha_{1}+2 \alpha_{3} z^{i}+\alpha_{5} \bar{Z}=0 .
$$

Recall from equation (67) that $\alpha_{1}=-a / b$, so this simplifies to

$$
\frac{1}{b(n-1)}\left(2 D+c\left(Z-z^{i}\right)\right)+2 \alpha_{3} z^{i}+\alpha_{5} \bar{Z}=0 .
$$


Let $\hat{D}$ denote the solution to this first-order condition:

$$
\hat{D}=-\frac{c}{2}\left(Z-z^{i}\right)-\frac{b(n-1)}{2}\left(2 \alpha_{3} z^{i}+\alpha_{5} \bar{Z}\right) .
$$

We see that

$$
\begin{aligned}
\Phi_{(a, b, c)}\left(\hat{D} ; Z-z^{i}\right) & =-\frac{1}{b(n-1)}\left(\hat{D}+(n-1) a+c\left(Z-z^{i}\right)\right) \\
& =-\frac{a}{b}-\frac{c\left(Z-z^{i}\right)}{2 b(n-1)}+\frac{2 \alpha_{3} z^{i}+\alpha_{5} \bar{Z}}{2} .
\end{aligned}
$$

It follows that

$$
\begin{aligned}
V_{z}\left(z^{i}, Z\right)-\Phi_{(a, b, c)}\left(\hat{D} ; Z-z^{i}\right) & =\alpha_{1}+\alpha_{5} \bar{Z}+2 \alpha_{3} z^{i}-\left(-\frac{a}{b}-\frac{c\left(Z-z^{i}\right)}{2 b(n-1)}+\frac{2 \alpha_{3} z^{i}+\alpha_{5} \bar{Z}}{2}\right) \\
& =\alpha_{5} \bar{Z}+2 \alpha_{3} z^{i}-\left(-\frac{c\left(Z-z^{i}\right)}{2 b(n-1)}+\frac{2 \alpha_{3} z^{i}+\alpha_{5} \bar{Z}}{2}\right) \\
& =\frac{\alpha_{5} \bar{Z}+2 \alpha_{3} z^{i}}{2}+\frac{c\left(Z-z^{i}\right)}{2 b(n-1)},
\end{aligned}
$$

and thus

$$
\begin{aligned}
& \left(V_{z}\left(z^{i}, Z\right)-\Phi_{(a, b, c)}\left(\hat{D} ; Z-z^{i}\right)\right) \hat{D} \\
& =\left(\frac{\alpha_{5} \bar{Z}+2 \alpha_{3} z^{i}}{2}+\frac{c\left(Z-z^{i}\right)}{2 b(n-1)}\right)\left(-\frac{c}{2}\left(Z-z^{i}\right)-\frac{b(n-1)}{2}\left(2 \alpha_{3} z^{i}+\alpha_{5} \bar{Z}\right)\right) \\
& =-b(n-1)\left(\frac{\alpha_{5} \bar{Z}+2 \alpha_{3} z^{i}}{2}+\frac{c\left(Z-z^{i}\right)}{2 b(n-1)}\right)^{2} .
\end{aligned}
$$

Now, we use equations (66) and (64) to write

$$
\alpha_{5}+2 \alpha_{3}=-\frac{2 \gamma}{r}=-\frac{c}{b},
$$

so

$$
\begin{aligned}
\frac{c}{b} \frac{Z-z^{i}}{n-1} & =-\left(2 \alpha_{3}+\alpha_{5}\right)\left(\frac{\bar{Z}-z^{i}}{n-1}+\bar{Z}\right) \\
& =\frac{1}{n-1}\left(2 \alpha_{3} z^{i}+\alpha_{5} z^{i}\right)-\left(\alpha_{5} \bar{Z}+2 \alpha_{3} \bar{Z}\right)-\frac{1}{n-1}\left(\alpha_{5} \bar{Z}+2 \alpha_{3} \bar{Z}\right) .
\end{aligned}
$$


It follows that

$$
\begin{aligned}
& -b(n-1)\left(\frac{\alpha_{5} \bar{Z}+2 \alpha_{3} z^{i}}{2}+\frac{c\left(Z-z^{i}\right)}{2 b(n-1)}\right)^{2} \\
& =\frac{-b(n-1)}{4}\left(\alpha_{5} \bar{Z}+2 \alpha_{3} z^{i}+\frac{1}{n-1}\left(2 \alpha_{3}\left(z^{i}-\bar{Z}\right)+\alpha_{5}\left(z^{i}-\bar{Z}\right)\right)-\left(\alpha_{5} \bar{Z}+2 \alpha_{3} \bar{Z}\right)\right)^{2} \\
& =\frac{-b(n-1)}{4}\left(2 \alpha_{3}+\frac{1}{n-1}\left(2 \alpha_{3}+\alpha_{5}\right)\right)^{2}\left(z^{i}-\bar{Z}\right)^{2} .
\end{aligned}
$$

Next, recall from equation (65) that

$$
\alpha_{3}=-\frac{\gamma}{r(n-1)}+\frac{\lambda}{2 b(n-1)} .
$$

It follows that

$$
2 \alpha_{3}+\frac{1}{n-1}\left(2 \alpha_{3}+\alpha_{5}\right)=-\frac{2 \gamma}{r(n-1)}+\frac{\lambda}{b(n-1)}-\frac{2 \gamma}{r(n-1)},
$$

and pulling out $b(n-1)$, this is

$$
\begin{aligned}
& \frac{-1}{4 b(n-1)}\left(-\frac{4 \gamma b}{r}+\lambda\right)^{2}\left(z^{i}-\bar{Z}\right)^{2} \\
& =\frac{-1}{4 b(n-1)}(-2 c+\lambda)^{2}\left(z^{i}-\bar{Z}\right)^{2}
\end{aligned}
$$

Finally, note from the first equality in equation (65) that

$$
\alpha_{3}=\frac{c-\lambda}{-2 b(n-1)}
$$

and thus that

$$
\begin{aligned}
\left|\alpha_{3}\right| & =\left|\frac{c-\lambda}{-2 b(n-1)}\right| \\
& =\left|\frac{2 c-\lambda}{-4 b(n-1)}+\frac{\lambda}{4 b(n-1)}\right| \\
& >\left|\frac{2 c-\lambda}{-4 b(n-1)}\right|,
\end{aligned}
$$

where the last line follows from the fact that both terms inside the absolute value are negative: 
$b<0, c<0$ from the calculations of the previous section. It follows that

$$
\alpha_{3}(2 c-\lambda)>\frac{-1}{4 b(n-1)}(-2 c+\lambda)^{2} .
$$

Since the equilibrium value of the quantity inside the HJB equation (59) is

$$
\alpha_{3}(-\lambda+2 c)\left(z^{i}-\bar{Z}\right)^{2},
$$

and the best achievable value in the HJB (73) corresponding to $\mu^{i}=\nu$ is

$$
\frac{-1}{4 b(n-1)}(-2 c+\lambda)^{2}\left(z^{i}-\bar{Z}\right)^{2},
$$

we have shown that $\mu^{i}=\nu$ is suboptimal, relative to the equilibrium strategy. It follows that $\mu^{i}=z^{i}, D=a+b \phi+c z^{i}$ maximizes the HJB equation in Proposition 5.

In summary, we have shown that for the coefficients $(a, b, c)$ given by (14)-(16), the value function $V^{i}$ specified by (9) solves the HJB equation (51). Moreover, the optimization problems posed in the HJB equation are solved by the exchange demand $d=a+b p+c z^{i}$ for $p=P(\bar{Z})$, and by the individual rationality of participation in size-discovery sessions with the truthful report $\mu^{i}=z^{i}$. This completes the proof of Proposition 5 .

\section{B.4 Verification of optimality of candidate equilibrium strategies}

In this section, we fix $\kappa_{0}<0$ and $\lambda \leq \bar{\lambda}$, as well as demand function coefficients $(a, b, c)$ satisfying the conditions of Proposition 5, including $2 c<r+\lambda$. We fix the corresponding candidate value function $V$ for trader $i$ specified by Proposition 5. We fix some admissible demand process $D$, and report process $\mu$, by which the inventory of trader $i$ at any time $t<\mathcal{T}$, including the jumps that occur at size-discovery sessions, is

$$
\hat{z}_{t}^{D, \mu}=z_{0}^{i}+\int_{0}^{t} D_{s} d s+H_{t}^{i}+\sum_{\left\{k: t_{0}<\tau_{k} \leq t\right\}} Y^{i}\left(\left(\mu_{k}, z_{\tau_{k}}^{-i}\right)\right) .
$$

We define

$$
U_{t}=1_{t<\mathcal{T}} V\left(\hat{z}_{t}^{D, \mu}, Z_{t}\right)+1_{t \geq \mathcal{T}} v z_{t}^{D, \mu} .
$$

For the remainder of this verification proof, we fix the filtration $\left\{\overline{\mathcal{F}}_{t}: t \geq 0\right\}$. Let $\tilde{\mu}$ be the process that is defined on the $k$-th stochastic interval $\left(\tau_{k-1}, \tau_{k}\right]$, for any $k$, by $\tilde{\mu}_{t}=\mu_{k}$, and let $\hat{\mu}$ be the optional projection ${ }^{38}$ of $\tilde{\mu}$. Because $\hat{\mu}\left(\tau_{k}\right)=\mu_{k}$, we can re-write the sum

$$
\sum_{\left\{k: t_{0}<\tau_{k} \leq \mathcal{T}\right\}} T_{Q}^{i}\left(\left(\mu_{k}, z_{\tau_{k}}^{-i}\right), \Phi_{(a, b, c)}\left(D_{\tau_{k}} ; Z_{\tau_{k}}-z_{\tau_{k}}^{D, \mu}\right)\right)
$$

\footnotetext{
${ }^{38}$ The intuition is that $\hat{\mu}_{t}$ is essentially the same, for $t \in\left(\tau_{k-1}, \tau_{k}\right]$, as $E\left(\mu_{k} \mid \overline{\mathcal{F}}_{t}\right)$. See Protter (2005), pages 367-369.
} 
as the integral

$$
\int_{0}^{\mathcal{T}} T_{Q}^{i}\left(\left(\hat{\mu}_{t}, z_{t}^{-i}\right), \Phi_{(a, b, c)}\left(D_{t} ; Z_{t}-z_{t}^{D, \mu}\right)\right) d N_{t}
$$

and likewise for $\sum_{\left\{k: t_{0}<\tau_{k}<\mathcal{T}\right\}} Y^{i}\left(\left(\mu_{k}, z_{\tau_{k}}^{-i}\right)\right)$.

Following the steps of the derivation of the value function, Itô's Formula implies that

$$
\mathbb{E}\left(U_{\mathcal{T}}-U_{0}\right)=\mathbb{E}\left(\int_{0}^{\mathcal{T}} \tilde{\zeta}_{s} d s\right)
$$

where $^{39}$

$$
\begin{aligned}
\tilde{\zeta}_{s}=D_{s} & \left(\alpha_{1}+\alpha_{5} \bar{Z}_{s}+2 \alpha_{3} z_{s}^{D, \mu}\right)+\alpha_{4} \frac{\sigma_{Z}^{2}}{n^{2}}+\alpha_{3} \sigma_{i}^{2}+\alpha_{5} \frac{\rho^{i}}{n} \\
& +\lambda Y^{i}\left(\left(\hat{\mu}_{s}, z_{s}^{-i}\right)\right)\left(\alpha_{1}+\alpha_{5} \bar{Z}_{s}+2 \alpha_{3} z_{s}^{D, \mu}+\alpha_{3} Y^{i}\left(\left(\hat{\mu}_{s}, z_{s}^{-i}\right)\right)\right)+r\left(v z_{s}^{D, \mu}-V\left(z_{s}^{D, \mu}, Z_{s}\right)\right) .
\end{aligned}
$$

Because $V$ satisfies the HJB equation (51), we have

$$
\begin{aligned}
\mathbb{E}\left(U_{\mathcal{T}}-U_{0}\right) \leq \mathbb{E} & {\left[\int_{0}^{\mathcal{T}} D_{s} \Phi_{(a, b, c)}\left(D_{s} ; Z_{s}-z_{s}^{D, \mu}\right)+\gamma\left(z_{s}^{D, \mu}\right)^{2} d s\right] } \\
& -\mathbb{E}\left[\int_{0}^{\mathcal{T}} T_{Q}^{i}\left(\left(\hat{\mu}_{s}, z_{s}^{-i}\right) ; \Phi_{(a, b, c)}\left(D_{s} ; Z_{s}-z_{s}^{D, \mu}\right)\right) d N_{s}\right] .
\end{aligned}
$$

Rearranging,

$$
\begin{aligned}
V\left(z_{0}^{i}, Z_{0}\right) \geq \mathbb{E} & {\left[\pi z_{\mathcal{T}}^{D, \mu}+\int_{0}^{\mathcal{T}}-D_{s} \Phi_{(a, b, c)}\left(D_{s} ; Z_{s}-z_{s}^{D, \mu}\right)-\gamma\left(z_{s}^{D, \mu}\right)^{2} d s\right] } \\
+ & \mathbb{E}\left[\int_{0}^{\mathcal{T}} T_{Q}^{i}\left(\left(\hat{\mu}_{s}, z_{s}^{-i}\right) ; \Phi_{(a, b, c)}\left(D_{s} ; Z_{s}-z_{s}^{D, \mu}\right)\right) d N_{s}\right] \\
=\mathbb{E} & {\left[\pi z_{\mathcal{T}}^{D, \mu}+\int_{0}^{\mathcal{T}}-D_{s} \Phi_{(a, b, c)}\left(D_{s} ; Z_{s}-z_{s}^{D, \mu}\right)-\gamma\left(z_{s}^{D, \mu}\right)^{2} d s\right] } \\
+ & \mathbb{E}\left[\sum_{\left\{k: t_{0}<\tau_{k}<\mathcal{T}\right\}} T_{Q}^{i}\left(\left(\mu_{k}, z_{\tau_{k}}^{-i}\right), \Phi_{(a, b, c)}\left(D_{\tau_{k}} ; Z_{\tau_{k}}-z_{\tau_{k}}^{D, \mu}\right)\right)\right] .
\end{aligned}
$$

Because $V$ satisfies the HJB equation (51), this relationship holds with equality for the conjectured equilibrium strategy, so this conjectured equilibrium strategy is optimal and $V$ is indeed the value function, as proposed by Proposition 5. Combining with the results of the previous subsection, this completes the proof of Proposition 1.

\footnotetext{
${ }^{39}$ In the definition of $\tilde{\zeta}$, we have used the fact that $\hat{z}^{D, \mu}=z^{D, \mu}$ for almost every $(\omega, t)$, so that $\hat{z}^{D, \mu}$ and $z^{D, \mu}$ can be used interchangeably without affecting $\mathbb{E}\left(\int_{0}^{\mathcal{T}} \tilde{\zeta}_{s} d s\right)$.
} 


\section{Proof of Proposition 2}

In this section, we provide a proof of Proposition 2, showing that the dark-pool size-discovery mechanism leads to the same equilibria as the linear-quadratic mechanism. We take the setup of Proposition 1, but consider size-discovery transfer and reallocation functions defined in Section 3.5.1.

\section{C.1 Potential equilibrium value functions}

In this section, we characterize the potential equilibrium value functions. In any equilibrium, each trader submits an affine demand function of the form (1) for some coefficients $(a, b, c)$ :

$$
D^{i}(\omega, t, p)=a+b p+c z_{t}^{i}(\omega) .
$$

It follows that in any equilibrium, the exchange price must be

$$
\phi_{t}=\frac{a+c \bar{Z}_{t}}{-b}
$$

Also, by our equilibrium definition, in any equilibrium the dark-pool mechanism produces the efficient allocation, so the mechanism allocates $\bar{Z}-z^{i}$ units of the asset to each trader. The dark-pool cash transfer function is $T_{D}^{i}(\mu, p)=-p Y^{i}(\mu)$, so combining these facts, in equilibrium the mechanism cash transfer must equal

$$
\left(\frac{a+c \bar{Z}_{t}}{-b}\right)\left(z^{i}-\bar{Z}\right) .
$$

From equation (58), we see this implies that for any fixed $(a, b, c)$, the equilibrium cash transfers are the same for this dark-pool mechanism as they are for the linear-quadratic mechanism $\left(T_{Q}, Y\right)$. In particular, the equilibrium dark-pool cash transfers can be written as

$$
R_{0}+R_{1} Z_{t}+R_{2} Z_{t}^{2}+R_{3} Z_{t} z_{t}^{i}+R_{4} z_{t}^{i}
$$

for the same same coefficients

$$
\begin{aligned}
R_{0} & =0 \\
R_{1} & =\frac{a}{n b} \\
R_{2} & =\frac{c}{n^{2} b} \\
R_{3} & =\frac{c}{-n b} \\
R_{4} & =\frac{a}{-b} .
\end{aligned}
$$

We note that this fact does not rely on the exact form of $Y^{i}$. Applying Lemma 3, for any $(a, b, c)$ the candidate equilibrium value functions for the dark-pool mechanism are thus exactly the same as the candidate equilibrium value functions for the linear-quadratic mechanism. It 
just remains to show that the only $(a, b, c)$ consistent with equilibrium are those stated in Proposition 1.

\section{C.2 An intermediate optimization problem}

The following lemma summarizes a key result for the proof of Proposition 2.

Lemma 4. Fix some candidate coefficients $(a, b, c)$ with $b<0$ and some trader $i$. Let $V(z, Z)$ denote the linear-quadratic equilibrium value function for trader $i$ implied by the coefficients $(a, b, c)$ by Lemma 3. Then the solution $\left(D_{*}, y_{*}^{i}\right)$ to

$\sup _{D, y^{i}}\left\{-\Phi_{(a, b, c)}\left(D ; Z-z^{i}\right) D+V_{z}\left(z^{i}, Z\right) D+\lambda\left(V\left(z^{i}+y^{i}, Z\right)-V\left(z^{i}, Z\right)-\Phi_{(a, b, c)}\left(D ; Z-z^{i}\right) y^{i}\right)\right\}$

satisfies

$$
\begin{aligned}
D_{*} & =a+b \Phi_{(a, b, c)}\left(D_{*} ; Z-z^{i}\right)+c z^{i} \\
y_{*}^{i} & =\bar{Z}-z^{i},
\end{aligned}
$$

for all $z^{i}, Z$ if and only if $(a, b, c)$ are those given in Proposition 1. If $(a, b, c)$ are those given in Proposition 1, the solution $\left(D_{*}, y_{*}^{i}\right)$ is unique.

Proof: Fix coefficients $(a, b, c)$ with $b<0$ and a trader $i$. First, note the first derivative of the above objective with respect to $y^{i}$ is

$$
\lambda\left(V_{z}\left(z^{i}+y^{i}, Z\right)-\Phi_{(a, b, c)}\left(D ; Z-z^{i}\right)\right),
$$

while the second derivative is $\lambda V_{z z}\left(z^{i}+y^{i}, Z\right)$. The first derivative with respect to $D$ is

$$
-\Phi_{(a, b, c)}^{\prime}\left(D ; Z-z^{i}\right) D-\Phi_{(a, b, c)}\left(D ; Z-z^{i}\right)+V_{z}\left(z^{i}, Z\right)-\lambda \Phi_{(a, b, c)}^{\prime}\left(D ; Z-z^{i}\right) y^{i}
$$

while the second derivative is $-2 \Phi_{(a, b, c)}^{\prime}\left(D ; Z-z^{i}\right)$. Finally, the cross partial is

$$
-\lambda \Phi_{(a, b, c)}^{\prime}\left(D ; Z-z^{i}\right) .
$$

The Hessian of the objective is thus

$$
\left[\begin{array}{cc}
\lambda V_{z z}\left(z^{i}, Z\right) & -\lambda \Phi_{(a, b, c)}^{\prime}\left(D ; Z-z^{i}\right) \\
-\lambda \Phi_{(a, b, c)}^{\prime}\left(D ; Z-z^{i}\right) & -2 \Phi_{(a, b, c)}^{\prime}\left(D ; Z-z^{i}\right)
\end{array}\right] .
$$

Now, suppose that $D_{*}, y_{*}^{i}$ satisfy (77). Note that

$$
D=a+b \Phi_{(a, b, c)}\left(D ; Z-z^{i}\right)+c z^{i}
$$

if and only if

$$
D=a+b \frac{-1}{b(n-1)}\left(D+(n-1) a+c Z^{-j}\right)+c z^{i}
$$


which holds if and only if

$$
b \Phi_{(a, b, c)}\left(D ; Z-z^{i}\right)=b \frac{-1}{b(n-1)}\left(a+b \Phi_{(a, b, c)}\left(D ; Z-z^{i}\right)+c z^{i}+(n-1) a+c Z^{-j}\right)
$$

which in turn holds if and only if

$$
\Phi_{(a, b, c)}\left(D ; Z-z^{i}\right)=\frac{a+c \bar{Z}}{-b} .
$$

Now, since the objective is differentiable, the optimal $D_{*}, y_{*}^{i}$ must satisfy the first-order conditions implied by the derivatives (78), (79). Plugging in the above equation and the valuefunction coefficients $\alpha_{1}$ through $\alpha_{5}$ implied by the coefficients $(a, b, c)$ according to Lemma 3 , we can write the first-order condition implied by (78) as

$$
V_{z}(\bar{Z}, Z)=2 \alpha_{3} \bar{Z}+\alpha_{1}+\alpha_{5} \bar{Z}=\frac{a+c \bar{Z}}{-b} .
$$

Rearranging, this holds if and only if

$$
\begin{aligned}
\alpha_{1} & =\frac{a}{-b} \\
2 \alpha_{3}+\alpha_{5} & =\frac{c}{-b} .
\end{aligned}
$$

Likewise, plugging in the value-function coefficients $\alpha_{1}$ through $\alpha_{5}$ implied by the coefficients $(a, b, c)$ according to Lemma 3 , the derivative in equation (79) can be rearranged as

$$
\begin{gathered}
-\Phi_{(a, b, c)}^{\prime}\left(D ; Z-z^{i}\right) D-\Phi_{(a, b, c)}\left(D ; Z-z^{i}\right)+V_{z}\left(z^{i}, Z\right)-\lambda \Phi_{(a, b, c)}^{\prime}\left(D ; Z-z^{i}\right) y^{i} \\
=\frac{D}{b(n-1)}-\frac{a+c \bar{Z}}{-b}+2 \alpha_{3} z^{i}+\alpha_{1}+\alpha_{5} \bar{Z}+\frac{\lambda\left(\bar{Z}-z^{i}\right)}{b(n-1)} .
\end{gathered}
$$

Plugging in the above conditions (80)-(81), this derivative is equal to

$$
\frac{D}{b(n-1)}+2 \alpha_{3}\left(z^{i}-\bar{Z}\right)+\frac{\lambda\left(\bar{Z}-z^{i}\right)}{b(n-1)} .
$$

Using the fact that $a+b \phi+c z^{i}=c\left(z^{i}-\bar{Z}\right)$ in equilibrium (see equation $(75)$ ), this is equal to

$$
\left(z^{i}-\bar{Z}\right)\left(\frac{c}{b(n-1)}+2 \alpha_{3}-\frac{\lambda}{b(n-1)}\right) .
$$

The first-order condition implied by equation (79) thus holds if and only if

$$
c=-2 \alpha_{3} b(n-1)+\lambda \text {. }
$$


Now, from the derivation of the linear-quadratic value function in Lemma 3, we have

$$
\alpha_{5}=\frac{1}{r+\lambda-c}\left(\frac{c^{2}}{b}-2 \alpha_{3} c+n \lambda R_{3}\right) .
$$

From the last section, in equilibrium we have $R_{3}=c /(-n b)$ and thus

$$
\alpha_{5}=\frac{1}{r+\lambda-c}\left(\frac{c^{2}}{b}-2 \alpha_{3} c-\frac{\lambda c}{b}\right) .
$$

Multiplying both sides by $b(n-1)$, we have

$$
\alpha_{5} b(n-1)=\frac{1}{r+\lambda-c}\left(c^{2}(n-1)-2 \alpha_{3} b(n-1) c-\lambda c(n-1)\right) .
$$

Plugging in equation (82),

$$
\alpha_{5} b(n-1)=\frac{n c}{r+\lambda-c}(c-\lambda) .
$$

Multiplying both sides of (81) by $b(n-1)$,

$$
2 \alpha_{3} b(n-1)+\alpha_{5} b(n-1)=-c(n-1),
$$

and plugging in equations (82) and (83),

$$
\lambda+\frac{n c}{r+\lambda-c}(c-\lambda)+c(n-2)=0 .
$$

This is precisely the same equation for $c$ as that in the proof of Proposition 1. Thus, if $D_{*}, y_{*}^{i}$ satisfy (77), then $c$ is one of the two values from Proposition 1. By Lemma 3, each value of $c$ implies a particular value of $\alpha_{3}$ (the same as that shown in Proposition 1). By equation (82), any $\left(c, \alpha_{3}\right)$ pair implies a particular value of $b$, and by equation (63) this must be the same value for $b$ as in Proposition 1. By Lemma 3,

$$
\alpha_{1}=\frac{1}{r+\lambda-c}\left(r v+\frac{a c}{b}+\lambda R_{4}\right),
$$

where, based on the transfers, $R_{4}=-a / b$. Thus,

$$
\alpha_{1}=\frac{1}{r+\lambda-c}\left(r v+\frac{a c}{b}-\frac{a \lambda}{b}\right) .
$$

Plugging in equation (80), which also holds in the proof of Proposition 1 (see equation (67)),

$$
\frac{a}{-b}=\frac{1}{r+\lambda-c}\left(r v+\frac{a c}{b}-\frac{a \lambda}{b}\right),
$$

which implies a unique value of $a$ for any given $b, c$. In summary, if $D_{*}, y_{*}^{i}$ satisfy (77), then $c$ must be one of the two values from Proposition 1 , and $b, a$ must be the corresponding values from Proposition 1. 
Next, suppose that $(a, b, c)$ are as specified by Proposition 1. Going backward through the same steps, this means the first-order conditions are satisfied by the values $D_{*}, y_{*}^{i}$ satisfying (77). We rewrite the Hessian as

$$
\left[\begin{array}{cc}
\lambda \alpha_{3} & \frac{\lambda}{b(n-1)} \\
\frac{\lambda}{b(n-1)} & \frac{2}{b(n-1)}
\end{array}\right]
$$

which has determinant

$$
\frac{\lambda 2 \alpha_{3}}{b(n-1)}-\frac{\lambda^{2}}{b^{2}(n-1)^{2}}
$$

The determinant has the same sign as

$$
2 \alpha_{3} b(n-1)-\lambda
$$

If condition (82) holds, this in turn has the same sign as $-c$. Since we've assumed a value of $c$ from Proposition 1, we have that the determinant of the Hessian is strictly positive. Because the value of $c$ specified in Proposition 1 implies the negative value of $\alpha_{3}$ from Proposition 1 , the trace is negative. Under these conditions, the Hessian is negative definite everywhere and the values $D_{*}, y_{*}^{i}$ satisfying (77) produce the unique local maximum in the objective.

\section{C.3 Completing the proof}

In this subsection, we characterize the set of demand coefficients $(a, b, c)$ with the property that the corresponding candidate equilibrium value functions given by Lemma 3 satisfy the HJB equation characterizing optimality. Fix some candidate coefficients $(a, b, c)$. Fixing trader $i$ and assuming all traders other than trader $i$ use the equilibrium strategy corresponding to $(a, b, c)$, the HJB equation of trader $i$ is

$$
\begin{aligned}
0=- & \gamma\left(z^{i}\right)^{2}+r\left(v z^{i}-V\left(z^{i}, Z\right)\right)+\frac{\sigma_{i}^{2}}{2} V_{z z}\left(z^{i}, Z\right)+\frac{\sigma_{Z}^{2}}{2} V_{Z Z}\left(z^{i}, Z\right)+\rho^{i} V_{z Z}\left(z^{i}, Z\right) \\
& +\sup _{D, \mu}\left\{-\Phi_{(a, b, c)}\left(D ; Z-z^{i}\right) D+V_{z}\left(z^{i}, Z\right) D+\lambda \Theta\right\}
\end{aligned}
$$

where

$$
\Theta=V\left(z^{i}+Y^{i}(\mu), Z\right)-V\left(z^{i}, Z\right)-\Phi_{(a, b, c)}\left(D ; Z-z^{i}\right) Y^{i}(\mu) .
$$

We claim that if $(a, b, c)$ take the values proposed by Proposition 1, then the corresponding equilibrium strategy solves the optimization in this HJB equation. Suppose, for a proof by contradiction, that this were false. Then there exists some $D^{\prime}, \mu^{\prime}$ such that the objective

$-\Phi_{(a, b, c)}\left(D^{\prime} ; Z-z^{i}\right) D^{\prime}+V_{z}\left(z^{i}, Z\right) D^{\prime}+\lambda\left(V\left(z^{i}+Y^{i}\left(\mu^{\prime}\right), Z\right)-V\left(z^{i}, Z\right)-\Phi_{(a, b, c)}\left(D^{\prime} ; Z-z^{i}\right) Y^{i}\left(\mu^{\prime}\right)\right)$

is larger than the value corresponding to the equilibrium strategy. But at the equilibrium strategy, $Y^{i}(\mu)=\bar{Z}-z^{i}$, so this contradicts Lemma 4. Since, at the values $(a, b, c)$ from Proposition 1, the HJB optimization problem is solved by the equilibrium strategy, we can 
reduce the HJB equation to

$$
\begin{aligned}
0 & =-\gamma\left(z^{i}\right)^{2}+r\left(v z^{i}-V\left(z^{i}, Z\right)\right)+\frac{\sigma_{i}^{2}}{2} V_{z z}\left(z^{i}, Z\right)+\frac{\sigma_{Z}^{2}}{2} V_{Z Z}\left(z^{i}, Z\right)+\rho^{i} V_{z Z}\left(z^{i}, Z\right) \\
& -\Phi_{(a, b, c)}\left(D^{*} ; Z-z^{i}\right) D^{*}+V_{z}\left(z^{i}, Z\right) D^{*}+\lambda\left(V(\bar{Z}, Z)-V\left(z^{i}, Z\right)-\Phi_{(a, b, c)}\left(D^{*} ; Z-z^{i}\right) \mu^{*}\right) .
\end{aligned}
$$

Since the equilibrium size-discovery transfers in Proposition 1 are also equal to

$$
-\Phi_{(a, b, c)}\left(D^{*} ; Z-z^{i}\right) \mu^{*}
$$

by equation (58), this is the same HJB equation as that of Proposition 1. The same verification argument thus shows that the candidate equilibrium strategy is optimal.

Finally, suppose the fixed $(a, b, c)$ values are not those from Proposition 1 . Then by Lemma 4 , there exists some $D^{\prime}, y^{\prime}$ such that the objective

$$
-\Phi_{(a, b, c)}\left(D^{\prime} ; Z-z^{i}\right) D^{\prime}+V_{z}\left(z^{i}, Z\right) D^{\prime}+\lambda\left(V\left(z^{i}+y^{\prime}, Z\right)-V\left(z^{i}, Z\right)-\Phi_{(a, b, c)}\left(D^{\prime} ; Z-z^{i}\right) y^{\prime}\right)
$$

is higher than that achieved by the candidate equilibrium strategy. Since, fixing the equilibrium truth-telling report of any trader $i \neq j$, the function $Y^{i}(\mu)$ is surjective, there exists a report $\mu^{\prime}$ with $Y^{i}\left(\left(\mu^{\prime}, z^{-i}\right)\right)=y^{\prime}$ such that the objective

$$
\begin{aligned}
& -\Phi_{(a, b, c)}\left(D^{\prime} ; Z-z^{i}\right) D^{\prime}+V_{z}\left(z^{i}, Z\right) D^{\prime} \\
& \quad+\lambda\left(V\left(z^{i}+Y^{i}\left(\left(\mu^{\prime}, z^{-i}\right)\right), Z\right)-V\left(z^{i}, Z\right)-\Phi_{(a, b, c)}\left(D^{\prime} ; Z-z^{i}\right) Y^{i}\left(\left(\mu^{\prime}, z^{-i}\right)\right)\right)
\end{aligned}
$$

is higher than that achieved by the candidate equilibrium strategy. Thus, if $(a, b, c)$ are not as specified by Proposition 1, the candidate equilibrium strategy does not solve the HJB equation. Since the candidate equilibrium value function is twice-continuously differentiable, standard verification arguments show the HJB equation is a necessary condition for optimality. It follows that the candidate equilibrium is not an equilibrium, completing the proof.

\section{C.4 Extension to proportional dark-pool rationing}

Here, we extend all of our conclusions about the equilibrium properties of augmenting exchange trading with size discovery to the commonly used dark-pool size-discovery mechanism defined by proportional rationing of heavy-side order submissions (Zhu, 2014).

In the last proof, we did not actually use the form of the size-discovery allocation function $Y^{i}$, beyond the property that if each trader truthfully reports $\mu^{i}=z^{i}$, then the reallocation function $Y^{i}$ generates the efficient allocation. We can therefore adapt all of the arguments used in our demonstration of equilibrium for Proposition 1 to the size-discovery design in which the asset reallocation function is instead $Y_{P}$, defined by

$$
Y_{P}^{i}(\mu) \equiv \mu^{i}\left(\mathbf{1}_{\left\{\mu^{i} \sum_{j} \mu^{j} \leq 0\right\}}+\mathbf{1}_{\left\{\mu^{i} \sum_{j} \mu^{j}>0\right\}}\left|\frac{\sum_{j: \mu^{i} \mu^{j} \leq 0} \mu^{j}}{\sum_{j: \mu^{i} \mu^{j}>0} \mu^{j}}\right|\right) .
$$

This reallocation function $Y_{P}$ represents proportional rationing, as used in practice in stan- 
dard dark pools. For example, if trader $i$ reports a request for $\mu^{i} \geq 0$ units of the asset and if the total net requested demand is weakly negative, in that $\sum_{j} \mu^{j} \leq 0$, then the order of trader $i$ is entirely filled, with trader $i$ receiving $\mu^{i}$. Likewise, if trader $i$ requests $\mu^{i} \leq 0$ units of the asset and the total net demand is weakly positive, in that $\sum_{j} \mu^{j} \geq 0$, then the demand is entirely filled, in that trader $i$ again receives $\mu^{i}$. However, if the trader is on the heavy side, in that $\mu^{i} \neq 0$ shares the same sign as the total demand $\sum_{j} \mu^{j} \neq 0$, then the demand of trader $i$ order is proportionally rationed, matching total sales to total purchases.

With this proportional dark-pool allocation function $Y_{P}$, if each trader $i$ reports the demand $\mu^{i}=\bar{Z}-z^{i}$, the resulting allocation is efficient, the total net demand is zero, and all orders are filled. This proportional-rationing dark-pool size-discovery trade protocol uses the cash allocation function $T_{P}$ analogous to that used for the linear-rationing size-discovery model, defined by

$$
T_{P}^{i}(\mu, p)=-p Y_{P}^{i}(\mu) .
$$

Now, we can define equilibrium for the proportional size-discovery mechanism as a collection of demand coefficients $(a, b, c)$ such that if each trader $j \neq i$ uses the strategy

$$
\begin{aligned}
D^{j}(\omega, t, p) & =a+b p+c z_{t}^{j}(\omega) \\
\mu_{k}^{j} & =\frac{-a-b \phi_{\tau_{k}}}{c}-z_{\tau_{k}}^{j},
\end{aligned}
$$

then trader $i$ finds it optimal to use the same strategy. This equilibrium definition is identical to that for the model whose properties are characterized by Proposition 1, for the cases of linear-rationing dark-pool size discovery and linear-quadratic size discovery, with the exception that for proportional dark-pool rationing traders use the size-discovery report of equation (86) rather than truthful reporting. Given the proportional-rationing asset reallocation function defined above, this leads to efficient reallocations because $\mu_{k}^{j}=\bar{Z}_{\tau_{k}}-z_{\tau_{k}}^{j}$ at the equilibrium price. Because the proof of Proposition 2 did not actually use the specific functional form of $Y_{P}^{i}$, identical arguments used in the proof of equilibrium imply that the coefficients $(a, b, c)$ correspond to an equilibrium in this modified setting if and only if the coefficients are those specified by Proposition 1. Moreover, the resulting allocations and equilibrium value functions are identical to those of Proposition 1.

\section{The model with observable total inventory}

In this appendix, as opposed to situation examined in the main model, the aggregate inventory $Z_{t}$ is assumed to be observable by the size-discovery operator. Our size-discovery sessions use the mechanism design $\left(Y, T_{\kappa}\right)$ of Appendix A, restricting attention to the affine functions $\kappa_{1}(\cdot)$ and $\kappa_{2}(\cdot)$ of $Z_{t}$ that exploit the properties of Propositions 3 and 4 . We will calculate intercept and slope coefficients of both $\kappa_{1}(\cdot)$ and $\kappa_{2}(\cdot)$ that are consistent with the resulting endogenous continuation value functions. In equilibrium, these coefficients imply the transfer function given in Section 3.7. The model setup is otherwise unchanged.

We will show that equilibrium exchange-market demand behavior in this new setting is of the same affine form that we found in the main model, but has different demand coefficients.

The demand process $D^{i}$ and size-discovery reports $\left\{\mu_{1}^{i}, \mu_{2}^{i}, \ldots\right\}$ of each trader $i$ imply that 
the inventory process of trader $i$ is

$$
z_{t}^{i}=z_{0}^{i}+\int_{0}^{t} D_{s}^{i} d s+H_{t}^{i}+\sum_{\left.\left\{k: \tau_{k}<\min (t, \mathcal{T})\right\}\right)} \frac{\sum_{j=1}^{n} \mu_{k}^{j}}{n}-\mu_{k}^{i} .
$$

Our notion of equilibrium implies market clearing, rational conjectures of other traders' strategies, and individual-trader optimality, including the incentive compatibility of truthtelling and individual rationality of participation in all size-discovery sessions. Appendix $\mathrm{F}$ analyzes the discrete-time version of this model, showing that the analogous equilibrium is Perfect Bayes.

The definition of individual-trader optimality in this dynamic game is relatively obvious from the main model, but is now stated for completeness. Taking as given the demand coefficients $(a, b, c)$ used by other traders and the mechanism design $\left(Y, T_{\kappa}\right)$ for size-discovery sessions, trader $i$ faces the problem of choosing a demand process $D^{i}$ and report process $\mu^{i}$ that solve the problem

$$
\sup _{D, \mu} \mathbb{E}_{0}^{i}\left[J_{A}^{i}(D, \mu)\right]
$$

where $\mathbb{E}_{0}^{i}$ denotes expectation conditional on $\mathcal{F}_{0}^{i}$ and

$$
\begin{aligned}
J_{A}^{i}(D, \mu)=z_{\mathcal{T}}^{D, \mu} \pi-\int_{0}^{\mathcal{T}} \gamma\left(z_{t}^{D, \mu}\right)^{2}+\Phi_{(a, b, c)}\left(D_{t} ; Z_{t}-z_{t}^{D, \mu}\right) D_{t} d t \\
\left.+\sum_{\left\{k: \tau_{k}<\mathcal{T}\right\}} T_{\kappa}^{i}\left(\left(\mu_{k}, z_{\tau_{k}}^{-i}\right)\right), Z_{\tau_{k}}\right),
\end{aligned}
$$

where

$$
\begin{aligned}
z_{t}^{j} & \left.=z_{0}^{j}+\int_{0}^{t} \hat{D}_{s}^{j} d s+H_{t}^{j}+\sum_{\left\{k: \tau_{k}<\min (t, \mathcal{T})\right\}} Y^{j}\left(\left(\mu_{k}, z_{\tau_{k}}^{-i}\right)\right), Z_{\tau_{k}}\right), \\
z_{t}^{D, \mu} & \left.=z_{0}^{i}+\int_{0}^{t} D_{s} d s+H_{t}^{i}+\sum_{\left\{k: \tau_{k}<\min (t, \mathcal{T})\right\}} Y^{i}\left(\left(\mu_{k}, z_{\tau_{k}}^{-i}\right)\right), Z_{\tau_{k}}\right),
\end{aligned}
$$

taking $\hat{D}_{t}^{j}=a+b \Phi_{(a, b, c)}\left(D_{t} ; Z_{t}-z_{t}^{D, \mu}\right)+c z_{t}^{j}$. Here again, the equilibrium strategies are ex-post optimal in the sense described in Section 4. That is, in any equilibrium and for any trader $i$, if we relax the information requirement on $(D, \mu)$ by allowing observation of all other traders' excess-inventory positions, trader $i$ would have the same optimal policy.

Proposition 6. Suppose that $\lambda<r(n-2)$. Let $\kappa_{0}<0$ be arbitrary, and fix the mechanism design $\left(Y, T_{\kappa}\right)$ specified by $(25)$ and $(26)$, where

$$
\kappa_{1}\left(Z_{t}\right)=v-\frac{2 \gamma \bar{Z}_{t}}{r}, \quad \kappa_{2}(Z)=-\bar{Z}_{t}-\frac{\kappa_{1}\left(Z_{t}\right)}{2 \kappa_{0} n^{2}} .
$$

1. Among equilibria in the dynamic game associated with the sequential-double-auction market augmented with size-discovery sessions, there is a unique equilibrium with symmetric 
affine double-auction demand functions. In this equilibrium, the double-auction demand function $\mathcal{D}_{t}^{i}$ of trader $i$ in state $\omega$ at time $t$ is given by

$$
\mathcal{D}_{t}^{i}(\omega, p)=\frac{-r \lambda+r^{2}(n-2)}{4 \gamma}\left(v-p-\frac{2 \gamma}{r} z_{t}^{i}(\omega)\right) .
$$

That is, the coefficients $(a, b, c)$ of the demand function are

$$
a=\frac{\left[-r \lambda+r^{2}(n-2)\right] v}{4 \gamma}, \quad b=\frac{r \lambda-r^{2}(n-2)}{4 \gamma}, \quad c=\frac{\lambda-r(n-2)}{2} .
$$

2. The market-clearing double-auction price process $\phi$ is given by $\phi_{t}=\kappa_{1}\left(Z_{t}\right)$.

3. The mechanism design $\left(Y, T_{\kappa}\right)$ achieves the perfect post-session allocation $z^{i}\left(\tau_{k}\right)=\bar{Z}\left(\tau_{k}\right)$ for each trader $i$ at each session time $\tau_{k}$.

4. For each trader $i$, the equilibrium continuation value $V^{i}\left(z_{t}^{i}, Z_{t}\right)$ at time $t$ is invariant to the mean frequency $\lambda$ of size-discovery sessions. In particular, augmenting the exchange market with size discovery has no impact on any trader's value. The value function is given by

$$
V^{i}\left(z_{t}^{i}, Z_{t}\right)=\theta_{i}+v \bar{Z}_{t}-\frac{\gamma}{r} \bar{Z}_{t}^{2}+\left(v-\frac{2 \gamma}{r} \bar{Z}_{t}\right)\left(z_{t}^{i}-\bar{Z}_{t}\right)-K\left(z_{t}^{i}-\bar{Z}_{t}\right)^{2}
$$

where

$$
\begin{gathered}
K=\frac{\gamma}{r(n-1)} \\
\theta_{i}=\frac{1}{r}\left(\frac{\gamma}{r} \frac{\sigma_{Z}^{2}}{n^{2}}-K\left(\frac{\sigma_{Z}^{2}}{n^{2}}+\sigma_{i}^{2}-2 \frac{\rho^{i}}{n}\right)-\frac{2 \gamma}{r} \frac{\rho^{i}}{n}\right) .
\end{gathered}
$$

To prove Proposition 6, we note that that proof of admissibility in Section B carries over exactly to this setting, so a strategy is admissible if and only if $2 c<r+\lambda$. Also, the proof that the value functions must take the linear-quadratic form in equation (47) carries over exactly, although the $R_{0}-R_{4}$ constants might be different. Given the value functions, we use individual rationality to restrict the possible mechanism-transfer coefficients, and characterize the optimal mechanism reports in the equilibrium. Next, we calculate the unique value function and affine coefficients consistent with the HJB equation. Finally, we verify that the candidate value function and these coefficients indeed solve the Markov control problem. Throughout, we write $V(z, Z)$ in place of $V^{i}(z, Z)$.

\section{D.1 The mechanism-transfer coefficients}

Fix a symmetric equilibrium. Recall the mechanism transfers for a session are given by

$$
\kappa_{0}\left(n \kappa_{2}\left(Z_{\tau_{k}}\right)+\sum_{j=1}^{n} \mu_{k}^{j}\right)^{2}+\kappa_{1}\left(Z_{\tau_{k}}\right)\left(\mu_{k}^{i}+\kappa_{2}\left(Z_{\tau_{k}}\right)\right)+\frac{\kappa_{1}^{2}\left(Z_{\tau_{k}}\right)}{4 \kappa_{0} n^{2}} .
$$


For the purpose of this proof, we will treat $\kappa_{1}(\cdot)$ and $\kappa_{2}(\cdot)$ as arbitrary affine functions, and then show the particular choices of $\kappa_{1}(\cdot)$ and $\kappa_{2}(\cdot)$ stated by the proposition are the unique functions consistent with equilibrium. From the above, this transfer function with the conjectured reports leads to a linear-quadratic equilibrium value function $V(z, Z)$. Thus, maximizing $V(z+y, Z)$ with respect to $y$ is equivalent to maximizing

$$
\alpha_{1}\left(z^{i}+y\right)+\alpha_{3}\left(z^{i}+y\right)^{2}+\alpha_{5} \bar{Z}\left(z^{i}+y\right),
$$

which in turn is equivalent to maximizing

$$
\left(\alpha_{1}+\alpha_{5} \bar{Z}+2 \alpha_{3} z^{i}\right) y+\alpha_{3} y^{2}
$$

Then, when trader $i$ chooses a report $\mu_{k}^{i}$, it must be that this choice maximizes, suppressing subscripts from the notation for simplicity,

$$
\left(\alpha_{1}+\alpha_{5} \bar{Z}+2 \alpha_{3} z^{i}\right) Y^{i}\left(\left(\mu, z^{-i}\right)\right)+\alpha_{3} Y^{i}\left(\left(\mu, z^{-i}\right)\right)^{2}+T_{\kappa}^{i}\left(\left(\mu, z^{-i}\right), Z\right) .
$$

The associated first-order condition is

$$
-\frac{n-1}{n}\left(\alpha_{1}+\alpha_{5} \bar{Z}+2 \alpha_{3} z^{i}\right)-\frac{2(n-1) \alpha_{3}}{n} Y^{i}\left(\left(\mu, z^{-i}\right)\right)+\kappa_{1}(Z)+2 \kappa_{0}\left(n \kappa_{2}(Z)+\mu+\sum_{j \neq i} z^{j}\right)=0 .
$$

Plugging in $Y^{i}$, we have

$$
\begin{aligned}
& -\frac{n-1}{n}\left(\alpha_{1}+\alpha_{5} \bar{Z}+2 \alpha_{3} z^{i}\right)-\frac{2(n-1) \alpha_{3}}{n}\left(\frac{-(n-1) \mu}{n}+\frac{Z-z^{i}}{n}\right) \\
& \quad+\kappa_{1}(Z)+2 \kappa_{0}\left(n \kappa_{2}(Z)+\mu-z^{i}+Z\right)=0
\end{aligned}
$$

The second-order condition is satisfied because $\kappa_{0}$ and $\alpha_{3}$ are strictly negative. Since $\kappa_{2}$ is affine, write $\kappa_{2}(Z)=\hat{a}+\hat{b} Z$. The report $\mu=z^{i}$ satisfies this first-order condition if

$$
-\frac{n-1}{n}\left(\alpha_{1}+\alpha_{5} \bar{Z}\right)-\frac{2(n-1) \alpha_{3}}{n} \bar{Z}+\kappa_{1}(Z)+2 \kappa_{0}(n \hat{a}+n \hat{b} Z+Z)=0 .
$$

With this,

$$
(n \hat{a}+n \hat{b} Z+Z)=\frac{\Xi}{2 \kappa_{0}}
$$

where

$$
\Xi=-\kappa_{1}(Z)+\frac{n-1}{n}\left(\alpha_{1}+\left(\alpha_{5}+2 \alpha_{3}\right) \bar{Z}\right)
$$

Thus,

$$
\kappa_{2}(Z)=\hat{a}+\hat{b} Z=-\bar{Z}+\frac{\Xi}{2 \kappa_{0} n},
$$


implying an equilibrium change in utility of

$$
\begin{aligned}
& \frac{\Xi^{2}}{4 \kappa_{0}}+\kappa_{1}(Z)\left(-\bar{Z}+\frac{\Xi}{2 \kappa_{0} n}\right)+\frac{\kappa_{1}^{2}(Z)}{4 n^{2} \kappa_{0}} \\
& +\left(\kappa_{1}(Z)-\alpha_{1}-\alpha_{5} \bar{Z}\right) z^{i}+\left(\alpha_{1}+\alpha_{5} \bar{Z}\right) \bar{Z}-\alpha_{3}\left(z^{i}\right)^{2}+\alpha_{3} \bar{Z}^{2}
\end{aligned}
$$

This change in utility must be weakly positive for any $z$ and $Z$. If all traders have $z=\bar{Z}$, then we need that

$$
\frac{\Xi^{2}}{4 \kappa_{0}}+\kappa_{1}(Z)\left(\frac{\Xi}{2 \kappa_{0} n}\right)+\frac{\kappa_{1}^{2}(Z)}{4 n^{2} \kappa_{0}}=-\left(\frac{\Xi}{2 \sqrt{-\kappa_{0}}}+\frac{\kappa_{1}(Z)}{2 n \sqrt{-\kappa_{0}}}\right)^{2} \geq 0
$$

which implies that $\kappa_{1}(Z)=\alpha_{1}+\left(\alpha_{5}+2 \alpha_{3}\right) \bar{Z}$. Plugging this in, we see that

$$
\hat{a}+\hat{b} Z+z^{i}=z^{i}-\bar{Z}+\frac{\Xi}{2 \kappa_{0} n}=z^{i}-\bar{Z}-\frac{\alpha_{1}+\left(\alpha_{5}+2 \alpha_{3}\right) \bar{Z}}{2 \kappa_{0} n^{2}} .
$$

So, we see that $n \kappa_{2}(Z)+\sum_{j=1}^{n} \hat{z}^{j}=-\left(\alpha_{1}+\left(\alpha_{5}+2 \alpha_{3}\right) \bar{Z}\right) /\left(2 \kappa_{0} n\right)$, and thus the equilibrium transfer to trader $i$ is

$$
\begin{aligned}
& \frac{\left(\alpha_{1}+\left(\alpha_{5}+2 \alpha_{3}\right) \bar{Z}\right)^{2}}{4 n^{2} \kappa_{0}}+\left(\alpha_{1}+\left(\alpha_{5}+2 \alpha_{3}\right) \bar{Z}\right)\left(z^{i}-\bar{Z}-\frac{\left(\alpha_{1}+\left(\alpha_{5}+2 \alpha_{3}\right) \bar{Z}\right)}{2 \kappa_{0} n^{2}}\right) \\
& \quad+\frac{\left(\alpha_{1}+\left(\alpha_{5}+2 \alpha_{3}\right) \bar{Z}\right)^{2}}{4 n^{2} \kappa_{0}} \\
& =\left(\alpha_{1}+\left(\alpha_{5}+2 \alpha_{3}\right) \bar{Z}\right)\left(z^{i}-\bar{Z}\right) .
\end{aligned}
$$

It follows that the equilibrium change in utility for trader $i$ from the mechanism is

$$
\begin{aligned}
\left(\alpha_{1}+\left(\alpha_{5}\right.\right. & \left.\left.+2 \alpha_{3}\right) \bar{Z}\right)\left(z^{i}-\bar{Z}\right)+\left(\alpha_{1}+\alpha_{5} \bar{Z}\right)\left(\bar{Z}-z^{i}\right)+\alpha_{3}(\bar{Z})^{2}-\alpha_{3}\left(z^{i}\right)^{2} \\
& =2 \alpha_{3} \bar{Z} z^{i}-\alpha_{3}(\bar{Z})^{2}-\alpha_{3}\left(z^{i}\right)^{2} \\
& =-\alpha_{3}\left(z^{i}-\bar{Z}\right)^{2} \geq 0
\end{aligned}
$$

where the final inequality relies on the fact that $\alpha_{3}$ is negative in an equilibrium. Putting this together, as long as $\kappa_{1}(Z)=\alpha_{1}+\left(\alpha_{1}+\left(\alpha_{5}+2 \alpha_{3}\right) \bar{Z}\right) \bar{Z}$ and $\kappa_{2}(Z)=\hat{a}+\hat{b} Z$ are given as above, then in equilibrium all traders find the mechanism ex-post individually rational each time it is run, and the strategy $\mu^{i}=z^{i}$ is ex-post optimal. This is true only if $\kappa_{1}(Z)$ and $\kappa_{2}(Z)$ take the specified forms.

Finally, since the equilibrium transfers are $\left(\alpha_{1}+\left(\alpha_{5}+2 \alpha_{3}\right) \bar{Z}\right)\left(z^{i}-\bar{Z}\right)$, we see that the coefficients $\left\{R_{m}\right\}$ in

$$
R_{0}+R_{1} Z_{t}+R_{2} Z_{t}^{2}+R_{3} Z_{t} z_{t}^{i}+R_{4} z_{t}^{i}
$$


are given by

$$
\begin{aligned}
& R_{0}=0 \\
& R_{1}=-\frac{\alpha_{1}}{n} \\
& R_{2}=-\frac{\alpha_{5}+2 \alpha_{3}}{n^{2}} \\
& R_{3}=\frac{\alpha_{5}+2 \alpha_{3}}{n} \\
& R_{4}=\alpha_{1} .
\end{aligned}
$$

Recall from the previous section that

$$
\begin{aligned}
\alpha_{3} & =\frac{-\gamma}{r+\lambda-2 c} \\
\alpha_{5} & =\frac{1}{r+\lambda-c}\left(\frac{c^{2}}{b}-2 \alpha_{3} c+\lambda n R_{3}\right) \\
\alpha_{1} & =\frac{1}{r+\lambda-c}\left(r v+\frac{a c}{b}+\lambda R_{4}\right) .
\end{aligned}
$$

Thus, plugging in $R_{3}, R_{4}$, and rearranging, we have

$$
\begin{aligned}
\alpha_{3} & =\frac{-\gamma}{r+\lambda-2 c} \\
\alpha_{5} & =\frac{1}{r-c}\left(\frac{c^{2}}{b}-2 \alpha_{3} c+2 \lambda \alpha_{3}\right) \\
\alpha_{1} & =\frac{1}{r-c}\left(r v+\frac{a c}{b}\right) .
\end{aligned}
$$

\section{D.2 Solving the HJB equation}

From the above, the value function takes the form

$$
V\left(z^{i}, Z\right)=\alpha_{0}^{i}+\alpha_{1} z^{i}+\alpha_{2} \bar{Z}+\alpha_{3}\left(z^{i}\right)^{2}+\alpha_{4} \bar{Z}^{2}+\alpha_{5} z^{i} \bar{Z} .
$$

The associated HJB equation, analogous to, but simpler than, (51), is

$$
\begin{aligned}
0=- & \gamma\left(z^{i}\right)^{2}+r\left(v z^{i}-V\left(z^{i}, Z\right)\right)+\frac{\sigma_{i}^{2}}{2} V_{z z}\left(z^{i}, Z\right)+\frac{\sigma_{Z}^{2}}{2} V_{Z Z}\left(z^{i}, Z\right)+\rho^{i} V_{z Z}\left(z^{i}, Z\right) \\
& +\sup _{D, \mu}\left\{-\Phi_{(a, b, c)}\left(D ; Z-z^{i}\right) D+V_{z}\left(z^{i}, Z\right) D\right. \\
& \left.+\lambda\left(V\left(z^{i}+Y^{i}\left(\left(\mu, z^{-i}\right)\right), Z\right)-V(z, Z)+T_{\kappa}^{i}\left(\left(\mu^{i}, z^{-i}\right), Z\right)\right)\right\} .
\end{aligned}
$$

From the previous subsection, we know that fixing the truthful candidate equilibrium reports 
$z^{-i}$ of the other traders, the report $\mu=z^{i}$ achieves the supremum in the HJB equation for any $D$, as long as

$$
\kappa_{2}(Z)=\hat{a}+\hat{b} Z=-\bar{Z}-\frac{\alpha_{1}+\left(\alpha_{5}+2 \alpha_{3}\right) \bar{Z}}{2 \kappa_{0} n^{2}} .
$$

Since $V_{z}=\alpha_{1}+2 \alpha_{3} z^{i}+\alpha_{5} \bar{Z}$, we can follow steps identical to those of the proof of Proposition 5 , and see that as long as $b<0$, the unique demand that achieves the maximum in the HJB equation is

$$
D=-\frac{1}{2}\left[(n-1) a+n(-b p-a)-c z^{i}+b(n-1)\left(\alpha_{1}+2 \alpha_{3} z^{i}+\alpha_{5} \bar{Z}\right)\right] .
$$

Plugging in $Z=n(-b p-a) / c$, we have

$$
D=-\frac{1}{2}\left[(n-1) a+n(-b p-a)-c z^{i}+b(n-1)\left(\alpha_{1}+2 \alpha_{3} z^{i}+\alpha_{5} \frac{-b p-a}{c}\right)\right] .
$$

Recall from the previous section that, after plugging in equilibrium transfers,

$$
\begin{aligned}
\alpha_{3} & =\frac{-\gamma}{r+\lambda-2 c} \\
\alpha_{5} & =\frac{1}{r-c}\left(\frac{c^{2}}{b}-2 \alpha_{3} c+2 \lambda \alpha_{3}\right) \\
\alpha_{1} & =\frac{1}{r-c}\left(r v+\frac{a c}{b}\right) .
\end{aligned}
$$

Then, matching coefficients in the expression for $D$, we have

$$
\begin{aligned}
& c=-\frac{1}{2}\left[-c+2 b(n-1) \alpha_{3}\right] \\
& b=-\frac{1}{2}\left[-n b+b(n-1)\left(\frac{1}{r-c}\left[2 \alpha_{3} b-c-\lambda 2 \alpha_{3} \frac{b}{c}\right]\right)\right] \\
& a=-\frac{1}{2}\left[-a+b(n-1) \frac{1}{r-c}\left(r v+2 \lambda \alpha_{3}\left(\frac{-a}{c}\right)+2 \alpha_{3} a\right)\right] .
\end{aligned}
$$


This implies that

$$
\begin{aligned}
c & =-2 b(n-1) \alpha_{3} \\
(r-c)(n-2) & =\left[2 \alpha_{3} b(n-1)-c(n-1)-\lambda 2 \alpha_{3} \frac{b}{c}(n-1)\right] \\
r(n-2) & =-2 c+\lambda \\
c & =\frac{\lambda-r(n-2)}{2} \\
\alpha_{3} & =\frac{-\gamma}{r(n-1)} \\
b & =\frac{r \lambda-r^{2}(n-2)}{4 \gamma} .
\end{aligned}
$$

From this, we see that $b$ is strictly negative, satisfying the second-order condition, if and only if $\lambda<r(n-2)$.

Next, we have

$$
\begin{aligned}
a & =\frac{1}{r-c}\left(-b(n-1) r v+2 \lambda \alpha_{3} b(n-1) \frac{a}{c}-2 \alpha_{3} a b(n-1)\right) \\
& =\frac{1}{r-c}(-b(n-1) r v+-\lambda a+c a) \\
& =\frac{2}{r n-\lambda}\left(-\frac{r \lambda-r^{2}(n-2)}{4 \gamma}(n-1) r v+a \frac{-\lambda-r(n-2)}{2}\right) .
\end{aligned}
$$

Noting that

$$
\frac{\lambda+r(n-2)}{r n-\lambda}+1=\frac{2 r(n-1)}{r n-\lambda},
$$

we see that

$$
a=-\frac{\left(r \lambda-r^{2}(n-2)\right) v}{4 \gamma} .
$$

From this, we see that $a=-v b$ and $c=2 \gamma b / r$, so

$$
\phi_{t}=\frac{a+c \bar{Z}_{t}}{-b}=v-\frac{2 \gamma}{r} \bar{Z}_{t}
$$

and

$$
\begin{aligned}
\alpha_{1} & =\frac{1}{r-c}\left(r v+\frac{a c}{b}\right) \\
& =\frac{1}{r-c}(r v-v c)=v .
\end{aligned}
$$


Likewise,

$$
\begin{aligned}
\alpha_{5}+2 \alpha_{3} & =\frac{1}{r-c}\left(\frac{c^{2}}{b}-2 \alpha_{3} c+2 \lambda \alpha_{3}\right)+2 \alpha_{3} \\
& =\frac{1}{r-c}\left(\frac{2 \gamma}{r} c+2 \alpha_{3}(r-c)-2 \alpha_{3} c+2 \lambda \alpha_{3}\right) \\
& =\frac{1}{r-c}\left(\frac{2 \gamma}{r} c+2 \alpha_{3}(r+\lambda-2 c)\right) \\
& =\frac{1}{r-c}\left(\frac{2 \gamma}{r} c-2 \gamma\right)=\frac{-2 \gamma}{r} .
\end{aligned}
$$

It follows that

$$
\alpha_{5}=\frac{-2 \gamma}{r}-2 \alpha_{3}=\frac{-2 \gamma}{r}+\frac{2 \gamma}{r(n-1)} .
$$

Plugging $\alpha_{1}, \alpha_{5}$, and $\alpha_{3}$ into the equilibrium $\kappa_{2}(Z)$, we see that

$$
\begin{aligned}
& \kappa_{2}(Z)=-\bar{Z}-\frac{\alpha_{1}+\left(\alpha_{5}+2 \alpha_{3}\right) \bar{Z}}{2 \kappa_{0} n^{2}} \\
& \kappa_{2}(Z)=-\bar{Z}-\frac{v-\frac{2 \gamma}{r} \bar{Z}}{2 \kappa_{0} n^{2}} .
\end{aligned}
$$

Likewise,

$$
\kappa_{1}(Z)=\alpha_{1}+\left(\alpha_{5}+2 \alpha_{3}\right) \bar{Z}=v-\frac{2 \gamma}{r} \bar{Z} .
$$

Recalling that $R_{1}=-\alpha_{1} / n$ and $\alpha_{1}=v$, we have

$$
\begin{aligned}
\alpha_{2} & =\frac{1}{r}\left(\frac{c a}{-b}+(\lambda-c) \alpha_{1}+\lambda n R_{1}\right) \\
& =\frac{1}{r}\left(c v+(\lambda-c) v-\lambda \alpha_{1}\right)=0 .
\end{aligned}
$$

Recalling that

$$
R_{2}=-\frac{\alpha_{5}+2 \alpha_{3}}{n^{2}}=\frac{2 \gamma}{r n^{2}}
$$


we have

$$
\begin{aligned}
\alpha_{4} & =\frac{1}{r}\left(\frac{c^{2}}{-b}+(\lambda-c) \alpha_{5}+\lambda \alpha_{3}+\lambda n^{2} R_{2}\right) \\
& =\frac{1}{r}\left(\frac{-2 \gamma}{r} c+(\lambda-c) \alpha_{5}+\lambda \alpha_{3}-\lambda\left(\alpha_{5}+2 \alpha_{3}\right)\right) \\
& =\frac{1}{r}\left(\frac{-2 \gamma}{r} c-c\left(\alpha_{5}+2 \alpha_{3}\right)+(2 c-\lambda) \alpha_{3}\right) \\
& =\frac{1}{r}\left((2 c-\lambda-r) \alpha_{3}+r \alpha_{3}\right) \\
& =\frac{1}{r}\left(\gamma-\frac{\gamma}{(n-1)}\right)=\frac{\gamma(n-2)}{r(n-1)} .
\end{aligned}
$$

Finally, since $R_{0}=0$, we have

$$
\begin{aligned}
\alpha_{0}^{i} & =\frac{1}{r}\left(\alpha_{3} \sigma_{i}^{2}+\alpha_{4} \frac{\sigma_{Z}^{2}}{n^{2}}+\alpha_{5} \frac{\rho^{i}}{n}+\lambda R_{0}\right) \\
& =\frac{1}{r}\left(\alpha_{3} \sigma_{i}^{2}+\alpha_{4} \frac{\sigma_{Z}^{2}}{n^{2}}+\alpha_{5} \frac{\rho^{i}}{n}\right) \\
& =\frac{1}{r}\left(\frac{-\gamma}{r(n-1)} \sigma_{i}^{2}+\left(\frac{\gamma(n-2)}{r(n-1)}\right) \frac{\sigma_{Z}^{2}}{n^{2}}+\left(\frac{-2 \gamma}{r}+\frac{2 \gamma}{r(n-1)}\right) \frac{\rho^{i}}{n}\right),
\end{aligned}
$$

which simplifies to the expression in Proposition 6.

\section{D.3 Completing the verification}

We have shown that in a symmetric equilibrium, the traders' value functions are linear-quadratic and in particular must be twice continuously differentiable. The HJB equation of the previous subsection is thus a necessary condition, and there is a unique candidate linear-quadratic equilibrium that satisfies this equation. We have shown that if each trader follows the suggested affine strategy, they indeed get their candidate value function as a continuation value. It remains to show that each trader prefers this to any other strategy. The verification argument is completely analogous to that associated with the main model, given in Appendix B.4, so simply sketched here for brevity.

Fix the $a, b, c, \kappa_{0}, \kappa_{1}(\cdot), \kappa_{2}(\cdot)$ of the previous subsection, and the corresponding constants $\alpha_{0}^{i}, \alpha_{1}-\alpha_{5}$ for some trader $i$. We fix some admissible demand process $D$, and size-discovery reports $\mu$, by which the inventory of trader $i$ at time $t$, including the jumps associated with size-discovery sessions, is

$$
\hat{z}_{t}^{(D, \mu)}=z_{0}^{i}+\int_{0}^{t} D_{s}^{i} d s+H_{t}^{i}+\int_{0}^{t} Y^{i}\left(\left(\hat{\mu}_{s}, z_{s}^{-i}\right)\right) d N_{s},
$$

where $\hat{\mu}$ is the optional projection of the report process. 
We define

$$
U_{t}=1_{t<\mathcal{T}} V\left(\hat{z}_{t}^{D, \mu}, Z_{t}\right)+1_{t \geq \mathcal{T}} v \hat{z}_{t}^{D, \mu} .
$$

Following the steps of the derivation of the value function, we can show that under the laws of motion implied by $D$ and $\mu$,

$$
\mathbb{E}\left(U_{\mathcal{T}}-U_{0}\right)=\mathbb{E}\left(\int_{0}^{\mathcal{T}} \hat{\zeta}_{s} d s\right)
$$

where

$$
\begin{aligned}
\hat{\zeta}_{s}= & D_{s}\left(\alpha_{1}+\alpha_{5} \bar{Z}_{s}+2 \alpha_{3} \hat{z}_{s}^{D, \mu}\right)+\alpha_{4} \frac{\sigma_{Z}^{2}}{n^{2}}+\alpha_{3} \sigma_{i}^{2}+\alpha_{5} \frac{\rho^{i}}{n} \\
& +\lambda Y^{i}\left(\left(\hat{\mu}_{s}, z_{s}^{-i}\right)\right)\left(\alpha_{1}+\alpha_{5} \bar{Z}_{s}+2 \alpha_{3} \hat{z}_{s}^{D, \mu}+\alpha_{3} Y^{i}\left(\left(\hat{\mu}_{s}, z_{s}^{-i}\right)\right)\right)+r\left(v \hat{z}_{s}^{D, \mu}-V\left(\hat{z}_{s}^{D, \mu}, Z_{s}\right)\right) .
\end{aligned}
$$

Since $\alpha_{0}$ through $\alpha_{5}$ satisfy the HJB equation,

$$
\mathbb{E}\left[U_{\mathcal{T}}-U_{0}\right] \leq \mathbb{E}\left[\int_{0}^{\mathcal{T}} D_{s} \Phi_{(a, b, c)}\left(D_{s} ; Z_{s}-\hat{z}_{s}^{D, \mu}\right)+\gamma\left(\hat{z}_{s}^{D, \mu}\right)^{2} d s-\int_{0}^{\mathcal{T}} T_{\kappa}^{i}\left(\left(\hat{\mu}_{s}, z_{s}^{-i}\right), Z_{s}\right) d N_{s}\right] .
$$

Rearranging,

$V\left(z_{0}^{i}, Z_{0}\right) \geq \mathbb{E}\left[\pi \hat{z}_{\mathcal{T}}^{D, \mu}-\int_{0}^{\mathcal{T}} D_{s} \Phi_{(a, b, c)}\left(D_{s} ; Z_{s}-\hat{z}_{s}^{D, \mu}\right)+\gamma\left(\hat{z}_{s}^{D, \mu}\right)^{2} d s+\sum_{\left\{k: \tau_{k}<\mathcal{T}\right\}} T_{\kappa}^{i}\left(\left(\mu_{k}, z_{\tau_{k}}^{-i}\right), Z_{\tau_{k}}\right)\right]$.

Because this relationship holds with equality for the conjectured affine strategy and truthful size-discovery reporting, this candidate equilibrium strategy is optimal.

\section{E Numerical illustration}

Figure 1 illustrates the implications of augmenting the exchange market with size discovery. The figure shows simulated excess-inventory sample paths of two of the $n=10$ traders, with and without size discovery. The graphs of the asset positions shown in heavy line weights correspond to the market with size discovery. Those paths shown in light line weights correspond to the market with no size-discovery sessions (that is, with $\lambda=0$ ). In the market augmented with size discovery, the first such session is held at about time $t=10$, and causes a dramatic reduction in excess-inventory imbalances, bringing the excess inventories of all traders to the perfectly efficient level, the cross-sectional average excess inventory $\bar{Z}\left(\tau_{1}\right)=-0.05$. However, because traders shade their exchange bids more with the prospect of upcoming size-discovery sessions, from roughly time 110 until time 680, the market without size discovery performed dramatically better, ex post, than the market with size discovery. 


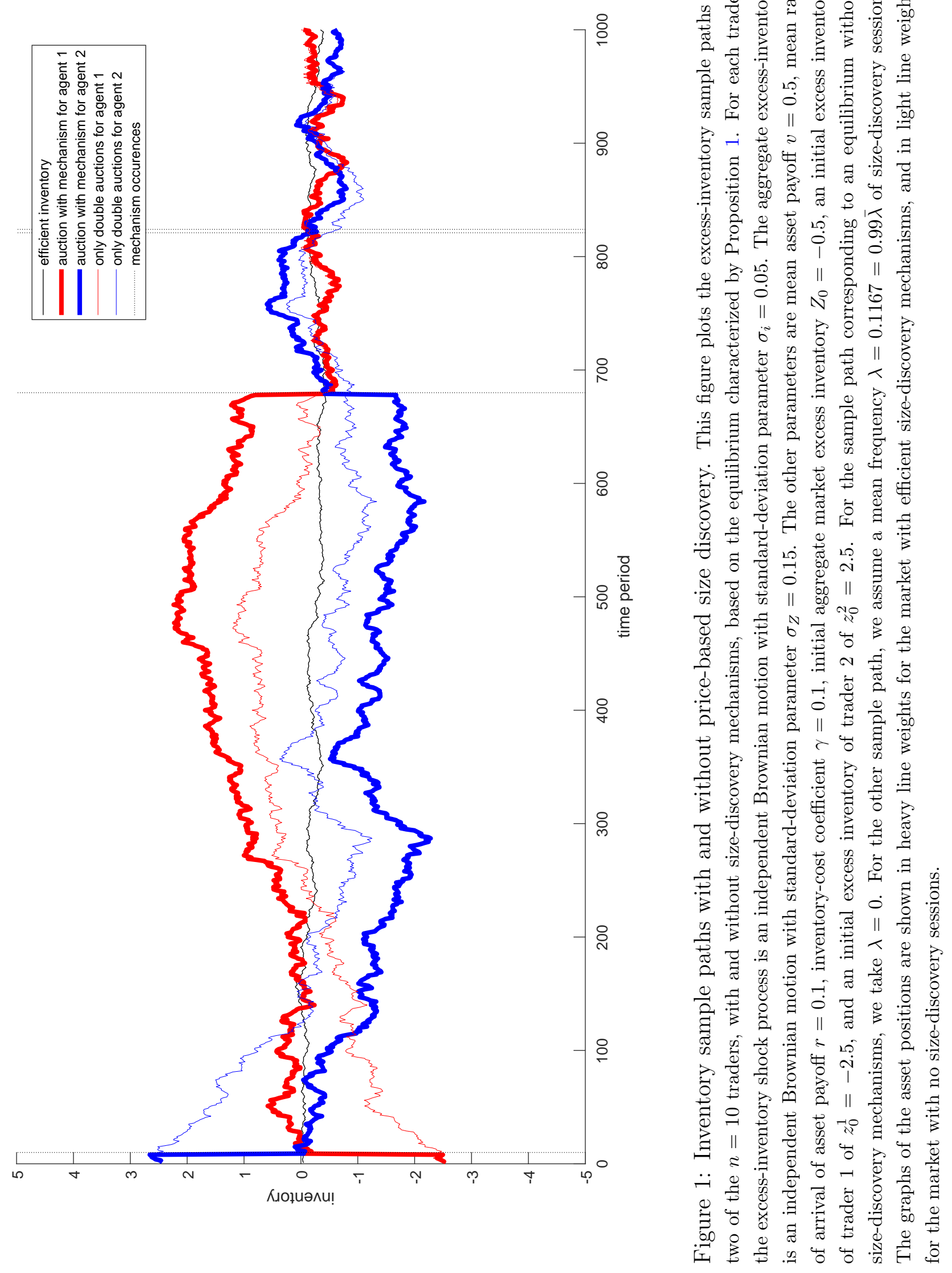




\section{F Discrete-time results}

In this appendix, we analyze discrete-time versions of the model. The focus is the existence of a subgame perfect equilibrium in each complete-information game, which corresponds to a Perfect Bayes equilibrium of each incomplete-information game. We also show convergence results for the main model of Section 3 and for the model found in Appendix D. All these results are presented informally, with focus on the calculation of the equilibrium, but these arguments can all be made fully rigorous.

The primitive setting, other than mechanisms, is identical to Duffie and Zhu (2017). Specifically, $n>2$ traders trade in each period $k \in\{0,1,2, \ldots\}$, where trading periods are separated by clock time $\Delta$ so that the $k$-th auction occurs at time $k \Delta$.

In each period $k$, each trader $i$ submits an auction order $x_{i k}\left(p_{k}\right)$ for how many units of asset they wish to purchase if the auction price is $p_{k}$. We focus on affine equilibria in which each trader chooses

$$
x_{i k}\left(p_{k}\right)=a+b p_{k}+c z_{i k},
$$

where $z_{i k}$ is the excess inventory of trader $i$ when entering period $k$, for some constants $a, c$ and $b \neq 0$. If $n-1$ traders use such a strategy with the same constants $a, b, c$, then there is a unique market-clearing price $\Phi_{(a, b, c)}(D, Z-z)$ for any demand $D$ submitted by trader $i$, which is given by

$$
\Phi_{(a, b, c)}(D, Z-z)=\frac{(n-1) a+c\left(Z_{k}-z_{i k}\right)+D}{-b(n-1)} .
$$

Each trader also submits a contingent mechanism report $\hat{z}_{i k}\left(p_{k}\right)$. With probability $q$, a mechanism occurs, and in that event trader $i$ receives a net reallocation

$$
Y^{i}(\hat{z})=\frac{\sum_{j=1}^{n} \hat{z}_{j k}}{n}-\hat{z}_{i k}
$$

and a cash transfer that will be described shortly, and that might depend upon $p_{k}$. With probability $1-q$, a double auction occurs, and each trader receives $x_{i k}\left(p_{k}\right)$ units of the asset at a cost $p_{k} x_{i k}\left(p_{k}\right)$. If trader $i$ ends period $k$ with inventory $z_{i k}^{+}$, then in between periods $k$ and $k+1$, they receive flow expected utility

$$
-\frac{\gamma}{r}\left(1-e^{-r \Delta}\right)\left(z_{i k}^{+}\right)^{2}+v\left(1-e^{-r \Delta}\right)\left(z_{i k}^{+}\right)
$$

which can be motivated as in Duffie and Zhu (2017). Let $\mathbf{1}_{M^{k}}$ equal 1 if and only if a mechanism occurs in period $k$, and let $\mathbf{1}_{M^{k}}^{c}=1-\mathbf{1}_{M^{k}}$. Then, in any equilibrium in which mechanisms implement efficient allocations, the equilibrium inventory evolves as

$$
z_{i, k+1}=w_{i, k+1}+\mathbf{1}_{M^{k}} \bar{Z}_{k}+\mathbf{1}_{M^{k}}^{c}\left((1+c) z_{i, k}-c \bar{Z}_{k}\right)
$$

where $\left\{w_{i, k+1}\right\}$ is a sequence of $i . i . d$ zero-mean finite-variance random variables. 


\section{F.1 Observable $Z_{t}$}

Suppose the aggregate inventory $Z_{k}$ is observable, and that transfers are given by

$$
T_{\kappa}^{i}(\hat{z}, Z)=\kappa_{0}\left(n \kappa_{2}\left(Z_{k}\right)+\sum_{j=1}^{n} \hat{z}_{j k}\right)^{2}+\kappa_{1}\left(Z_{k}\right)\left(\hat{z}_{i k}+\kappa_{2}\left(Z_{k}\right)\right)+\frac{\kappa_{1}\left(Z_{k}\right)^{2}}{4 \kappa_{0} n^{2}} .
$$

Just as in the continuous-time proof, at the equilibrium reports for affine $\kappa_{1}(\cdot), \kappa_{2}(\cdot)$, this must take the form

$$
R_{0}+R_{1} Z_{k}+R_{2} Z_{k}^{2}+R_{3} Z_{k} z_{i k}+R_{4} z_{i k}
$$

We solve for a subgame perfect equilibrium in which trader $i$ submits the demand

$$
x_{i k}\left(p_{k}\right)=a+b p_{k}+c z_{i k}
$$

and the report

$$
\hat{z}_{i k}\left(p_{k}\right)=z_{i k}
$$

In such an equilibrium, the continuation value $V(z, Z)$ must be linear quadratic. Specifically, the continuation value is

$$
V(z, Z)=\mathbb{E}\left[\sum_{k=0}^{\infty} e^{-r \Delta k} \tilde{\pi}_{k}\right]
$$

where

$$
\begin{aligned}
\tilde{\pi}_{k}=q & \left(R_{0}+R_{1} Z_{k}+R_{2} Z_{k}^{2}+R_{3} Z_{k} z_{i k}+R_{4} z_{i k}-\frac{\gamma}{r}\left(1-e^{-r \Delta}\right)\left(\bar{Z}_{k}\right)^{2}+v\left(1-e^{-r \Delta}\right)\left(\bar{Z}_{k}\right)\right) \\
& +(1-q)\left(-x_{i k}\left(p_{k}\right) p_{k}-\frac{\gamma}{r}\left(1-e^{-r \Delta}\right)\left(x_{i k}\left(p_{k}\right)+z_{i k}\right)^{2}+v\left(1-e^{-r \Delta}\right)\left(x_{i k}\left(p_{k}\right)+z_{i k}\right)\right) .
\end{aligned}
$$

We are given that $z_{i 0}=z, Z_{0}=Z, \sum_{i=1}^{n} x_{i k}\left(p_{k}\right)=0$, and

$$
z_{i, k+1}=w_{i, k+1}+\mathbf{1}_{M^{k}}\left(z_{i k}+\frac{\sum_{j=1}^{n} \hat{z}_{j k}}{n}-\hat{z}_{i k}\right)+\mathbf{1}_{M^{k}}^{c}\left(z_{i k}+x_{i k}\left(p_{k}\right)\right) .
$$

Fix the conjectured equilibrium $a, b, c$ with truth-telling $\left(\hat{z}_{i k}=z_{i k}\right)$, so that

$$
z_{i, k+1}=w_{i, k+1}+\mathbf{1}_{M^{k}} \bar{Z}_{k}+\mathbf{1}_{M^{k}}^{c}\left((1+c) z_{i, k}-c \bar{Z}_{k}\right)
$$

The expression for $V(z, Z)$ can be decomposed into a linear combination of discounted sums 
of moments of $z_{i k}, Z_{k}$. We calculate these now. Straightforward calculation shows that

$$
\begin{aligned}
& \mathbb{E}\left[\sum_{k=0}^{\infty} e^{-r \Delta k} Z_{k}\right]=\frac{Z_{0}}{1-e^{-r \Delta}}=S_{0} Z_{0} \\
& \mathbb{E}\left[\sum_{k=0}^{\infty} e^{-r \Delta k} Z_{k}^{2}\right]=\frac{Z_{0}^{2}}{1-e^{-r \Delta}}+\frac{\sigma_{Z}^{2} e^{-r \Delta}}{1-e^{-r \Delta}}=S_{0} Z_{0}^{2}+S_{1}
\end{aligned}
$$

where $\sigma_{Z}^{2} \equiv \operatorname{var}\left(\sum_{i=1}^{n} w_{i, k+1}\right)$. Subtracting $\bar{Z}_{i, k+1}$ from both sides of equation (96), rearranging, and taking an expectation gives

$$
\mathbb{E}\left[z_{i, k+1}-\bar{Z}_{k+1}\right]=(1-q)(1+c) \mathbb{E}\left[z_{i, k}-\bar{Z}_{k}\right] .
$$

Some calculations then show that

$$
\mathbb{E}\left[\sum_{k=0}^{\infty} e^{-r \Delta k} z_{i k}\right]=\frac{z_{i 0}-\bar{Z}_{0}}{1-e^{-r \Delta}(1+c)(1-q)}+\frac{\bar{Z}_{0}}{1-e^{-r \Delta}}=S_{2}\left(z_{i 0}-\bar{Z}_{0}\right)+S_{0} \bar{Z}_{0},
$$

provided that $\left|e^{-r \Delta}(1+c)(1-q)\right|<1$. Subtracting $\bar{Z}_{i, k+1}$ from both sides of equation (96), then multiplying both sides by $\bar{Z}_{i, k+1}$, and taking an expectation gives

$$
E\left[z_{i, k+1} Z_{k+1}-\bar{Z}_{k+1}^{2}\right]=\left(\frac{\rho^{i}}{n}-\frac{\sigma_{Z}^{2}}{n^{2}}\right)+(1-q)(1+c) E\left[z_{i, k} \bar{Z}_{k}-\bar{Z}_{k}^{2}\right],
$$

where $\rho^{i}=\mathbb{E}\left[w_{i, k+1}\left(\sum_{i=1}^{n} w_{i, k+1}\right)\right]$.

Then we see that

$$
\begin{aligned}
& \mathbb{E}\left[\sum_{k=0}^{\infty} e^{-r \Delta k} z_{i k} \bar{Z}_{k}\right]=\mathbb{E}\left[\sum_{k=0}^{\infty} e^{-r \Delta k}\left(z_{i k} \bar{Z}_{k}-\bar{Z}_{k}^{2}\right)\right]+S_{0} \bar{Z}_{0}^{2}+\frac{S_{1}}{n^{2}} \\
& =z_{i 0} \bar{Z}_{0}-\bar{Z}_{0}^{2}+e^{-r \Delta} \sum_{k=1}^{\infty} e^{-r \Delta(k-1)} \mathbb{E}\left[z_{i k} \bar{Z}_{k}-\bar{Z}_{k}^{2}\right]+S_{0} \bar{Z}_{0}^{2}+\frac{S_{1}}{n^{2}} \\
& =z_{i 0} \bar{Z}_{0}-\bar{Z}_{0}^{2}+S_{0} \bar{Z}_{0}^{2}+\frac{S_{1}}{n^{2}} \\
& +e^{-r \Delta} \mathbb{E}\left[\sum_{k=0}^{\infty} e^{-r \Delta k}\left(\left(\frac{\rho^{i}}{n}-\frac{\sigma_{Z}^{2}}{n^{2}}\right)+(1-q)(1+c) E\left[z_{i, k} \bar{Z}_{k}-\bar{Z}_{k}^{2}\right]\right)\right] \\
& =z_{i 0} \bar{Z}_{0}-\bar{Z}_{0}^{2}+\frac{e^{-r \Delta}\left(\frac{\rho^{i}}{n}-\frac{\sigma_{Z}^{2}}{n^{2}}\right)}{1-e^{-r \Delta}}+\left(1-e^{-r \Delta}(1-q)(1+c)\right)\left(S_{0} \bar{Z}_{0}^{2}+\frac{S_{1}}{n^{2}}\right) \\
& +(1-q)(1+c) e^{-r \Delta} \mathbb{E}\left[\sum_{k=0}^{\infty} e^{-r \Delta k} z_{i k} \bar{Z}_{k}\right] .
\end{aligned}
$$


Rearranging delivers

$$
\begin{aligned}
\mathbb{E}\left[\sum_{k=0}^{\infty} e^{-r \Delta k} z_{i k} \bar{Z}_{k}\right] & =S_{0} \bar{Z}_{0}^{2}+\frac{S_{1}}{n^{2}}+\frac{z_{i 0} \bar{Z}_{0}-\bar{Z}_{0}^{2}+\frac{e^{-r \Delta}\left(\frac{\rho^{i}}{n}-\frac{\sigma_{Z}^{2}}{n^{2}}\right)}{1-e^{-r \Delta}}}{1-(1-q)(1+c) e^{-r \Delta}} \\
& =S_{2} z_{i 0} \bar{Z}_{0}+\left(S_{0}-S_{2}\right) \bar{Z}_{0}^{2}+S_{3} .
\end{aligned}
$$

Finally, squaring both sides of equation (96) and taking an expectation shows that

$$
\mathbb{E}\left[\left(z_{i, k+1}-\bar{Z}_{k+1}\right)^{2}\right]=\left(\frac{\sigma_{Z}^{2}}{n^{2}}-2 \frac{\rho^{i}}{n}+\sigma_{i}^{2}\right)+(1-q)(1+c)^{2} \mathbb{E}\left[\left(z_{i, k}-\bar{Z}_{k}\right)^{2}\right],
$$

where $\sigma_{i}^{2}=\mathbb{E}\left[w_{i, k+1}^{2}\right]$.

Then,

$$
\sum_{k=0}^{\infty} e^{-r \Delta} \mathbb{E}\left[\left(z_{i, k}-\bar{Z}_{k}\right)^{2}\right]=\frac{\left(z_{i, 0}-\bar{Z}_{0}\right)^{2}+\frac{\left(\frac{\sigma_{Z}^{2}}{n^{2}}-2 \frac{\rho^{i}}{n}+\sigma_{i}^{2}\right) e^{-r \Delta}}{1-e^{-r \Delta}}}{1-e^{-r \Delta}(1-q)(1+c)^{2}}=S_{4}\left(z_{i, 0}-\bar{Z}_{0}\right)^{2}+S_{5}
$$

provided that $\left|S_{4}^{-1}\right|=\left|1-e^{-r \Delta}(1-q)(1+c)^{2}\right|<1$. It follows that

$$
\sum_{k=0}^{\infty} e^{-r \Delta} \mathbb{E}\left[z_{i, k}^{2}\right]=S_{4}\left(z_{i, 0}-\bar{Z}_{0}\right)^{2}+S_{5}+2\left(S_{2} z_{i 0} \bar{Z}_{0}+\left(S_{0}-S_{2}\right) \bar{Z}_{0}^{2}+S_{3}\right)-\left(S_{0} \bar{Z}_{0}^{2}+\frac{S_{1}}{n^{2}}\right) .
$$

In summary, letting

$$
\begin{aligned}
& S_{0}=\frac{1}{1-e^{-r \Delta}} \\
& S_{1}=\frac{\sigma_{Z}^{2} e^{-r \Delta}}{1-e^{-r \Delta}} \\
& S_{2}=\frac{1}{1-e^{-r \Delta}(1-q)(1+c)} \\
& S_{3}=S_{2} \frac{e^{-r \Delta}\left(\frac{\rho^{i}}{n}-\frac{\sigma_{Z}^{2}}{n^{2}}\right)}{1-e^{-r \Delta}} \\
& S_{4}=\frac{1}{1-e^{-r \Delta}(1-q)(1+c)^{2}} \\
& S_{5}=S_{4} \frac{\left(\frac{\sigma_{Z}^{2}}{n^{2}}-2 \frac{\rho^{i}}{n}+\sigma_{i}^{2}\right) e^{-r \Delta}}{1-e^{-r \Delta}}
\end{aligned}
$$


and assuming $\left|S_{2}^{-1}\right|,\left|S_{4}^{-1}\right|$ are strictly less than 1 , we have

$$
\begin{aligned}
& \mathbb{E}\left[\sum_{k=0}^{\infty} e^{-r \Delta k} z_{i k}\right]=S_{2}\left(z_{i 0}-\bar{Z}_{0}\right)+S_{0} \bar{Z}_{0} \\
\mathbb{E}\left[\sum_{k=0}^{\infty} e^{-r \Delta k} z_{i k} \bar{Z}_{k}\right] & =S_{2} z_{i 0} \bar{Z}_{0}+\left(S_{0}-S_{2}\right) \bar{Z}_{0}^{2}+S_{3} \\
& \mathbb{E}\left[\sum_{k=0}^{\infty} e^{-r \Delta k} \bar{Z}_{k}\right]=S_{0} \bar{Z}_{0} \\
& \mathbb{E}\left[\sum_{k=0}^{\infty} e^{-r \Delta k} \bar{Z}_{k}^{2}\right]=S_{0} \bar{Z}_{0}^{2}+\frac{S_{1}}{n^{2}} \\
& \mathbb{E}\left[\sum_{k=0}^{\infty} e^{-r \Delta} z_{i, k}^{2}\right]=S_{4}\left(z_{i, 0}-\bar{Z}_{0}\right)^{2}+S_{5}+2\left(S_{2} z_{i 0} \bar{Z}_{0}+\left(S_{0}-S_{2}\right) \bar{Z}_{0}^{2}+S_{3}\right)-\left(S_{0} \bar{Z}_{0}^{2}+\frac{S_{1}}{n^{2}}\right) .
\end{aligned}
$$

Suppose that

$$
V(z, Z)=\alpha_{0}^{i}+\alpha_{1} z+\alpha_{2} \bar{Z}+\alpha_{3} z^{2}+\alpha_{4} \bar{Z}^{2}+\alpha_{5} z \bar{Z}
$$

Then the utility for inventory $(z, Z)$ immediately after an auction or mechanism is

$$
\begin{aligned}
V^{+}(z, Z)=- & \frac{\gamma}{r}\left(1-e^{-r \Delta}\right)(z)^{2}+v\left(1-e^{-r \Delta}\right) z+\mathbb{E}\left[e^{-r \Delta} V\left(z+w_{i, k+1}, Z+\sum_{i} w_{i, k+1}\right)\right] \\
=- & \frac{\gamma}{r}\left(1-e^{-r \Delta}\right)(z)^{2}+v\left(1-e^{-r \Delta}\right) z \\
& \quad+e^{-r \Delta}\left(\alpha_{0}^{i}+\alpha_{3} \sigma_{i}^{2}+\alpha_{4} \frac{\sigma_{Z}^{2}}{n^{2}}+\alpha_{5} \frac{\rho^{i}}{n}+\alpha_{1} z+\alpha_{2} \bar{Z}+\alpha_{3} z^{2}+\alpha_{4} \bar{Z}^{2}+\alpha_{5} z \bar{Z}\right) \\
= & u(Z)+\left(e^{-r \Delta} \alpha_{3}-\frac{\gamma}{r}\left(1-e^{-r \Delta}\right)\right)(z-\bar{Z})^{2}+\left(v\left(1-e^{-r \Delta}\right)+e^{-r \Delta} \alpha_{1}\right) z \\
& \quad+\left(e^{-r \Delta} \alpha_{5}+2\left(e^{-r \Delta} \alpha_{3}-\frac{\gamma}{r}\left(1-e^{-r \Delta}\right)\right)\right) z \bar{Z} .
\end{aligned}
$$

We have thus shown that the continuation value maximized in the mechanism takes the form found in Appendix A, with

$$
\begin{aligned}
& \beta_{0}=\left(v\left(1-e^{-r \Delta}\right)+e^{-r \Delta} \alpha_{1}\right) \\
& \beta_{1}=e^{-r \Delta} \alpha_{5}+2\left(e^{-r \Delta} \alpha_{3}-\frac{\gamma}{r}\left(1-e^{-r \Delta}\right)\right) .
\end{aligned}
$$

To meet the individual-rationality restriction, transfers in the mechanism thus must be run with $\kappa_{1}\left(Z_{k}\right)=\beta_{0}+\beta_{1} \bar{Z}_{k}$. From Proposition 3 , in the equilibrium of the mechanism game that we seek (with observable $Z$ ), each trader submits $\hat{z}_{i k}=z_{i k}$ as long as

$$
\kappa_{2}\left(Z_{k}\right)=-\bar{Z}_{k}+\frac{-\left(\beta_{0}+\beta_{1} \bar{Z}_{k}\right)}{2 \kappa_{0} n^{2}},
$$


so that

$$
n \kappa_{2}\left(Z_{k}\right)+\sum_{i} \hat{z}_{i k}=\frac{-\left(\beta_{0}+\beta_{1} \bar{Z}_{k}\right)}{2 \kappa_{0} n} .
$$

Returning to the continuation value, in equilibrium at each mechanism event, trader $i$ receives the cash transfer $\kappa_{1}\left(Z_{k}\right)\left(z_{i k}-\bar{Z}\right)=\left(\beta_{0}+\beta_{1} \bar{Z}_{k}\right)\left(z_{i k}-\bar{Z}\right)$. The equilibrium price must be $p_{k}=-(a+c \bar{Z}) / b$ and the equilibrium double-auction demand is $x_{i k}=c\left(z_{i k}-\bar{Z}_{k}\right)$. Thus, plugging in, the candidate equilibrium continuation value is

$$
V(z, Z)=\mathbb{E}\left[\sum_{k=0}^{\infty} e^{-r \Delta k} \hat{\pi}_{k}\right]
$$

where

$$
\begin{aligned}
\hat{\pi}_{k}=q & \left(\left(\beta_{0}+\beta_{1} \bar{Z}_{k}\right)\left(z_{i k}-\bar{Z}_{k}\right)-\frac{\gamma}{r}\left(1-e^{-r \Delta}\right)\left(\bar{Z}_{k}\right)^{2}+v\left(1-e^{-r \Delta}\right)\left(\bar{Z}_{k}\right)\right) \\
& +(1-q)\left(-c\left(z_{i k}-\bar{Z}_{k}\right) \frac{a+c \bar{Z}_{k}}{-b}-\frac{\gamma}{r}\left(1-e^{-r \Delta}\right)\left((1+c) z_{i k}-c \bar{Z}_{k}\right)^{2}\right) \\
& +(1-q)\left(v\left(1-e^{-r \Delta}\right)\left((1+c) z_{i k}-c \bar{Z}_{k}\right)\right) .
\end{aligned}
$$

Collecting terms,

$$
\begin{aligned}
V(z, Z) & =\left(q \beta_{0}+(1-q)\left[\frac{c a}{b}+v\left(1-e^{-r \Delta}\right)(1+c)\right]\right) \mathbb{E}\left[\sum_{k=0}^{\infty} e^{-r \Delta k} z_{i k}\right] \\
& +\left(q \beta_{1}+(1-q)\left[\frac{c^{2}}{b}+2 \frac{\gamma}{r}\left(1-e^{-r \Delta}\right)(1+c) c\right]\right) \mathbb{E}\left[\sum_{k=0}^{\infty} e^{-r \Delta k} z_{i k} \bar{Z}_{k}\right] \\
& -\frac{\gamma}{r}\left(1-e^{-r \Delta}\right)(1-q)(1+c)^{2} \mathbb{E}\left[\sum_{k=0}^{\infty} e^{-r \Delta k} z_{i k}^{2}\right]+\epsilon(Z) .
\end{aligned}
$$

Plugging in the definitions found above, it follows that

$$
\begin{aligned}
& \alpha_{1}=S_{2}\left(q \beta_{0}+(1-q)\left[\frac{c a}{b}+v\left(1-e^{-r \Delta}\right)(1+c)\right]\right) \\
& \alpha_{3}=-\frac{\gamma}{r}\left(1-e^{-r \Delta}\right)(1-q)(1+c)^{2} S_{4} \\
& \alpha_{5}=S_{2}\left(q \beta_{1}+(1-q)\left[\frac{c^{2}}{b}+2 \frac{\gamma}{r}\left(1-e^{-r \Delta}\right)(1+c) c\right]\right)-\frac{\gamma}{r}\left(1-e^{-r \Delta}\right)(1-q)(1+c)^{2}\left(2\left(S_{2}-S_{4}\right)\right) .
\end{aligned}
$$


Recalling the expressions for $\beta_{0}, S_{2}$, the formula for $\alpha_{1}$ implies that

$$
\begin{aligned}
\beta_{0} & =v\left(1-e^{-r \Delta}\right)+e^{-r \Delta} \alpha_{1} \\
& =v\left(1-e^{-r \Delta}\right)+\frac{e^{-r \Delta}}{1-e^{-r \Delta}(1-q)(1+c)}\left(q \beta_{0}+(1-q)\left[\frac{c a}{b}+v\left(1-e^{-r \Delta}\right)(1+c)\right]\right) .
\end{aligned}
$$

So, conjecturing and later verifying that $1-e^{-r \Delta}(1-q)(1+c)-q e^{-r \Delta} \neq 0$, we have

$$
\beta_{0}=\left(\frac{1-e^{-r \Delta}(1-q)(1+c)}{1-e^{-r \Delta}(1-q)(1+c)-q e^{-r \Delta}}\right) \tau_{c}
$$

where

$$
\tau_{c}=v\left(1-e^{-r \Delta}\right)+\frac{e^{-r \Delta}(1-q)}{1-e^{-r \Delta}(1-q)(1+c)}\left[\frac{c a}{b}+v\left(1-e^{-r \Delta}\right)(1+c)\right] .
$$

A similar calculation shows that

$$
\begin{aligned}
\beta_{1}=e^{-r \Delta} S_{2} q \beta_{1}+e^{-r \Delta} S_{2}\left((1-q)\left[\frac{c^{2}}{b}+2 \frac{\gamma}{r}\left(1-e^{-r \Delta}\right)(1+c) c\right]\right) \\
\quad-\frac{e^{-r \Delta} \gamma}{r}\left(1-e^{-r \Delta}\right)(1-q)(1+c)^{2}\left(2\left(S_{2}-S_{4}\right)\right)+2\left(e^{-r \Delta} \alpha_{3}-\frac{\gamma}{r}\left(1-e^{-r \Delta}\right)\right) .
\end{aligned}
$$

and thus

$$
\beta_{1}=\zeta_{a}\left(\tau_{d}+\tau_{e}\right)
$$

where

$$
\begin{aligned}
\zeta_{a} & =\frac{1-e^{-r \Delta}(1-q)(1+c)}{1-e^{-r \Delta}(1-q)(1+c)-q e^{-r \Delta}} \\
\tau_{d} & =e^{-r \Delta} S_{2}(1-q)\left[\frac{c^{2}}{b}+2 \frac{\gamma}{r}\left(1-e^{-r \Delta}\right)(1+c) c\right] \\
\tau_{e} & =-\frac{e^{-r \Delta} \gamma}{r}\left(1-e^{-r \Delta}\right)(1-q)(1+c)^{2}\left(2\left(S_{2}-S_{4}\right)\right)+2\left(e^{-r \Delta} \alpha_{3}-\frac{\gamma}{r}\left(1-e^{-r \Delta}\right)\right) .
\end{aligned}
$$

Putting this all together, the continuation value for trader $i$ in a symmetric equilibrium, immediately after an auction or mechanism is run, is

$$
V^{+}(z, Z)=u(Z)-\frac{\gamma}{r}\left(1-e^{-r \Delta}\right)\left[(1-q)(1+c)^{2} S_{4} e^{-r \Delta}+1\right](z-\bar{Z})^{2}+\left(\beta_{0}+\beta_{1} \bar{Z}\right)(z-\bar{Z}) .
$$

Plugging in the definition of $S_{4}$, this simplifies slightly to

$$
V^{+}(z, Z)=u(Z)+\frac{-\frac{\gamma}{r}\left(1-e^{-r \Delta}\right)}{1-e^{-r \Delta}(1-q)(1+c)^{2}}(z-\bar{Z})^{2}+\left(\beta_{0}+\beta_{1} \bar{Z}\right)(z-\bar{Z}) .
$$


Trader $i$ can choose any quantity $x$ to purchase at the price

$$
\Phi(x)=\frac{1}{-b(n-1)}((n-1) a+c(Z-z)+x) .
$$

With observable $Z$, the order size $x$ is irrelevant to the payoff and continuation value in the event of a mechanism. Thus a trader with pre-trade position $z$ maximizes

$$
-x \frac{1}{-b(n-1)}((n-1) a+c(Z-z)+x)+V^{+}(z+x, Z)
$$

Differentiating this expression with respect to $x$ leaves

$$
-\Phi(x)+\frac{x}{b(n-1)}+\left(\beta_{0}+\beta_{1} \bar{Z}\right)-\frac{\frac{2 \gamma}{r}\left(1-e^{-r \Delta}\right)}{1-e^{-r \Delta}(1-q)(1+c)^{2}}(z+x-\bar{Z}),
$$

which must be 0 with $\Phi=\phi, \bar{Z}=(-a-b \phi) / c$, and $x=a+b \phi+c z$. The second-order condition is met if and only if $b<0$. This also implies $x=c(z-\bar{Z})$, so

$$
(z+x-\bar{Z})=(1+c) z+(1+c) \frac{a+b \phi}{c} .
$$

Plugging this in and gathering coefficients on $\phi, z, 1$, we have

$$
\begin{aligned}
& 0=-1+\frac{1}{n-1}-\frac{b \beta_{1}}{c}-\frac{\frac{2 \gamma}{r}\left(1-e^{-r \Delta}\right)}{1-e^{-r \Delta}(1-q)(1+c)^{2}}(1+c) \frac{b}{c} \\
& 0=\frac{c}{b(n-1)}-\frac{\frac{2 \gamma}{r}\left(1-e^{-r \Delta}\right)}{1-e^{-r \Delta}(1-q)(1+c)^{2}}(1+c) \\
& 0=\frac{a}{b(n-1)}+\left(\beta_{0}-\frac{a}{c} \beta_{1}\right)-\frac{\frac{2 \gamma}{r}\left(1-e^{-r \Delta}\right)}{1-e^{-r \Delta}(1-q)(1+c)^{2}}(1+c) \frac{a}{c} .
\end{aligned}
$$

We seek $a, b, c, \beta_{1}, \beta_{0}$ such that these three equations and the two equations defining $\beta_{0}, \beta_{1}$ all hold. Let $\omega$ be the larger root of

$$
e^{-r \Delta} \omega^{2}+(n-1)\left(1-e^{-r \Delta}\right) \omega-1=0
$$

so

$$
\omega=\frac{-(n-1)\left(1-e^{-r \Delta}\right)+\sqrt{(n-1)^{2}\left(1-e^{-r \Delta}\right)^{2}+4 e^{-r \Delta}}}{2 e^{-r \Delta}} .
$$

Then, in Duffie and Zhu (2017), where $q=0$, we can set

$$
a=\frac{r v}{2 \gamma}(1-\omega), \quad b=-\frac{r}{2 \gamma}(1-\omega), \quad c=-(1-\omega),
$$

and see that

$$
\frac{(1+c)\left(1-e^{-r \Delta}\right)}{1-e^{-r \Delta}(1+c)^{2}}=\frac{\frac{1-e^{-r \Delta} \omega^{2}}{n-1}}{1-e^{-r \Delta} \omega^{2}}=\frac{1}{n-1}
$$


It follows that the above system holds with $\beta_{0}=v, \beta_{1}=-2 \gamma / r$. Now, let $\hat{\omega}$ be the larger root of

$$
e^{-r \Delta}(1-q) \hat{\omega}^{2}+(n-1)\left(1-e^{-r \Delta}\right) \hat{\omega}-1=0,
$$

so that

$$
\hat{\omega}=\frac{-(n-1)\left(1-e^{-r \Delta}\right)+\sqrt{(n-1)^{2}\left(1-e^{-r \Delta}\right)^{2}+4(1-q) e^{-r \Delta}}}{2(1-q) e^{-r \Delta}} .
$$

This implies that, letting $a, b, c$ be as before but replacing $\omega$ with $\hat{\omega}$,

$$
\frac{(1+c)\left(1-e^{-r \Delta}\right)}{1-e^{-r \Delta}(1-q)(1+c)^{2}}=\frac{\frac{1-e^{-r \Delta}(1-q) \hat{\omega}^{2}}{n-1}}{1-e^{-r \Delta}(1-q) \hat{\omega}^{2}}=\frac{1}{n-1} .
$$

It is straightforward to show that $a, b, c$ defined with $\hat{\omega}$, and $\beta_{0}=v, \beta_{1}=-2 \gamma / r$ once again solve the above system. We now must verify that they satisfy the definitions of $\beta_{0}, \beta_{1}$. Note that under the conjectured values,

$$
\begin{aligned}
q \beta_{0}+(1-q)\left[\frac{c a}{b}+v\left(1-e^{-r \Delta}\right)(1+c)\right] & =v\left(q+(1-q)\left[-(1+c)+1+\left(1-e^{-r \Delta}\right)(1+c)\right]\right) \\
& =v\left(1-e^{-r \Delta}(1+c)(1-q)\right),
\end{aligned}
$$

from which it can be seen that $\beta_{0}=v$ is consistent with the earlier system. We noted above that

$$
\left(-\frac{\gamma}{r}\left(1-e^{-r \Delta}\right)(1-q)(1+c)^{2} S_{4} e^{-r \Delta}-\frac{\gamma}{r}\left(1-e^{-r \Delta}\right)\right)=\frac{-\frac{\gamma}{r}\left(1-e^{-r \Delta}\right)}{1-e^{-r \Delta}(1-q)(1+c)^{2}} .
$$

Plugging this into the definition of $\beta_{1}$, we have

$$
\beta_{1}=\zeta_{a}\left(\tau_{d}+\tau_{f}\right),
$$

where $\zeta_{a}$ and $\tau_{d}$ are defined above and

$$
\tau_{f}=-\frac{e^{-r \Delta} \gamma}{r}\left(1-e^{-r \Delta}\right)(1-q)(1+c)^{2}\left(2\left(S_{2}-S_{4}\right)\right)+2 \frac{-\frac{\gamma}{r}\left(1-e^{-r \Delta}\right)}{1-e^{-r \Delta}(1-q)(1+c)^{2}} .
$$

Rearranging, we see that

$$
\begin{array}{r}
\frac{e^{-r \Delta} \gamma}{r}\left(1-e^{-r \Delta}\right)(1-q)(1+c)^{2}\left(2 S_{4}\right)+2 \frac{-\frac{\gamma}{r}\left(1-e^{-r \Delta}\right)}{1-e^{-r \Delta}(1-q)(1+c)^{2}} \\
=2\left(1-e^{-r \Delta}\right) \frac{\gamma}{r}\left[e^{-r \Delta}(1-q)(1+c)^{2} S_{4}-S_{4}\right],
\end{array}
$$

where $e^{-r \Delta}(1-q)(1+c)^{2} S_{4}-S_{4}=-1$.

Pulling together terms involving $S_{2}$ and noting $(1+c) c-(1+c)^{2}=-(1+c)$, we have

$$
\beta_{1}=\zeta_{a}\left[e^{-r \Delta} S_{2}\left((1-q)\left[\frac{c^{2}}{b}-2 \frac{\gamma}{r}\left(1-e^{-r \Delta}\right)(1+c)\right]\right)-2\left(1-e^{-r \Delta}\right) \frac{\gamma}{r}\right] .
$$


Multiplying and dividing the last term by $S_{2}$, we arrive at

$$
\beta_{1}=\zeta_{a}\left[e^{-r \Delta} S_{2}\left((1-q) \frac{c^{2}}{b}-2 \frac{\gamma}{r}\left(1-e^{-r \Delta}\right) e^{r \Delta}\right)\right]
$$

Applying the definition of $S_{2}$,

$$
\beta_{1}=\frac{e^{-r \Delta}\left((1-q) \frac{c^{2}}{b}-2 \frac{\gamma}{r}\left(1-e^{-r \Delta}\right) e^{r \Delta}\right)}{1-e^{-r \Delta}(1-q)(1+c)-q e^{-r \Delta}} .
$$

Finally, we can plug in the conjectured $a, b, c$, so that $c^{2} / b=(2 \gamma / r) c$, and rearrange to find

$$
\beta_{1}=-2 \frac{\gamma}{r} \frac{e^{-r \Delta}\left(-(1-q) c+\left(1-e^{-r \Delta}\right) e^{r \Delta}\right)}{1-e^{-r \Delta}(1-q)(1+c)-q e^{-r \Delta}}=-2 \frac{\gamma}{r}
$$

Thus the conjectured equilibrium is an equilibrium (filling in the implied $\alpha_{0}^{i}, \alpha_{2}, \alpha_{4}$ ). Finally, note that

$$
\frac{1-\hat{\omega}}{\Delta}=\frac{(n-1)\left(1-e^{-r \Delta}\right)+2(1-q) e^{-r \Delta}-\sqrt{(n-1)^{2}\left(1-e^{-r \Delta}\right)^{2}+4(1-q) e^{-r \Delta}}}{2(1-q) e^{-r \Delta} \Delta} .
$$

Suppose that $q=\lambda \Delta$, so this becomes

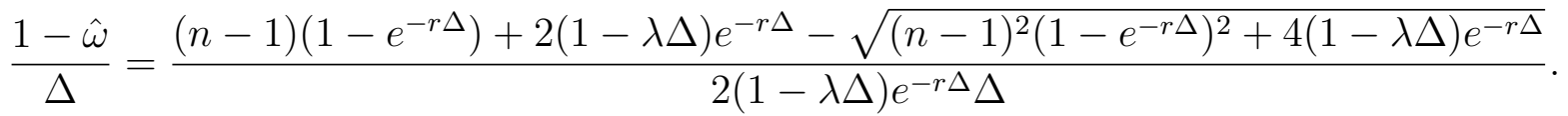

We multiply the denominator and numerator by $e^{r \Delta}$ and obtain

$$
\frac{1-\hat{\omega}}{\Delta}=\frac{(n-1)\left(e^{r \Delta}-1\right)+2(1-\lambda \Delta)-\sqrt{(n-1)^{2}\left(1-e^{r \Delta}\right)^{2}+4(1-\lambda \Delta) e^{r \Delta}}}{2(1-\lambda \Delta) \Delta} .
$$

The derivative of this expression with respect to $\Delta$ is

$$
\begin{aligned}
& {[2(1-2 \lambda \Delta)]^{-1}\left((n-1)\left(r e^{r \Delta}\right)-2 \lambda\right)} \\
& -\frac{\left((n-1)^{2}\left(1-e^{r \Delta}\right)^{2}+4(1-\lambda \Delta) e^{r \Delta}\right)^{-.5}\left(-2 r e^{r \Delta}(n-1)^{2}\left(1-e^{r \Delta}\right)+4 r(1-\lambda \Delta) e^{r \Delta}-4 \lambda e^{r \Delta}\right)}{4(1-2 \lambda \Delta)} .
\end{aligned}
$$

The limit of this expression as $\Delta \rightarrow 0$ is

$$
\frac{1}{2}((n-1) r-2 \lambda)-.5 \frac{(4)^{-.5}(4 r-4 \lambda)}{2}=\frac{(n-2) r-\lambda}{2} .
$$

By l'Hôpital's Rule,

$$
\lim _{\Delta \rightarrow 0} \frac{-(1-\hat{\omega})}{\Delta}=\frac{-(n-2) r+\lambda}{2}
$$


which is the instantaneous demand in the continuous-time model. It is immediate that $a, b$ converge to their corresponding limits, and since the strategies converge as $\Delta \rightarrow 0$, so too must the continuation values, for properly defined shocks.

\section{F.2 Unobservable $Z_{t}$}

Let the transfer function $T_{Q}^{i}$ be defined exactly as in the continuous-time model. As in the proof for the continuous-time model, in an equilibrium with truth-telling and affine $\delta$, cash transfers take the form

$$
R_{0}+R_{1} Z_{k}+R_{2} Z_{k}^{2}+R_{3} Z_{k} z_{i k}+R_{4} z_{i k}
$$

The value function is thus linear-quadratic, so, just as in the previous section, the equilibrium value function immediately after an auction or mechanism $V^{+}(z, Z)$ is linear quadratic in $(z, Z)$ and thus can be rewritten

$$
V^{+}(z, Z)=v_{0}+v_{1} z+v_{2} \bar{Z}+v_{3} z^{2}+v_{4} \bar{Z}^{2}+v_{5} z \bar{Z}
$$

for some constants $v_{0}, \ldots, v_{5}$. Then, following the steps of Appendix B.3, maximizing

$$
V^{+}\left(z+Y^{i}\left(\left(\hat{z}^{i}, \hat{z}^{-i}\right)\right), Z\right)+T_{Q}^{i}\left(\left(\hat{z}^{i}, \hat{z}^{-i}\right) ; p\right)
$$

is equivalent to maximizing

$$
\begin{aligned}
\mathcal{E}\left(p, Z, z^{i}, \hat{z}^{i}\right) \equiv( & \left.v_{1}+v_{5} \bar{Z}\right)\left(\frac{Z-z^{i}}{n}-\frac{n-1}{n} \hat{z}^{i}\right)+v_{3}\left(\frac{Z-z^{i}}{n}-\frac{n-1}{n} \hat{z}^{i}\right)^{2} \\
& +2 v_{3} z^{i}\left(\frac{Z-z^{i}}{n}-\frac{n-1}{n} \hat{z}^{i}\right)+\kappa_{0}\left(-n \beta(p)+Z-z^{i}+\hat{z}^{i}\right)^{2} \\
& +p\left(\hat{z}^{i}-\beta(p)\right)+\frac{p^{2}}{4 \kappa_{0} n^{2}} .
\end{aligned}
$$

Following the same steps taken in the proof of Proposition 1, we can show that $\mathcal{E}_{p}=z-\bar{Z}$ when evaluated at the equilibrium $p$ and $\hat{z}^{i}=z^{i}$, for the $\beta(p)=-\hat{a}-\hat{b} p$, consistent with equilibrium. Also, the equilibrium transfers must be

$$
\left(v_{1}+\left(v_{5}+2 v_{3}\right) \bar{Z}\right)\left(z^{i}-\bar{Z}\right),
$$

so it is straightforward to show the formulas for $\beta_{0}, \beta_{1}$ from the previous section apply here as well, for possibly different coefficients $(a, b, c)$.

Returning now to the discrete-time first-order condition, the argument to be maximized when trader $i$ submits an order $x$ and report $\hat{z}^{i}$ is in this case

$$
(1-q)\left(-x \frac{1}{-b(n-1)}((n-1) a+c(Z-z)+x)+V^{+}(z+x, Z)\right)+q \mathcal{E}\left(p, Z, z^{i}, \hat{z}^{i}\right) .
$$

Taking a derivative with respect to $x$, setting this derivative equal to 0 , and using the result 
that $\mathcal{E}_{p}=z-\bar{Z}$ at the equilibrium $p, \hat{z}$, we have

$$
(1-q) \tau_{g}-\frac{q}{b(n-1)}(z-\bar{Z})=0
$$

where

$$
\tau_{g}=-p+\frac{x}{b(n-1)}+\left(\beta_{0}+\beta_{1} \bar{Z}\right)-\frac{\frac{2 \gamma}{r}\left(1-e^{-r \Delta}\right)}{1-e^{-r \Delta}(1-q)(1+c)^{2}}(z+x-\bar{Z}) .
$$

Plugging in $x=a+b p+c z, \bar{Z}=(-a-b p) / c$, and $x=a+b p+c z$, the second-order condition is met if and only if $b<0$. This also implies that $x=c(z-\bar{Z})$, so

$$
(z+x-\bar{Z})=(1+c) z+(1+c) \frac{a+b p}{c} .
$$

The above can thus be rewritten

$$
(1-q) \tau_{h}-\frac{q}{b(n-1)}\left(z+\frac{a+b p}{c}\right)=0,
$$

where

$$
\tau_{h}=-p+\frac{a+b p+c z}{b(n-1)}+\beta_{0}+\beta_{1} \frac{-a-b p}{c}-\frac{\frac{2 \gamma}{r}\left(1-e^{-r \Delta}\right)}{1-e^{-r \Delta}(1-q)(1+c)^{2}}\left((1+c) z+(1+c) \frac{a+b p}{c}\right) .
$$

Gathering terms in $p, z, 1$, we have

$$
\begin{aligned}
& 0=(1-q)\left(-1+\frac{1}{n-1}-\frac{b \beta_{1}}{c}-\frac{\frac{2 \gamma}{r}\left(1-e^{-r \Delta}\right)}{1-e^{-r \Delta}(1-q)(1+c)^{2}}(1+c) \frac{b}{c}\right)-\frac{q}{c(n-1)} \\
& 0=(1-q)\left(\frac{c}{b(n-1)}-\frac{\frac{2 \gamma}{r}\left(1-e^{-r \Delta}\right)}{1-e^{-r \Delta}(1-q)(1+c)^{2}}(1+c)\right)-\frac{q}{b(n-1)} \\
& 0=(1-q)\left(\frac{a}{b(n-1)}+\left(\beta_{0}-\frac{a}{c} \beta_{1}\right)-\frac{\frac{2 \gamma}{r}\left(1-e^{-r \Delta}\right)}{1-e^{-r \Delta}(1-q)(1+c)^{2}}(1+c) \frac{a}{c}\right)-\frac{q a}{b c(n-1)} .
\end{aligned}
$$

We seek $a, b, c, \beta_{1}, \beta_{0}$ such that these three equations and the two equations defining $\beta_{0}, \beta_{1}$ all hold. Conjecture that for some $\tilde{\omega} \in(0,1)$, there is an equilibrium with

$$
a=\frac{r v}{2 \gamma}(1-\tilde{\omega}), \quad b=-\frac{r}{2 \gamma}(1-\tilde{\omega}), \quad c=-(1-\tilde{\omega}) .
$$

Starting with the coefficients on $z$, this means we need

$$
0=(1-q)\left(\frac{2 \gamma}{r(n-1)}-\frac{\frac{2 \gamma}{r}\left(1-e^{-r \Delta}\right)}{1-e^{-r \Delta}(1-q) \tilde{\omega}^{2}} \tilde{\omega}\right)+\frac{2 \gamma q}{r(n-1)(1-\tilde{\omega})}
$$


Multiplying through by $r /(2 \gamma)$, we have

$$
0=(1-q)\left(\frac{1}{(n-1)}-\frac{\left(1-e^{-r \Delta}\right) \tilde{\omega}}{1-e^{-r \Delta}(1-q) \tilde{\omega}^{2}}\right)+\frac{q}{(n-1)(1-\tilde{\omega})} .
$$

Suppose there exists some $\tilde{\omega} \in(0,1)$ satisfying this equality. Straightforward calculation then shows that plugging in $\beta_{0}=v, \beta_{1}=-2 \gamma / r$, the coefficients on $p, 1$ above are all 0 .

Following the steps in the last section, in any equilibrium, we then have

$$
\beta_{1}=\frac{e^{-r \Delta}\left((1-q) \frac{c^{2}}{b}-2 \frac{\gamma}{r}\left(1-e^{-r \Delta}\right) e^{r \Delta}\right)}{1-e^{-r \Delta}(1-q)(1+c)-q e^{-r \Delta}} .
$$

Plugging in the conjectured $a, b, c$, we have

$$
\beta_{1}=\frac{e^{-r \Delta}\left(-\frac{2 \gamma}{r}(1-q)(1-\tilde{\omega})-2 \frac{\gamma}{r}\left(1-e^{-r \Delta}\right) e^{r \Delta}\right)}{1-e^{-r \Delta}(1-q) \tilde{\omega}-q e^{-r \Delta}} .
$$

For $\beta_{1}=-2 \gamma / r$ to be consistent, it must be that

$$
1-e^{-r \Delta}(1-q) \tilde{\omega}-q e^{-r \Delta}=e^{-r \Delta}\left((1-q)(1-\tilde{\omega})+\left(1-e^{-r \Delta}\right) e^{r \Delta}\right) .
$$

But this conditions holds for any $\tilde{\omega}$. Likewise, conjecturing that $\beta_{0}=v$, at the conjectured $a, b, c$, we have

$$
\begin{aligned}
q \beta_{0}+(1-q)\left[\frac{c a}{b}+v\left(1-e^{-r \Delta}\right)(1+c)\right] & =q v+(1-q)\left[v(1-\tilde{\omega})+v\left(1-e^{-r \Delta}\right) \tilde{\omega}\right] \\
& =v\left(1-(1-q) \tilde{\omega} e^{-r \Delta}\right) .
\end{aligned}
$$

Thus $\beta_{0}=v$ is consistent with

$$
\beta_{0}=v\left(1-e^{-r \Delta}\right)+\frac{e^{-r \Delta}\left(q \beta_{0}+(1-q)\left[\frac{c a}{b}+v\left(1-e^{-r \Delta}\right)(1+c)\right]\right)}{1-e^{-r \Delta}(1-q)(1+c)} .
$$

We have thus shown that, as long as $\tilde{\omega}$ satisfies (97), the conjectured $a, b, c$ satisfy the firstorder condition and comprise a subgame perfect equilibrium. In unreported numerical exercises, we find that for sufficiently small $\Delta$ there exists a root $\tilde{\omega}$ such that $-(1-\tilde{\omega}) / \Delta$ is equal to the order-flow coefficient $c$ from Proposition 1, up to machine precision.

\section{G The impaired mechanism}

In this section, we consider an alternate mechanism designed to reduce a fraction $\xi$ of the excess inventory at each implementation. For simplicity, we consider only the case of observable 
aggregate inventory $Z_{t}$. The size-discovery allocations and cash transfers are defined by

$$
Y^{i}(\mu)=\xi\left(\frac{\sum_{j=1}^{n} \mu^{j}}{n}-\mu^{i}\right)
$$

and

$$
\begin{aligned}
T^{i}(\mu, Z) & =\kappa_{0}\left(n \kappa_{2}(Z)+\xi \sum_{j} \mu^{j}\right)^{2}+\kappa_{1}(Z)\left(\xi \mu^{i}+\kappa_{2}(Z)\right)+\frac{\left(2 \xi-\xi^{2}\right) \kappa_{1}^{2}(Z)}{4 n^{2} \kappa_{0}} \\
& +n \kappa_{0} \frac{1-\xi}{\xi}\left[\left(\xi \mu^{i}+\kappa_{2}(Z)\right)^{2}-\left((n-1) \kappa_{2}(Z)+\xi \sum_{j \neq i} \mu^{j}+\frac{\xi \kappa_{1}(Z)}{2 \kappa_{0} n}\right)^{2}\right],
\end{aligned}
$$

for a constant $\kappa_{0}<0$ and affine $\kappa_{1}(\cdot)$ and $\kappa_{2}(\cdot)$. It is worth noting that the sum of these transfers may not be weakly negative for any reports $\mu$. However, we show that, in all the equilibria that we consider, the transfers sum to zero with probability one.

\section{G.1 Sketch of proof of equilibrium}

We provide a sketch of a proof for an alternative version of Proposition 6: For any $\xi \in(0,1]$, there exists a unique symmetric equilibrium such that, each time the mechanism is run, all traders reduce a fraction $\xi$ of their inventory imbalance $z^{i}-\bar{Z}$. The market-clearing price and value functions are identical to those of Proposition 6 , and the exchange demands are identical after replacing $\lambda$ with $\lambda\left(2 \xi-\xi^{2}\right)$. The mechanism reports are still truth-telling: $\mu_{k}^{i}=z_{\tau_{k}}^{i}$. Proof sketch: In any such equilibrium, each trader reports $z^{i}$, so that

$$
Y^{i}(\mu)=\xi\left(\bar{Z}-z^{i}\right)
$$

and the transfers are

$$
\begin{aligned}
T^{i}(\mu, Z)= & \kappa_{0}\left(n \kappa_{2}(Z)+\xi Z\right)^{2}+\kappa_{1}(Z)\left(\xi z^{i}+\kappa_{2}(Z)\right)+\frac{\left(2 \xi-\xi^{2}\right) \kappa_{1}^{2}(Z)}{4 n^{2} \kappa_{0}} \\
& \quad+n \kappa_{0} \frac{1-\xi}{\xi}\left[\left(\xi z^{i}+\kappa_{2}(Z)\right)^{2}-\left((n-1) \kappa_{2}(Z)+\xi\left(Z-z^{i}\right)+\frac{\xi \kappa_{1}(Z)}{2 \kappa_{0} n}\right)^{2}\right] \\
= & \kappa_{0}\left(n \kappa_{2}(Z)+\xi Z\right)^{2}+\kappa_{1}(Z)\left(\xi z^{i}+\kappa_{2}(Z)\right)+\frac{\left(2 \xi-\xi^{2}\right) \kappa_{1}^{2}(Z)}{4 n^{2} \kappa_{0}}+\tau_{a} \tau_{b},
\end{aligned}
$$

where

$$
\begin{aligned}
\tau_{a} & =n \kappa_{0} \frac{1-\xi}{\xi}\left(\xi Z+n \kappa_{2}(Z)+\frac{\xi \kappa_{1}(Z)}{2 \kappa_{0} n}\right) \\
\tau_{b} & =\left(\xi z^{i}+\kappa_{2}(Z)-\left((n-1) \kappa_{2}(Z)+\xi\left(Z-z^{i}\right)+\frac{\xi \kappa_{1}(Z)}{2 \kappa_{0} n}\right)\right) .
\end{aligned}
$$


For any affine $\kappa_{1}, \kappa_{2}$, the transfer can be expressed as

$$
R_{0}+R_{1} Z_{t}+R_{2} Z_{t}^{2}+R_{3} Z_{t} z_{t}^{i}+R_{4} z_{t}^{i}
$$

for constants $R_{0}, \ldots, R_{4}$. Receiving such transfers at independent Poisson arrival times must lead to a linear-quadratic value function, as in the proofs the previous propositions. That is, the equilibrium continuation value function $V$ for trader $i$ must be of the form

$$
V\left(z^{i}, Z\right)=\alpha_{0}^{i}+\alpha_{1} z^{i}+\alpha_{2} \bar{Z}+\alpha_{3}\left(z^{i}\right)^{2}+\alpha_{4} \bar{Z}^{2}+\alpha_{5} z^{i} \bar{Z}
$$

Fixing the assumed equilibrium reports $z^{j}$ for other traders, trader $i$ chooses $\mu$ to maximize

$$
\left(\alpha_{1}+\alpha_{5} \bar{Z}\right) Y^{i}\left(\left(\mu, z^{-i}\right)\right)+\alpha_{3} Y^{i}\left(\left(\mu, z^{-i}\right)\right)^{2}+2 \alpha_{3} Y^{i}\left(\left(\mu, z^{-i}\right)\right) z^{i}+T^{i}\left(\left(\mu, z^{-i}\right), Z\right),
$$

where, writing $\kappa_{2}(Z)=\hat{a}+\hat{b} Z$,

$$
\begin{aligned}
T^{i}\left(\left(\mu, z^{-i}\right), Z\right) & =\kappa_{0}\left(\xi \mu+n \hat{a}+n \hat{b} Z+\xi\left(Z-z^{i}\right)\right)^{2}+\kappa_{1}(Z)(\xi \mu+\hat{a}+\hat{b} Z)+\frac{\left(2 \xi-\xi^{2}\right) \kappa_{1}^{2}(Z)}{4 n^{2} \kappa_{0}} \\
& +n \kappa_{0} \frac{1-\xi}{\xi}\left[\left(\xi z^{i}+\hat{a}+\hat{b} Z\right)^{2}-\left((n-1)(\hat{a}+\hat{b} Z)+\xi \sum_{j \neq i} z^{j}+\frac{\xi \kappa_{1}(Z)}{2 \kappa_{0} n}\right)^{2}\right] .
\end{aligned}
$$

The first-order condition is

$$
\begin{aligned}
& -\frac{n-1}{n} \xi\left(\alpha_{1}+\alpha_{5} \bar{Z}+2 \alpha_{3} z^{i}\right)-\frac{2(n-1) \alpha_{3} \xi}{n} Y^{i}\left(\left(\mu, z^{-i}\right)\right)+\xi \kappa_{1}(Z) \\
& \quad+2 \kappa_{0} \xi\left(\xi \mu+n \hat{a}+n \hat{b} Z+\xi\left(Z-z^{i}\right)\right)+2 n \kappa_{0} \xi \frac{1-\xi}{\xi}\left(\xi z^{i}+\hat{a}+\hat{b} Z\right)=0 .
\end{aligned}
$$

Plugging in $\mu=z^{i}$ and $Y^{i}\left(\left(\mu, z^{-i}\right)\right)=\xi\left(\bar{Z}-z^{i}\right)$, and then dividing through by $\xi$, we have

$$
\begin{gathered}
-\frac{n-1}{n}\left(\alpha_{1}+\alpha_{5} \bar{Z}+2 \alpha_{3} z^{i}\right)-\frac{2(n-1) \alpha_{3}}{n} \xi\left(\bar{Z}-z^{i}\right)+\kappa_{1}(Z) \\
+2 \kappa_{0}(n \hat{a}+n \hat{b} Z+\xi Z)+2 n \kappa_{0} \frac{1-\xi}{\xi}\left(\xi z^{i}+\hat{a}+\hat{b} Z\right)=0 .
\end{gathered}
$$

It is clear that the terms involving $z^{i}$ cancel if and only if $\kappa_{0}=(n-1) \alpha_{3} / n^{2}$. Given this, 
the unique $\hat{a}, \hat{b}$ solving this must satisfy

$$
\begin{aligned}
0= & -\frac{n-1}{n}\left(\alpha_{1}+\alpha_{5} \bar{Z}\right)-\frac{2(n-1) \alpha_{3}}{n} \xi(\bar{Z})+\kappa_{1}(Z) \\
& \left.+\frac{2(n-1) \alpha_{3}}{n^{2}}(n \hat{a}+n \hat{b} Z+\xi Z)\right)+\frac{2(n-1) \alpha_{3}}{n} \frac{1-\xi}{\xi}(\hat{a}+\hat{b} Z), \\
\hat{a}+\hat{b} Z= & \frac{n \xi}{2(n-1) \alpha_{2}}\left(-\kappa_{1}(Z)+\left(\alpha_{1}+\alpha_{5} \bar{Z}\right) \frac{n-1}{n}\right) \\
= & \frac{\xi}{2 n \kappa_{0}}\left(-\kappa_{1}(Z)+\left(\alpha_{1}+\alpha_{5} \bar{Z}\right) \frac{n-1}{n}\right) .
\end{aligned}
$$

Manipulating the formula for transfers, we can write the equilibrium transfer to trader $i$, given $\mu^{i}=z^{i}$ for all $i$, as

$$
\kappa_{0}\left(n \kappa_{2}(Z)+\xi Z\right)^{2}+\kappa_{1}(Z)\left(\xi z^{i}+\kappa_{2}(Z)\right)+\frac{\left(2 \xi-\xi^{2}\right) \kappa_{1}^{2}(Z)}{4 n^{2} \kappa_{0}}+\tau_{a} \tau_{b} .
$$

Defining $\kappa_{1}(\cdot)$ so that

$$
\xi Z+n \kappa_{2}(Z)+\frac{\xi \kappa_{1}(Z)}{2 \kappa_{0} n}=0
$$

this transfer simplifies to

$$
\kappa_{0}\left(n \kappa_{2}(Z)+\xi Z\right)^{2}+\kappa_{1}(Z)\left(\xi z^{i}+\kappa_{2}(Z)\right)+\frac{\left(2 \xi-\xi^{2}\right) \kappa_{1}^{2}(Z)}{4 n^{2} \kappa_{0}} .
$$

The sum of the transfers across all traders is

$$
n \kappa_{0}\left(\frac{\xi \kappa_{1}(Z)}{2 \kappa_{0} n}\right)^{2}-\kappa_{1}(Z)\left(\frac{\xi \kappa_{1}(Z)}{2 \kappa_{0} n}\right)+\frac{\left(2 \xi-\xi^{2}\right) \kappa_{1}^{2}(Z)}{4 n \kappa_{0}}=0 .
$$

Some calculation shows that the above choice for $\kappa_{1}(\cdot)$ uniquely ensures that the transfers sum to zero with probability 1 , which must be the case for IR and budget balance to hold. Plugging in the formula for $\kappa_{2}(\cdot)$, we see that we need the conditions

$$
\begin{aligned}
0 & =\xi Z+\frac{\xi}{2 \kappa_{0}}\left(-\kappa_{1}(Z)+\left(\alpha_{1}+\alpha_{5} \bar{Z}\right) \frac{n-1}{n}\right)+\frac{\xi \kappa_{1}(Z)}{2 \kappa_{0} n} \\
0 & =2 \kappa_{0} n Z+\left(-n \kappa_{1}(Z)+\left(\alpha_{1}+\alpha_{5} \bar{Z}\right)(n-1)\right)+\kappa_{1}(Z) \\
\kappa_{1}(Z) & =\left(\alpha_{1}+\alpha_{5} \bar{Z}\right)+\frac{2 \kappa_{0} n}{n-1} Z \\
& =\alpha_{1}+\left(\alpha_{5}+2 \alpha_{3}\right) \bar{Z} .
\end{aligned}
$$

This is the unique choice for $\kappa_{1}(Z)$ consistent with budget balance and ex-post IR. 
The HJB equation is

$$
\begin{aligned}
r V\left(z^{i}, Z\right)=- & \gamma\left(z^{i}\right)^{2}+r v z+\frac{\sigma_{i}^{2}}{2} V_{z z}\left(z^{i}, Z\right)+\frac{\sigma_{Z}^{2}}{2} V_{Z Z}\left(z^{i}, Z\right)+\rho^{i} V_{z Z}\left(z^{i}, Z\right) \\
& +\sup _{D, \mu}\left\{-\Phi_{(a, b, c)}\left(D ; Z-z^{i}\right) D+V_{z}\left(z^{i}, Z\right) D\right. \\
& \left.+\lambda\left(V\left(z^{i}+Y^{i}\left(\mu, z^{-i}\right), Z\right)-V\left(z^{i}, Z\right)+T^{i}\left(\left(\mu, z^{-i}\right), Z\right)\right)\right\} .
\end{aligned}
$$

We just showed that because $V$ is linear-quadratic, at the unique candidate equilibrium reallocations we must have

$$
V\left(z+Y^{i}\left(\mu, z^{-i}\right), Z\right)-V(z, Z)=\left(\alpha_{1}+\alpha_{5} \bar{Z}\right) \xi(\bar{Z}-z)+\alpha_{3} \xi^{2}(\bar{Z}-z)^{2}+2 \alpha_{3} \xi z(\bar{Z}-z) .
$$

By the above, the equilibrium transfer is

$$
\kappa_{0}\left(\frac{\xi \kappa_{1}(Z)}{2 \kappa_{0} n}\right)^{2}+\kappa_{1}(Z)\left(\xi\left(z^{i}-\bar{Z}\right)-\frac{\xi \kappa_{1}(Z)}{2 \kappa_{0} n}\right)+\frac{\left(2 \xi-\xi^{2}\right) \kappa_{1}^{2}(Z)}{4 n^{2} \kappa_{0}}=\kappa_{1}(Z) \xi\left(z^{i}-\bar{Z}\right) .
$$

Plugging in $\kappa_{1}(Z)=\alpha_{1}+\left(\alpha_{5}+2 \alpha_{3}\right) \bar{Z}$ and summing the transfer and the change in continuation value gives

$$
\begin{aligned}
\left(\alpha_{1}+\alpha_{5} \bar{Z}\right) \xi(\bar{Z}-z) & +\alpha_{3} \xi^{2}(\bar{Z}-z)^{2}+2 \alpha_{3} \xi z(\bar{Z}-z)-\left(\alpha_{1}+\alpha_{5} \bar{Z}+2 \alpha_{3} \bar{Z}\right) \xi(\bar{Z}-z) \\
& =\alpha_{3} \xi^{2}(\bar{Z}-z)^{2}-2 \alpha_{3} \xi\left(z^{2}+\bar{Z}^{2}-2 z \bar{Z}\right) \\
& =-\alpha_{3}\left(2 \xi-\xi^{2}\right)(\bar{Z}-z)^{2}
\end{aligned}
$$

Plugging this in, the reduced HJB equation is

$$
\begin{aligned}
r V\left(z^{i}, Z\right) & =-\gamma\left(z^{i}\right)^{2}+r v z^{i}+\frac{\sigma_{i}^{2}}{2} V_{z z}\left(z^{i}, Z\right)+\frac{\sigma_{Z}^{2}}{2} V_{Z Z}\left(z^{i}, Z\right)+\rho^{i} V_{z Z}\left(z^{i}, Z\right) \\
& +\sup _{D}\left\{-\Phi_{(a, b, c)}\left(D ; Z-z^{i}\right) D+V_{z}\left(z^{i}, Z\right) D-\lambda\left(2 \xi-\xi^{2}\right) \alpha_{3}\left(z^{i}-\bar{Z}\right)^{2}\right\} .
\end{aligned}
$$

This is exactly the HJB equation found in the proof of Proposition 6, after replacing $\lambda$ with $\lambda^{*}=\lambda\left(2 \xi-\xi^{2}\right)$.

\section{H Only size discovery: observable $Z_{t}$}

In the main text of the paper, we showed that augmenting a price-discovery market with future size-discovery sessions never increases welfare, and strictly reduces welfare if the size-discovery platform operator relies on the price-discovery market for information about aggregate inventory imbalances. It is then natural to ask whether simply getting rid of the price-discovery market, and running only size-discovery sessions, could improve welfare, relative to a setting with price discovery. When stand-alone size discovery is feasible and is run sufficiently frequently, and the aggregate excess inventory $Z_{t}$ is observable, it strictly improves welfare, and indeed is strictly preferred by each trader individually. From a practical viewpoint, however, it could be difficult to arrange for the abandonment of price-discovery markets. Moreover, 
the size-discovery sessions that we analyze might be difficult to implement in practice without information coming out of the price-discovery market.

In this appendix, we consider a pure size-discovery market, for an economy with observable aggregate inventory. We exploit the same perfect-reallocation size-discovery sessions developed earlier. As before, these sessions are run at the event times of an independent Poisson process $N$ with mean arrival rate $\lambda>0$.

Again, traders submit mechanism report processes $\mu=\left(\mu^{1}, \ldots, \mu^{n}\right)$. The resulting excessinventory process $z^{i}$ of trader $i$ is then determined by

$$
z_{t}^{i}=z_{0}^{i}+H_{t}^{i}+\sum_{\left\{k: \tau_{k}<\min (\mathcal{T}, t)\right\}} \frac{\sum_{j=1}^{n} \mu_{k}^{j}}{n}-\mu_{k}^{i}
$$

There is no exchange market price to be observed, but the aggregate inventory $Z_{t}$ is assumed to be common knowledge for all $t$. The size-discovery mechanism design $\left(Y, T_{\kappa}\right)$ uses the asset reallocation determined by (25). We again apply the cash-transfer function $T_{\kappa}$ defined by (26) for some coefficient $\kappa_{0}<0$, with

$$
\kappa_{1}\left(Z_{t}\right)=v-\frac{2 \gamma}{r} \bar{Z}_{t}
$$

and

$$
\kappa_{2}\left(Z_{t}\right)=-\bar{Z}_{t}-\frac{\kappa_{1}\left(Z_{t}\right)}{2 \kappa_{0} n^{2}}
$$

By the same reasoning provided in Appendix A, one can show these are the unique affine choices for $\kappa_{1}(\cdot)$ and $\kappa_{2}(\cdot)$ such that an equilibrium exists. Moreover, we must restrict attention to affine $\kappa_{1}(\cdot), \kappa_{2}(\cdot)$ in this dynamic setting in order to guarantee a linear-quadratic continuation-value function.

We seek a truth-telling equilibrium of the dynamic reporting game, in which each trader optimally chooses to report $\mu_{k}^{j}=z^{j}\left(\tau_{k}\right)$ and in which mechanism participation is always individually rational. The exact stochastic control problem solved by each trader is an obvious simplification of the control problem of Appendix D. The next proposition confirms that this equilibrium exists and provides a calculation of the continuation value for each trader.

Proposition 7. For any $\kappa_{0}<0$, consider the size-discovery session mechanism design $\left(Y, T_{\kappa}\right)$ of (25)-(26), with (101)-(102). The truth-telling equilibrium, that with reports $\mu_{k}^{i}=z_{\tau_{k}}^{i}$, exists and has the following properties.

1. At each session time $\tau_{k}$, each trader $i$ achieves the efficient post-session position $z^{i}\left(\tau_{k}\right)=$ $\bar{Z}\left(\tau_{k}\right)$, almost surely.

2. For each trader $i$, the equilibrium continuation value $V_{M}^{i}\left(z_{t}^{i}, Z_{t}\right)$ at time $t$ is

$$
V_{M}^{i}\left(z_{t}^{i}, Z_{t}\right)=\tilde{\theta}_{i}+v \bar{Z}_{t}-\frac{\gamma}{r} \bar{Z}_{t}^{2}+\kappa_{1}\left(Z_{t}\right)\left(z_{t}^{i}-\bar{Z}_{t}\right)-\frac{\gamma}{r+\lambda}\left(z_{t}^{i}-\bar{Z}_{t}\right)^{2}
$$

where

$$
\tilde{\theta}_{i}=\frac{1}{r}\left(\frac{\gamma}{r} \frac{\sigma_{Z}^{2}}{n^{2}}-\frac{\gamma}{r+\lambda}\left(\frac{\sigma_{Z}^{2}}{n^{2}}+\sigma_{i}^{2}-2 \frac{\rho^{i}}{n}\right)-\frac{2 \gamma}{r} \frac{\rho^{i}}{n}\right) .
$$


As the mean frequency $\lambda$ of reallocation sessions approaches infinity, the equilibrium welfare approaches the first-best welfare $W_{f b}(Z)$. This follows from the fact that the equilibrium total expected holding costs associated with excess inventory, relative to the holding costs at first best, approaches zero ${ }^{40}$ as $\lambda \rightarrow \infty$. This is immediate from the fact that the quadratic coefficient $\gamma /(r+\lambda)$ of the indirect utility $V_{M}^{i}$ approaches zero as $\lambda \rightarrow \infty$. These properties hold for any choice of $\kappa_{0}<0$, but setting $\kappa_{0}=-\gamma(n-1) /\left(n^{2}(r+\lambda)\right)$ makes each trader indifferent to instantaneous deviations by other traders. ${ }^{41}$

\section{H.1 Proof of Proposition 7}

The proof is extremely similar to that of Proposition 6, so we leave some details to the reader. We write $V(z, Z)$ rather than $V_{M}^{i}(z, Z)$ for brevity. For any affine $\kappa_{1}(\cdot)$ and $\kappa_{2}(\cdot)$, the transfers in equilibrium take the form

$$
R_{0}+R_{1} Z_{t}+R_{2} Z_{t}^{2}+R_{3} Z_{t} z_{t}^{i}+R_{4} z_{t}^{i}
$$

for some constants $R_{0}$ through $R_{4}$. In any symmetric equilibrium, the value function

$$
V\left(z_{0}^{i}, Z_{0}\right)=\mathbb{E}\left[\pi z_{\mathcal{T}}^{i}+\int_{0}^{\mathcal{T}}-\gamma\left(z_{s}^{i}\right)^{2} d s+\sum_{\left\{k: \tau_{k}<\mathcal{T}\right\}} T_{\kappa}^{i}\left(\mu_{k}^{i}, Z_{\tau_{k}}\right)\right]
$$

takes the form

$$
V(z, Z)=\alpha_{0}^{i}+\alpha_{1} z+\alpha_{2} \bar{Z}+\alpha_{3} z^{2}+\alpha_{4} \bar{Z}^{2}+\alpha_{5} z \bar{Z}
$$

where

$$
\begin{aligned}
\alpha_{3} & =\frac{-\gamma}{r+\lambda} \\
\alpha_{5} & =\frac{1}{r+\lambda}\left(\lambda n R_{3}\right) \\
\alpha_{4} & =\frac{1}{r}\left(\lambda \alpha_{5}+\lambda \alpha_{3}+\lambda n^{2} R_{2}\right) \\
\alpha_{1} & =\frac{1}{r+\lambda}\left(r v+\lambda R_{4}\right) \\
\alpha_{2} & =\frac{1}{r}\left(\lambda \alpha_{1}+\lambda n R_{1}\right) \\
\alpha_{0}^{i} & =\frac{1}{r}\left(\alpha_{3} \sigma_{i}^{2}+\alpha_{4} \frac{\sigma_{Z}^{2}}{n^{2}}+\alpha_{5} \frac{\rho^{i}}{n}+\lambda R_{0}\right),
\end{aligned}
$$

\footnotetext{
${ }^{40}$ This convergence is also intuitively obvious from the fact that $\delta_{t}^{i} \equiv\left(z_{t}^{i}-\bar{Z}_{t}\right)^{2}$ jumps to zero at each of the event times of $N$. The duration of time between these successive perfect reallocations has expectation $1 / \lambda$, which goes to zero. Between these perfect reallocations, $\delta_{t}^{i}$ has a mean that is continuous in $t$ and grows in expectation at a bounded rate.

${ }^{41}$ Formally, if we consider the static mechanism report game with the continuation value corresponding to Proposition 7 , for this $\kappa_{0}$ truth-telling is a dominant strategy.
} 
and where $R_{0}$ through $R_{4}$ are the previously defined transfer coefficients. To see this, note that given the $\alpha$ coefficients, we have

$$
\begin{aligned}
(r+\lambda)\left(\alpha_{0}^{i}+\alpha_{1} z+\alpha_{2} \bar{Z}+\alpha_{3} z^{2}+\alpha_{4} \bar{Z}^{2}+\alpha_{5} z \bar{Z}\right)=r v z-\gamma z^{2}+\alpha_{4} \frac{\sigma_{Z}^{2}}{n^{2}}+\alpha_{3} \sigma_{i}^{2}+\alpha_{5} \frac{\rho^{i}}{n} \\
+\lambda\left(\alpha_{0}^{i}+\alpha_{1} \bar{Z}+\alpha_{2} \bar{Z}+\alpha_{3} \bar{Z}^{2}+\alpha_{4} \bar{Z}^{2}+\alpha_{5} \bar{Z}^{2}+R_{0}+R_{1} Z+R_{2} Z^{2}+R_{3} Z z+R_{4} z\right)
\end{aligned}
$$

Let $Y_{t}=1_{\{\mathcal{T} \leq t\}}$ and $V(z, Z)$ be defined as above, and let $U_{t}=\left(1-Y_{t}\right) V\left(z_{t}^{i}, Z_{t}\right)+Y_{t} v z_{t}^{i}$. Following the steps of the proof of Proposition 6, letting

$$
\chi_{s}=\alpha_{4} \frac{\sigma_{Z}^{2}}{n^{2}}+\alpha_{3} \sigma_{i}^{2}+\alpha_{5} \frac{\rho^{i}}{n}-\lambda\left(z_{s}^{i}-\bar{Z}_{s}\right)\left(\alpha_{1}+\alpha_{5} \bar{Z}_{s-}+\alpha_{3}\left(z_{s}^{i}+\bar{Z}_{s}\right)\right)+r\left(v z_{s}^{i}-V\left(z_{s}^{i}, Z_{s}\right)\right),
$$

we can show that

$$
\mathbb{E}\left(U_{\mathcal{T}}-U_{0}\right)=\mathbb{E}\left[\int_{0}^{\mathcal{T}} \chi_{s} d s\right] .
$$

Because $\alpha_{0}^{i}$ through $\alpha_{5}$ satisfy the system of equations specified at the beginning of this proof, we have

$$
\mathbb{E}\left(U_{\mathcal{T}}-U_{0}\right)=\mathbb{E}\left[\int_{0}^{\mathcal{T}} \bar{\chi}_{s} d s\right]
$$

where

$$
\bar{\chi}_{s}=\gamma\left(z_{s}^{i}\right)^{2}-\lambda\left(R_{0}+R_{1} Z_{s}+R_{2} Z_{s}^{2}+R_{3} Z_{s} z_{s}^{i}+R_{4} z_{s}^{i}\right) .
$$

Using the definitions of $U, \mathcal{T}$, and $R_{0}$ through $R_{4}$, as well as the fact that $\mathbb{E}\left(v z_{\mathcal{T}}^{i}\right)=\mathbb{E}\left(\pi z_{\mathcal{T}}^{i}\right)$, we can rearrange to find that

$$
\begin{aligned}
V\left(z_{0}^{i}, Z_{0}\right) & =\mathbb{E}\left[\pi z_{\mathcal{T}}^{i}+\int_{0+}^{\mathcal{T}} \bar{\chi}_{s} d s\right] \\
& =\mathbb{E}\left[\pi z_{\mathcal{T}}^{i}+\int_{0+}^{\mathcal{T}}-\gamma\left(z_{s}^{i}\right)^{2}+\lambda T_{\kappa}^{i}\left(\hat{\mu}_{s}, Z_{s}\right) d s\right] \\
& =\mathbb{E}\left[\pi z_{\mathcal{T}}^{i}+\int_{0}^{\mathcal{T}}-\gamma\left(z_{s}^{i}\right)^{2} d s+\int_{0}^{\mathcal{T}} T_{\kappa}^{i}\left(\hat{\mu}_{s}, Z_{s}\right) d N_{s}\right]
\end{aligned}
$$

where $\hat{\mu}$ is the optional projection of the report process. This shows that the value function $V(z, Z)$ takes the form suggested above. The same arguments used in Appendix D now go through (up to these different $\alpha$ coefficients), so it must be that

$$
\kappa_{1}(Z)=\alpha_{1}+\left(\alpha_{5}+2 \alpha_{3}\right) \bar{Z} \text {. }
$$

and the equilibrium reports are optimal provided that

$$
\kappa_{2}(Z)=\hat{a}+\hat{b} Z=-\bar{Z}-\frac{\alpha_{1}+\left(\alpha_{5}+2 \alpha_{3}\right) \bar{Z}}{2 \kappa_{0} n^{2}} .
$$


Once again the equilibrium transfers are $\left(\alpha_{1}+\left(\alpha_{5}+2 \alpha_{3}\right) \bar{Z}\right)\left(z^{i}-\bar{Z}\right)$, so the coefficients $R_{m}$ in

$$
R_{0}+R_{1} Z_{t}+R_{2} Z_{t}^{2}+R_{3} Z_{t} z_{t}^{i}+R_{4} z_{t}^{i}
$$

are given by

$$
\begin{aligned}
& R_{0}=0 \\
& R_{1}=-\frac{\alpha_{1}}{n} \\
& R_{2}=-\frac{\alpha_{5}+2 \alpha_{3}}{n^{2}} \\
& R_{3}=\frac{\alpha_{5}+2 \alpha_{3}}{n} \\
& R_{4}=\alpha_{1} .
\end{aligned}
$$

From the above, we have that

$$
\begin{aligned}
\alpha_{3} & =\frac{-\gamma}{r+\lambda} \\
\alpha_{5} & =\frac{1}{r+\lambda}\left(\lambda n R_{3}\right) \\
\alpha_{4} & =\frac{1}{r}\left(\lambda \alpha_{5}+\lambda \alpha_{3}+\lambda n^{2} R_{2}\right) \\
\alpha_{1} & =\frac{1}{r+\lambda}\left(r v+\lambda R_{4}\right) \\
\alpha_{2} & =\frac{1}{r}\left(\lambda \alpha_{1}+\lambda n R_{1}\right) .
\end{aligned}
$$

So, plugging in $R_{1}, R_{2}, R_{3}, R_{4}$, and rearranging, we have

$$
\begin{aligned}
\alpha_{3} & =\frac{-\gamma}{r+\lambda} \\
\alpha_{5} & =\frac{1}{r}\left(2 \lambda \alpha_{3}\right)=\frac{2 \lambda}{r}\left(\frac{-\gamma}{r+\lambda}\right) \\
\alpha_{4} & =\frac{1}{r}\left(\lambda \alpha_{5}+\lambda \alpha_{3}-\lambda\left(\alpha_{5}+2 \alpha_{3}\right)\right)=\frac{\lambda}{r}\left(\frac{\gamma}{r+\lambda}\right) \\
\alpha_{1} & =\frac{1}{r}(r v)=v \\
\alpha_{2} & =\frac{1}{r}\left(\lambda \alpha_{1}-\lambda \alpha_{1}\right)=0 .
\end{aligned}
$$

With these choices for $\alpha_{1}$ through $\alpha_{5}$, and with

$$
\alpha_{0}^{i}=\frac{1}{r}\left(\alpha_{3} \sigma_{i}^{2}+\alpha_{4} \frac{\sigma_{Z}^{2}}{n^{2}}+\alpha_{5} \frac{\rho^{i}}{n}\right),
$$


we can define the value function

$$
V\left(z^{i}, Z\right)=\alpha_{0}^{i}+\alpha_{1} z^{i}+\alpha_{2} \bar{Z}+\alpha_{3}\left(z^{i}\right)^{2}+\alpha_{4} \bar{Z}^{2}+\alpha_{5} z^{i} \bar{Z}
$$

This value function solves the associated HJB equation

$$
\begin{aligned}
0=- & \gamma\left(z^{i}\right)^{2}+r\left(v z^{i}-V\left(z^{i}, Z\right)\right)+\frac{\sigma_{i}^{2}}{2} V_{z z}\left(z^{i}, Z\right)+\frac{\sigma_{Z}^{2}}{2} V_{Z Z}\left(z^{i}, Z\right)+\rho^{i} V_{z Z}\left(z^{i}, Z\right) \\
& +\sup _{\mu}\left\{\lambda\left(V\left(z^{i}+Y^{i}\left(\left(\mu, z^{-i}\right)\right), Z\right)-V\left(z^{i}, Z\right)+T_{\kappa}^{i}\left(\left(\mu, z^{-i}\right), Z\right)\right)\right\} .
\end{aligned}
$$

Plugging in $\alpha_{1}, \alpha_{3}, \alpha_{5}$, we have

$$
\kappa_{1}(Z)=v-\frac{2 \gamma}{r} \bar{Z}
$$

and

$$
\kappa_{2}(Z)=-\bar{Z}-\frac{v-\frac{2 \gamma}{r} \bar{Z}}{2 \kappa_{0} n^{2}} .
$$

The last part of the verification, demonstrating that alternative strategies do weakly worse, is exactly the same as in the verification proof of Appendix D, and thus omitted. Rearranging the coefficients $\alpha_{0}^{i}$ through $\alpha_{5}$ above gives the proposed expression for $V$, completing the proof.

\section{Only size discovery: unobservable $Z_{t}$}

This appendix demonstrates that a version of our mechanism can achieve the first-best allocation in our dynamic setting, even when $Z_{t}$ is unobserved, if the mechanism is run continuously and there is no exchange market. However, as we will show, it is not individually rational for participants to enter this mechanism. We only provide a sketch of this proof, since the technical details are similar to the proofs in the previous appendices.

A size-discovery reporting process in this setting is a finite-variance progressively measurable process $\hat{z}$. If the traders' respective reporting processes are $\hat{z}=\left(\hat{z}^{1}, \ldots, \hat{z}^{n}\right)$, then the excess inventory of trader $i$ is

$$
z_{t}^{i}=z_{0}^{i}+Y^{i}\left(\hat{z}_{t}\right)+H_{t}^{i}
$$

where

$$
Y^{i}\left(\hat{z}_{t}\right)=\frac{\sum_{j=1}^{n} \hat{z}_{t}^{j}}{n}-\hat{z}_{t}^{i} .
$$

We assume that trader $i$ is continuously compensated, where the flow payment is determined by some measurable transfer function $\tilde{T}_{\kappa}^{i}: \mathbb{R}^{n} \rightarrow \mathbb{R}$ that is bounded by a second-order polynomial. Thus, each trader $i$ takes the reporting strategies $\hat{z}_{t}^{-i}$ of the other traders as given, and chooses a report process $\tilde{z}$ to solve

$$
V^{i}\left(z_{0}^{i}, Z\right)=\sup _{\tilde{z}} \mathbb{E}\left[z_{\mathcal{T}}^{\tilde{z}} \pi+\int_{0}^{\mathcal{T}} \tilde{T}_{\kappa}^{i}\left(\left(\tilde{z}_{t}, \hat{z}_{t}^{-i}\right)\right)-\gamma\left(z_{t}^{\tilde{z}}\right)^{2} d t\right]
$$


subject to

$$
z_{t}^{\tilde{z}}=z_{0}^{i}+Y^{i}\left(\left(\tilde{z}_{t}, \hat{z}_{t}^{-i}\right)\right)+H_{t}^{i}
$$

We now simplify the problem. By conditioning on everything except $\pi$ and applying the tower property, by independence we may rewrite the objective as

$$
\sup _{\tilde{z}} \mathbb{E}\left[z_{\mathcal{T}}^{\tilde{z}} v+\int_{0}^{\mathcal{T}} \tilde{T}_{\kappa}^{i}\left(\left(\tilde{z}_{t}, \hat{z}_{t}^{-i}\right)\right)-\gamma\left(z_{t}^{\tilde{z}}\right)^{2} d t\right] .
$$

By conditioning on everything except $\mathcal{T}$ and applying the tower property, by independence we may rewrite this as

$$
\sup _{\tilde{z}} \mathbb{E}\left[\int_{0}^{\infty} r v e^{-r u} z_{u}^{\tilde{z}} d u+\int_{0}^{\infty} r e^{-r u} \int_{0}^{u} \tilde{T}_{\kappa}^{i}\left(\left(\tilde{z}_{t}, \hat{z}_{t}^{-i}\right)\right)-\gamma\left(z_{t}^{\tilde{z}}\right)^{2} d t d u\right] .
$$

Applying a change of order of integration, this is

$$
\begin{aligned}
& \sup _{\tilde{z}} \mathbb{E}\left[\int_{0}^{\infty} r v e^{-r u} z_{u}^{\tilde{z}} d u+\int_{0}^{\infty}\left(\tilde{T}_{\kappa}^{i}\left(\left(\tilde{z}_{t}, \hat{z}_{t}^{-i}\right)\right)-\gamma\left(z_{t}^{\tilde{z}}\right)^{2}\right) \int_{t}^{\infty} r e^{-r u} d u d t\right] \\
& =\sup _{\tilde{z}} \mathbb{E}\left[\int_{0}^{\infty} r v e^{-r u} z_{u}^{\tilde{z}} d u+\int_{0}^{\infty}\left(\tilde{T}_{\kappa}^{i}\left(\left(\tilde{z}_{t}, \hat{z}_{t}^{-i}\right)\right)-\gamma\left(z_{t}^{\tilde{z}}\right)^{2}\right)\left(e^{-r t}\right) d t\right] \\
& =\sup _{\tilde{z}} \mathbb{E}\left[\int_{0}^{\infty} e^{-r t}\left(r v z_{t}^{\tilde{z}}+\tilde{T}_{\kappa}^{i}\left(\left(\tilde{z}_{t}, \hat{z}_{t}^{-i}\right)\right)-\gamma\left(z_{t}^{\tilde{z}}\right)^{2}\right) d t\right] .
\end{aligned}
$$

Define $\nu_{t} \equiv z_{0}^{i}+H_{t}^{i}$, which does not depend on $\tilde{z}_{t}$. Then plugging in (106) to this new objective gives

$$
\sup _{\tilde{z}} \mathbb{E}\left[\int_{0}^{\infty} e^{-r t}\left(r v\left[\nu_{t}+Y^{i}\left(\left(\tilde{z}_{t}, \hat{z}_{t}^{-i}\right)\right)\right]+\tilde{T}_{\kappa}^{i}\left(\left(\tilde{z}_{t}, \hat{z}_{t}^{-i}\right)\right)-\gamma\left(\left[\nu_{t}+Y^{i}\left(\left(\tilde{z}_{t}, \hat{z}_{t}^{-i}\right)\right)\right]\right)^{2}\right) d t\right] .
$$

By additivity, if $\tilde{z}(\omega, t)$ solves, at each $(\omega, t)$, the problem

$$
\sup _{\tilde{z}_{t}} e^{-r t}\left(r v\left[\nu_{t}+Y^{i}\left(\left(\tilde{z}_{t}, \hat{z}_{t}^{-i}\right)\right)\right]+\tilde{T}_{\kappa}^{i}\left(\left(\tilde{z}_{t}, \hat{z}_{t}^{-i}\right)\right)-\gamma\left(\left[\nu_{t}+Y^{i}\left(\left(\tilde{z}_{t}, \hat{z}_{t}^{-i}\right)\right)\right]\right)^{2}\right)
$$

then the process $\tilde{z}$ solves the dynamic optimization problem. Now, we let

$$
\begin{aligned}
V_{\text {static }}^{i}\left(z^{i}, Z\right) & =u_{\text {static }}^{i}(Z)+\left(\beta_{0}+\beta_{1} \bar{Z}\right)\left(z^{i}-\bar{Z}\right)-K\left(z^{i}-\bar{Z}\right)^{2} \\
\beta_{0} & =r v \\
\beta_{1} & =-2 \gamma \\
K & =\gamma \\
u_{\text {static }}^{i}(Z) & =r v \bar{Z}-\gamma \bar{Z}^{2} .
\end{aligned}
$$

Because multiplying by $e^{r t}$ does not change the optimization, problem (107) is strategically equivalent to

$$
\sup _{\tilde{z}} V_{\text {static }}^{i}\left(\nu_{t}+Y^{i}\left(\left(\tilde{z}, \hat{z}_{t}^{-i}\right)\right), Z_{t}\right)+\tilde{T}_{\kappa}^{i}\left(\left(\tilde{z}, \hat{z}_{t}^{-i}\right)\right) .
$$


Let

$$
\begin{aligned}
\kappa_{0} & \equiv-K(n-1) / n^{2}=-\gamma(n-1) / n^{2} \\
\kappa_{1}(Z) & \equiv \kappa_{1}
\end{aligned}
$$

for any constant $\kappa_{1}$. Then it is immediate from Appendix A that if we define

$$
\begin{aligned}
\kappa_{2}(Z) & =-\bar{Z}+\frac{-\kappa_{1}(Z)+\left(\frac{n-1}{n}\right)\left(\beta_{0}+\beta_{1} \bar{Z}\right)}{2 \kappa_{0} n} \\
& =\frac{-\kappa_{1}+\left(\frac{n-1}{n}\right) r v}{2 \kappa_{0} n} \\
& =-\frac{-n \kappa_{1}+(n-1) r v}{2 \gamma(n-1)}=\kappa_{2}
\end{aligned}
$$

and

$$
\tilde{T}_{\kappa}^{i}(\hat{z})=\kappa_{1} \hat{z}^{i}+\kappa_{0}\left(n \kappa_{2}+\sum_{j=1}^{n} \hat{z}^{j}\right)^{2}+\kappa_{1} \kappa_{2}+\frac{\kappa_{1}^{2}}{4 \kappa_{0} n^{2}},
$$

then it is a strictly dominant strategy for each trader to report $\tilde{z}_{t}=\nu_{t}=z_{0}^{i}+H_{t}^{i}$. Further, just as in the main text of the paper, the sum of the transfers in each instant is weakly negative:

$$
\begin{aligned}
\sum_{i=1}^{n} \tilde{T}_{\kappa}^{i}(\hat{z}) & =\kappa_{1} \sum_{j=1}^{n} \hat{z}^{j}+n \kappa_{0}\left(n \kappa_{2}+\sum_{j=1}^{n} \hat{z}^{j}\right)^{2}+n \kappa_{1} \kappa_{2}+\frac{\kappa_{1}^{2}}{4 \kappa_{0} n} \\
& =\frac{1}{4 \kappa_{0} n}\left(\kappa_{1}+2 \kappa_{0} n\left(n \kappa_{2}+\sum_{j=1}^{n} \hat{z}^{j}\right)\right)^{2}
\end{aligned}
$$

and in equilibrium, each trader has excess inventory

$$
\begin{aligned}
z_{t}^{i} & =z_{0}^{i}+Y^{i}\left(\hat{z}_{t}\right)+H_{t}^{i} \\
& =z_{0}^{i}+\frac{\sum_{j=1}^{n} \hat{z}_{t}^{j}}{n}-\hat{z}_{t}^{i}+H_{t}^{i} \\
& =z_{0}^{i}+\frac{\sum_{j=1}^{n}\left(z_{0}^{j}+H_{t}^{j}\right)}{n}-\left(z_{0}^{i}+H_{t}^{i}\right)+H_{t}^{i} \\
& =\bar{Z}_{t}
\end{aligned}
$$

almost everywhere. We have thus shown that the continuously run mechanisms achieve the first-best allocation while remaining budget balanced.

We now show that participation in this mechanism is not individually rational. Note that 
at the equilibrium strategy, trader $i$ 's expected payoff is

$$
\mathbb{E}\left[\int_{0}^{\infty} e^{-r t}\left(r v \bar{Z}_{t}+\tilde{T}_{\kappa}^{i}\left(\hat{z}_{t}\right)-\gamma\left(\bar{Z}_{t}\right)^{2}\right) d t\right]
$$

where, since $\sum_{j=1}^{n} \hat{z}_{t}^{j}=Z_{t}$, we have

$$
\begin{aligned}
\mathbb{E}\left[\int_{0}^{\infty} e^{-r t} \tilde{T}_{\kappa}^{i}\left(\hat{z}_{t}\right) d t\right]=\mathbb{E} & {\left[e^{-r t}\left(\kappa_{1} \hat{z}_{t}^{i}+\kappa_{0}\left(n \kappa_{2}+Z_{t}\right)^{2}+\kappa_{1} \kappa_{2}+\frac{\kappa_{1}^{2}}{4 \kappa_{0} n^{2}}\right) d t\right] } \\
=\mathbb{E} & {\left[\int_{0}^{\infty} e^{-r t} \kappa_{1}\left(z_{0}^{i}+H_{t}^{i}\right) d t+\int_{0}^{\infty} e^{-r t} \kappa_{0}\left(n \kappa_{2}+Z_{t}\right)^{2} d t\right] } \\
& +\frac{1}{r}\left[\kappa_{1} \kappa_{2}+\frac{\kappa_{1}^{2}}{4 \kappa_{0} n^{2}}\right] .
\end{aligned}
$$

Because $H^{i}$ is a martingale,

$$
\mathbb{E}\left[\int_{0}^{\infty} e^{-r t} \tilde{T}_{\kappa}^{i}\left(\hat{z}_{t}\right) d t\right]=\frac{\kappa_{1} z_{0}^{i}}{r}+\frac{2 \kappa_{0} n \kappa_{2} Z_{0}}{r}+\mathbb{E}\left[\int_{0}^{\infty} e^{-r t} \kappa_{0} Z_{t}^{2} d t\right]+\frac{1}{r}\left[\kappa_{1} \kappa_{2}+\frac{\kappa_{1}^{2}}{4 \kappa_{0} n^{2}}\right] .
$$

Thus the expected total profit of trader $i$ is

$$
\frac{\kappa_{1} z_{0}^{i}}{r}+Z_{0} \iota_{0}+\frac{n^{2} \kappa_{0}-\gamma}{r n^{2}} Z_{0}^{2}+\iota_{1}
$$

for some constants $\iota_{0}, \iota_{1}$. If trader $i$ could completely exit the mechanism, the associated expected payoff would be

$$
\mathbb{E}\left[z_{\mathcal{T}}^{i} \pi-\int_{0}^{\mathcal{T}} \gamma\left(z_{t}^{i}\right)^{2} d t\right]=v z_{0}^{i}-\frac{\gamma}{r}\left(z_{0}^{i}\right)^{2}+\iota_{2}
$$

for a constant $\iota_{2}$. From this, trader $i$ strictly prefers not to participate in the mechanism whenever $\left(z_{0}^{i}, Z_{0}\right)$ is in a specific subset of $\mathbb{R} \times \mathbb{R}^{n}$ with strictly positive Lebesgue measure. 